\title{
STOCHASTIC AIRY SEMIGROUP THROUGH TRIDIAGONAL MATRICES
}

\author{
BY VADIM GORIN ${ }^{*, \dagger, 1}$ AND MYKHAYLO SHKOLNIKOV $\$, 2$ \\ Massachusetts Institute of Technology*, Institute for Information Transmission \\ Problems of Russian Academy of Sciences ${ }^{\dagger}$ and Princeton University ${ }^{\dagger}$
}

We determine the operator limit for large powers of random symmetric tridiagonal matrices as the size of the matrix grows. The result provides a novel expression in terms of functionals of Brownian motions for the Laplace transform of the Airy $\beta$ process, which describes the largest eigenvalues in the $\beta$ ensembles of random matrix theory. Another consequence is a Feynman-Kac formula for the stochastic Airy operator of Edelman-Sutton and Ramirez-Rider-Virag.

As a side result, we find that the difference between the area underneath a standard Brownian excursion and one half of the integral of its squared local times is a Gaussian random variable.

\section{CONTENTS}

1. Introduction . . . . . . . . . . . . . . . . . . . . . . . . . . 2288

2. Setup and results . . . . . . . . . . . . . . . . . . . . . . . . . . . . 2289

3. Combinatorics of high powers of symmetric tridiagonal matrices . . . . . . . . . . . . 2297

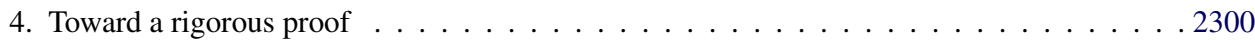

4.1. Convergence of random walk bridges and their local times . . . . . . . . . . 2300



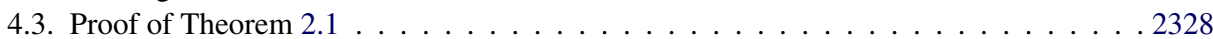

5. Properties of the stochastic Airy semigroup I . . . . . . . . . . . . . . . 2330

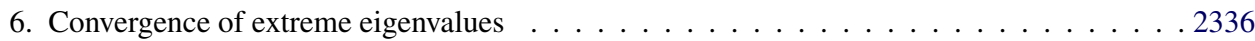

7. Properties of the stochastic Airy semigroup II . . . . . . . . . . . . . . . . . 2339

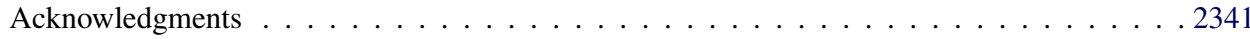

Supplementary Material . . . . . . . . . . . . . . . . . . . . 2341

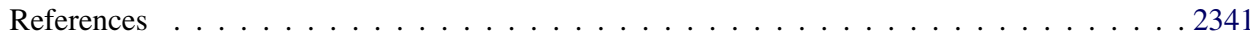

Received June 2016; revised August 2017.

${ }^{1}$ Supported in part by NSF Grants DMS-1407562, DMS-1664619 and by the Sloan Research Fellowship.

${ }^{2}$ Supported in part by NSF Grant DMS- 1506290 .

MSC2010 subject classifications. Primary 60B20, 60H25; secondary 47D08, 60G55, 60J55.

Key words and phrases. Airy point process, Brownian bridge, Brownian excursion, DumitriuEdelman model, Feynman-Kac formula, Gaussian beta ensemble, intersection local time, moment method, path transformation, quantile transform, random matrix soft edge, random walk bridge, stochastic Airy operator, strong invariance principle, trace formula, Vervaat transform. 
1. Introduction. This article is about spectral properties of random matrices, and we refer to $[1,2,32,53]$ for modern general reviews. In random matrix ensembles, one distinguishes a parameter $\beta$, which is typically equal to 1,2 or 4 in full Hermitian matrix models (such as Wigner or Wishart ensembles) and corresponds to real, complex or quaternion matrix elements. More generally, $\beta$ can be taken to be an arbitrary positive number, in relation with Coulomb log-gases, the Calogero-Sutherland quantum many-body system, random tridiagonal matrices, Heckman-Opdam and Macdonald processes; see, for example, [1], Chapter 20 "Beta Ensembles", [12, 25] for the details.

Here, we concentrate on edge limits of random matrix ensembles describing the asymptotic behavior of the largest eigenvalues (and the corresponding eigenvectors). At $\beta=2$, that is, for complex Hermitian matrices, there are many deep results in this direction. In particular, the properly centered and rescaled largest eigenvalue converges to the Tracy-Widom law $F_{2}$ [63], the point process describing all largest eigenvalues converges to the Airy point process, which is a part of 2D Airy line ensemble $[19,55]$ (the latter can be obtained by considering the largest eigenvalues of corners of random matrices). All these results are very robust and have been proved rigorously in great generality; see, for example, $[5,17$, $22,44,52,54,60,61]$. Furthermore, the universality of these objects extends far beyond random matrix theory; see, for example, [14, 15, 18, 39] and references therein. Several descriptions of the limiting objects are known: tractable expressions of their correlation functions (see, e.g., [55, 63]) and Laplace transforms (see, e.g., [51, 59, 61]), and a conjectural description through the so-called Brownian Gibbs property (see [19], Section 3.2).

While for $\beta=1$, there are still many results parallel to the $\beta=2$ case, there is much less understanding for general values of $\beta>0$. For the general $\beta$ analogues of $F_{2}$ and the Airy point process, the only known identification is via the spectrum of the stochastic Airy operator [30, 56], and no analytic formulas for correlation functions or Laplace transforms are known. Moreover, even the existence of the Airy line ensemble for general $\beta$ has not been established.

From the analytic point of view, the main difficulty for general $\beta$ is that the determinantal/Pfaffian formulas for the correlation functions available for $\beta=1,2,4$ are not known to extend to other values of $\beta$. A recent alternative approach producing explicit formulas through Macdonald processes [10, 11] does work for $\beta$ ensembles (see $[12,13]$ ), but the edge limits are not yet accessible through these techniques.

Another approach, which has proved to be very successful for $\beta=1,2,4$, is the moments method. In the present article, we prove that the latter approach can be adapted to the study of the edge limits of general $\beta$ ensembles. This leads to several outcomes. First, we prove that the Laplace transform of the point process of the rescaled largest eigenvalues in the Gaussian $\beta$ ensemble (and more general random matrices) converges to the Laplace transform of the Airy $\beta$ point process and establish a novel formula for the latter in terms of a functional of Brownian 
motion. This is closely related to our second result: the identification and proof of a Feynman-Kac formula for the stochastic Airy semigroup, the semigroup associated with the stochastic Airy operator.

It is known that Laplace transforms can be used to study various properties of the underlying point processes. For instance, by integrating one should be able to access linear statistics of the Airy ${ }_{\beta}$ point process (in addition to their intrinsic interest, the latter are also important for the study of rigidity properties; cf. [33]). On the other hand, by sending the parameter of the Laplace transform to infinity, one should be able to find the Tracy-Widom laws $F_{\beta}$. We postpone the discussion of these possible applications to future papers. Instead, we present a rather unexpected consequence: comparing our results with the literature for $\beta=2$ we find a novel identity involving the Brownian excursion area and the local times of the same excursion.

From the technical point of view, our main result is the computation of the asymptotics of matrix elements of large powers of random tridiagonal matrices. More precisely, for a matrix of size $N \times N$ we deal with powers of the order $N^{2 / 3}$. In the case when the powers do not grow or grow slower than $N^{2 / 3}$, such asymptotics has been previously analyzed in $[27,28,68]$, but for the analysis of the fast growing powers (directly related to the edge asymptotics of $\beta$ ensembles) many new ideas are necessary. In particular, in our proofs we heavily rely on strong invariance principles, that is, statements about the convergence of the trajectories of (conditioned) random walks to those of Brownian motions (or bridges) with a very precise control of errors, as in $[4,43,46]$. In addition, we use path transformations linking discrete local times of random walks to time-changed versions of the same random walk; see [3], and also [7, 21, 37] for the continuous analogues.

We proceed to a detailed exposition of our results.

Notation. In what follows, $C$ stands for a positive constant whose exact value is not important for us and might change from line to line.

2. Setup and results. Given two sequences of independent random variables $\mathfrak{a}(m), m \in \mathbb{N}$ and $\mathfrak{b}(m), m \in \mathbb{N}$, we define for each $N \in \mathbb{N}$ the $N \times N$ symmetric tridiagonal matrix $M_{N}=\left(M_{N}[m, n]\right)_{m, n=1}^{N}$ by setting $M_{N}[m, m]=\mathfrak{a}(m), m=$ $1,2, \ldots, N$ and $M[m, m+1]=\mathfrak{b}(m), m=1,2, \ldots, N-1$ :

$$
M_{N}=\left(\begin{array}{ccccc}
\mathfrak{a}(1) & \mathfrak{b}(1) & 0 & \ldots & 0 \\
\mathfrak{b}(1) & \mathfrak{a}(2) & \mathfrak{b}(2) & \ddots & \vdots \\
0 & \mathfrak{b}(2) & \mathfrak{a}(3) & \ddots & 0 \\
\vdots & \ddots & \ddots & \ddots & \mathfrak{b}(N-1) \\
0 & \ldots & 0 & \mathfrak{b}(N-1) & \mathfrak{a}(N)
\end{array}\right)
$$

In this paper, we study $M_{N}$ in the asymptotic regime $N \rightarrow \infty .^{3}$ 
The case when, for a fixed $\beta>0$, all $\mathfrak{a}(m), m \in \mathbb{N}$, have the normal distribution $N(0,2 / \beta)$, and the $\mathfrak{b}(m), m \in \mathbb{N}$, are $\beta^{-1 / 2}$ multiples of $\chi$-distributed random variables with parameters $\beta m, m \in \mathbb{N}$ is of particular interest. Here, the density of the $\chi$ distribution with parameter $a$ on $\mathbb{R}_{\geq 0}$ is

$$
\frac{2^{1-k / 2}}{\Gamma(a / 2)} x^{a-1} e^{-x^{2} / 2}, \quad x>0 .
$$

In this situation, the joint density of the $N$ eigenvalues $\lambda_{1} \leq \lambda_{2} \leq \cdots \leq \lambda_{N}$ of $M_{N}$ is proportional to

$$
\prod_{i<j}\left(\lambda_{j}-\lambda_{i}\right)^{\beta} \prod_{i=1}^{N} e^{-\frac{\beta}{4} \lambda_{i}^{2}},
$$

and the corresponding joint distribution is usually referred to as the Gaussian $\beta$ ensemble; see, for example, [26]. More generally, we work with arbitrary sequences of independent random variables $\mathfrak{a}(m), m \in \mathbb{N}$, and $\mathfrak{b}(m), m \in \mathbb{N}$, satisfying the following assumption.

Assumption 2.1. The sequences $\mathfrak{a}(m), m \in \mathbb{N}$ and $\mathfrak{b}(m), m \in \mathbb{N}$, of independent random variables satisfy, with the notation

$$
\mathfrak{b}(m)=\sqrt{m}+\xi(m), \quad m \in \mathbb{N},
$$

(a) as $m \rightarrow \infty,|\mathbb{E}[\mathfrak{a}(m)]|=o\left(m^{-1 / 3}\right),|\mathbb{E}[\xi(m)]|=o\left(m^{-1 / 3}\right)$;

(b) there exist nonnegative constants $s_{\mathfrak{a}}, s_{\xi}$ such that $\frac{s_{a}^{2}}{4}+s_{\xi}^{2}=\frac{1}{\beta}$ and, as $m \rightarrow$ $\infty, \mathbb{E}\left[\mathfrak{a}(m)^{2}\right]=s_{\mathfrak{a}}^{2}+o(1), \mathbb{E}\left[\xi(m)^{2}\right]=s_{\xi}^{2}+o(1) ;$

(c) there exist constants $C>0$ and $0<\gamma<2 / 3$ such that

$$
\mathbb{E}\left[|\mathfrak{a}(m)|^{\ell}\right] \leq C^{\ell} \ell^{\gamma \ell} \quad \text { and } \quad \mathbb{E}\left[|\xi(m)|^{\ell}\right] \leq C^{\ell} \ell^{\gamma \ell} \quad \text { for all } m, \ell \in \mathbb{N} \text {. }
$$

In particular, we have the following simple lemma.

LEMMA 2.1. If all $\mathfrak{a}(m), m \in \mathbb{N}$, are $N(0,2 / \beta)$-distributed and the $\sqrt{\beta} \mathfrak{b}(m)$, $m \in \mathbb{N}$, are $\chi$-distributed with parameters $\beta m, m \in \mathbb{N}$, respectively, then Assumption 2.1 holds with $s_{\mathfrak{a}} / 2=s_{\xi}=\frac{1}{\sqrt{2 \beta}}$.

PROOF. The result is immediate for $\mathfrak{a}(m), m \in \mathbb{N}$. For $\xi(m), m \in \mathbb{N}$ it follows from known tail estimates for $\chi$ random variables; see, for example, [45], Section 4.1, Lemma 1.

We start the study of the $N \rightarrow \infty$ limit of $M_{N}$ by recalling the semicircle law. For each $N \in \mathbb{N}$, let $\lambda_{N}^{1} \geq \lambda_{N}^{2} \geq \cdots \geq \lambda_{N}^{N}$ denote the ordered eigenvalues of $M_{N}$.

\footnotetext{
${ }^{3}$ All our arguments extend to the case when the entries $\mathfrak{a}(m), m=1,2, \ldots, N$ and $\mathfrak{b}(m), m=$ $1,2, \ldots, N-1$ vary with $N$. However, to keep the notation reasonable we have decided to work in the less general setup of no dependence on $N$.
} 
Consider the random probability measure

$$
\rho_{N}=\frac{1}{N} \sum_{i=1}^{N} \delta_{\lambda_{N}^{i} / \sqrt{N}}
$$

Proposition 2.1. Under Assumption 2.1, as $N \rightarrow \infty$, the random measure $\rho_{N}$ converge weakly, in probability, to the deterministic measure $\mu$ with density

$$
\frac{1}{2 \pi} \sqrt{4-x^{2}}, \quad-2<x<2 .
$$

For the Gaussian $\beta$ ensemble, Proposition 2.1 is well known and can be proven in several ways; cf. $[2,25]$. For the sake of completeness, we provide a proof of our more general statement in the Supplementary Material [34], Supplement A.

Proposition 2.1 gives the leading order asymptotics of the normalized spectral measure $\rho_{N}$. Refinements of this statement are available in at least three different directions. The first one studies the higher order asymptotics of $\rho_{N}$ in the same coordinates, that is, the fluctuations of $\rho_{N}$ around the semicircle distribution $\mu$. This is referred to as global asymptotics. In this direction, we prove in the Appendix [34], Supplement A a central limit theorem (CLT) for the joint fluctuations of multiple corners of $M_{N}$. Note that the Gaussian nature of the fluctuations (and the corresponding covariance structure) for a single matrix is well known; cf. $[2,25,38]$.

The second refinement is the study of the local asymptotics of the eigenvalues in the bulk of the spectrum. A typical question in this direction is the asymptotic distribution of the rescaled spacing $\sqrt{N}\left(\lambda_{N}^{\lfloor N / 2\rfloor}-\lambda_{N}^{\lfloor N / 2\rfloor+1}\right)$. We do not address this limiting regime in the present paper and instead refer to [64] for results of this type for random tridiagonal matrices.

The third refinement is the investigation of the asymptotics of the extreme eigenvalues of $M_{N}$ and the corresponding eigenvectors, which is known as edge asymptotics. In this direction, it is proven in $[44,56]$ (see also $[29,30,62]$ for earlier developments) that the random variable $N^{1 / 6}\left(\lambda_{N}^{i}-2 \sqrt{N}\right)$ converges in distribution for every fixed $i \in \mathbb{N}$. The limit of the corresponding eigenvector is also studied therein. Our main results are closely related to this work.

Let us now present the main results of this paper. Take a probability space which supports a standard Brownian motion $W$, and consider for each $T>0$ the following (random) kernel on $\mathbb{R}_{\geq 0} \times \mathbb{R}_{\geq 0}$ :

$$
\begin{aligned}
K(x, y ; T)= & \frac{1}{\sqrt{2 \pi T}} \exp \left(-\frac{(x-y)^{2}}{2 T}\right) \\
& \cdot \mathbb{E}_{B^{x, y}}\left[\mathbf { 1 } _ { \{ \forall t : B ^ { x , y } ( t ) \geq 0 \} } \operatorname { e x p } \left(-\frac{1}{2} \int_{0}^{T} B^{x, y}(t) \mathrm{d} t\right.\right. \\
& \left.\left.+\frac{1}{\sqrt{\beta}} \int_{0}^{\infty} L_{a}\left(B^{x, y}\right) \mathrm{d} W(a)\right)\right] .
\end{aligned}
$$


Here, $B^{x, y}$ is a standard Brownian bridge starting at $x$ at time 0 and ending at $y$ at time $T$ which is independent of $W$; the $L_{a}\left(B^{x, y}\right)$ are the local times accumulated by $B^{x, y}$ at level $a$ on $[0, T]$; and the expectation $\mathbb{E}_{B^{x, y}}$ is taken only with respect to $B^{x, y}$. We define $\mathcal{U}(T), T>0$, as the integral operators on $\mathbb{R}_{\geq 0}$ with kernels $K(x, y ; T), T>0$, respectively. In order to be able to make statements about $m u l$ tiple operators $\mathcal{U}(T)$, we use the same path of $W$ in (2.4) and define the stochastic integral with respect to $W$ therein according to the almost sure procedure described in [41]. For notational convenience, we let $\mathcal{U}(0)$ be the identity operator.

PROPOSITION 2.2. For each $T>0, \mathcal{U}(T)$ is almost surely a symmetric nonnegative trace class operator on $L^{2}\left(\mathbb{R}_{\geq 0}\right)$ satisfying the trace formula

$$
\operatorname{Trace}(\mathcal{U}(T))=\int_{\mathbb{R}_{\geq 0}} K(x, x ; T) \mathrm{d} x .
$$

Proposition 2.3. Operators $\mathcal{U}(T), T \geq 0$, have the almost sure semigroup property: for any $T_{1}, T_{2} \geq 0$, it holds $\mathcal{U}\left(T_{1}\right) \mathcal{U}\left(T_{2}\right)=\mathcal{U}\left(T_{1}+T_{2}\right)$ with probability one.

PROPOSITION 2.4. The semigroup $\mathcal{U}(T), T \geq 0$ is $L^{2}$-strongly continuous, that is, for any $T \geq 0$ and $f \in L^{2}\left(\mathbb{R}_{\geq 0}\right)$, it holds

$$
\lim _{t \rightarrow T} \mathbb{E}\left[\|\mathcal{U}(T) f-\mathcal{U}(t) f\|^{2}\right]=0
$$

PROPOSITION 2.5. There exists an orthonormal basis of random vectors $\mathbf{v}^{1}, \mathbf{v}^{2}, \ldots \in L^{2}\left(\mathbb{R}_{\geq 0}\right)$ and random variables $\eta^{1} \geq \eta^{2} \geq \cdots$ defined on the same probability space as $\mathcal{U}(T), T>0$, such that, for each $T>0$, the spectrum of $\mathcal{U}(T)$ [as an operator on $\left.L^{2}\left(\mathbb{R}_{\geq 0}\right)\right]$ consists of eigenvalues $\exp \left(T \eta^{i} / 2\right), i \in \mathbb{N}$ corresponding to the eigenvectors $\mathbf{v}^{i}, i \in \mathbb{N}$, respectively.

The proofs of Propositions 2.2, 2.3 and 2.4 are given in Section 5, and Proposition 2.5 is established in Section 7.

Our interest in the operators $\mathcal{U}(T), T>0$, is based on their appearance in the $N \rightarrow \infty$ edge limit of the matrix $M_{N}$ and its submatrices. More specifically, let $\mathcal{S}$ denote the set of all locally integrable functions $f$ on $\mathbb{R}_{\geq 0}$ which grow subexponentially fast at infinity [i.e., for which there exists a $\delta>0$ such that $f(x)=O\left(\exp \left(x^{1-\delta}\right)\right)$ as $\left.x \rightarrow \infty\right]$. Further, for any $N \in \mathbb{N}$ and $f \in \mathcal{S}$, write $\pi_{N} f$ for the vector in $\mathbb{R}^{N}$ with components $N^{1 / 6} \int_{N^{-1 / 3}(N-i)}^{N^{-1 / 3}(N-i+1)} f(x) \mathrm{d} x$, $i=1,2, \ldots, N$ and $\left(\pi_{N} f\right)^{\prime}$ for its transpose. In addition, define the $N \times N$ matrix

$$
\mathcal{M}(T, N)=\frac{1}{2}\left(\left(\frac{M_{N}}{2 \sqrt{N}}\right)^{\left\lfloor T N^{2 / 3}\right\rfloor}+\left(\frac{M_{N}}{2 \sqrt{N}}\right)^{\left\lfloor T N^{2 / 3}\right\rfloor-1}\right) .
$$


THEOREM 2.1. Under the Assumption 2.1, we have

$$
\lim _{N \rightarrow \infty} \mathcal{M}(T, N)=\mathcal{U}(T), \quad T \geq 0,
$$

in the following senses:

(a) Weak convergence: For any $f, g \in \mathcal{S}$ and $T \geq 0$, we have

$$
\lim _{N \rightarrow \infty}\left(\pi_{N} f\right)^{\prime} \mathcal{M}(T, N)\left(\pi_{N} g\right)=\int_{\mathbb{R}_{\geq 0}}\left(\mathcal{U}_{A}(T) f\right)(x) g(x) \mathrm{d} x
$$

in distribution and in the sense of moments.

(b) Convergence of traces: For any $T \geq 0$, we have

$$
\lim _{N \rightarrow \infty} \operatorname{Trace}(\mathcal{M}(T, N))=\operatorname{Trace}(\mathcal{U}(T))
$$

in distribution and in the sense of moments.

(c) The convergences in parts (a) and (b) also hold jointly for any finite collection of $T$ 's, $f$ 's and $g$ 's.

The convergence in distribution in part (c) is joint with the following limit in distribution with respect to the Skorokhod topology:

$$
W(a)=\sqrt{\beta} \lim _{N \rightarrow \infty} N^{-1 / 6} \sum_{n=N-\left\lfloor N^{1 / 3} a\right\rfloor}^{N}\left(\xi(n)+\frac{\mathfrak{a}(n)}{2}\right), \quad a \geq 0,
$$

where $W$ is the Brownian motion from the definition of $\mathcal{U}(T)$.

The proof of Theorem 2.1 is given in Section 4.3.

REMARK 2.2. We recall that, for deterministic operators, weak convergence together with the convergence of their traces imply other stronger forms of convergence, in particular, the convergence in the trace-class norm; see, for example, [58], Section 2. Yet, when we speak about the convergence of finite-dimensional distributions of random operators, sticking to the statements (a) and (b) seems quite natural.

REMARK 2.3. Fix a (possibly unbounded) interval $\mathcal{A} \subset \mathbb{R}_{\geq 0}$, consider for each $T>0$ the following kernel on $\mathbb{R}_{\geq 0} \times \mathbb{R}_{\geq 0}$ :

$$
\begin{aligned}
K_{\mathcal{A}}(x, y ; T)= & \frac{1}{\sqrt{2 \pi T}} \exp \left(-\frac{(x-y)^{2}}{2 T}\right) \\
& \cdot \mathbb{E}_{B^{x, y}}\left[\mathbf { 1 } _ { \{ \forall t : B ^ { x , y } ( t ) \in \mathcal { A } \} } \operatorname { e x p } \left(-\frac{1}{2} \int_{0}^{T} B^{x, y}(t) \mathrm{d} t\right.\right. \\
& \left.\left.+\frac{1}{\sqrt{\beta}} \int_{0}^{\infty} L_{a}\left(B^{x, y}\right) \mathrm{d} W(a)\right)\right]
\end{aligned}
$$


and define $\mathcal{U}_{\mathcal{A}}(T), T>0$, as the integral operators on $\mathbb{R}_{\geq 0}$ with kernels $K_{\mathcal{A}}(x, y ; T), T>0$, respectively. Let $M_{N ; \mathcal{A}}$ be the restriction of $M_{N}$ onto $\mathcal{A}$, so that the $(i, j)$ th entry of $M_{N, \mathcal{A}}$ is equal to that of $M_{N}$ if $\frac{N-i+1 / 2}{N^{1 / 3}}, \frac{N-j+1 / 2}{N^{1 / 3}}$ belong to $\mathcal{A}$ and zero otherwise. Set

$$
\mathcal{M}(T, \mathcal{A}, N)=\frac{1}{2}\left(\left(\frac{M_{N ; \mathcal{A}}}{2 \sqrt{N}}\right)^{\left\lfloor T N^{2 / 3}\right\rfloor}+\left(\frac{M_{N ; \mathcal{A}}}{2 \sqrt{N}}\right)^{\left\lfloor T N^{2 / 3}\right\rfloor-1}\right) .
$$

Then Theorem 2.1 can be generalized (and the proof remains the same with very minor modifications) to the convergence

$$
\lim _{N \rightarrow \infty} \mathcal{M}(T, \mathcal{A}, N)=\mathcal{U}_{\mathcal{A}}(T), \quad T \geq 0,
$$

which also holds jointly for finitely many $\mathcal{A}$ 's. The same remark applies to Corollary 2.1 below.

The convergence of the traces $\operatorname{Trace}(\mathcal{M}(T, N))$ as $N \rightarrow \infty$ implies the convergence of the eigenvalues of $M_{N}$ in the same limit. Let $\lambda_{N}^{1} \geq \lambda_{N}^{2} \geq \cdots \geq \lambda_{N}^{N}$ denote the eigenvalues of the matrix $N^{1 / 6}\left(M_{N}-2 \sqrt{N}\right)$.

COROLlaRY 2.1. In the notation of Proposition 2.5, one has the convergence in distribution

$$
\sum_{i=1}^{N} e^{T \lambda_{N}^{i} / 2} \longrightarrow_{N \rightarrow \infty} \sum_{i=1}^{\infty} e^{T \eta^{i} / 2}=\operatorname{Trace}(\mathcal{U}(T))
$$

jointly for finitely many $T$ 's. Therefore, one also has

$$
\lambda_{N}^{i} \longrightarrow_{N \rightarrow \infty} \eta^{i}
$$

jointly for finitely many i's.

REMARK 2.4. If we replace $M_{N}$ by $-M_{N}$ in the definition of $\mathcal{M}(T, N)$, then limit theorems for traces, similar to Theorem 2.1(b) and Corollary 2.1, will hold for this new object (see Remark 4.5 for more details). The latter give the asymptotics of the smallest eigenvalues of $M_{N}$. Interestingly, while for the variance constants $s_{\mathfrak{a}}, s_{\xi}$ corresponding to the Gaussian $\beta$ ensemble, the limits of the largest and the smallest eigenvalues are independent, this is not true in general.

The proof of Corollary 2.1 is given in Section 6 .

Let us compare our results with the previous work on the subject. An alternative derivation of the edge limit theorem and another interpretation of the limits $\eta^{i}$ were given in [56]. There the authors make sense of the stochastic Airy operator $S A O_{\beta}$

$$
S A O_{\beta}=-\frac{d^{2}}{d a^{2}}+a+\frac{2}{\sqrt{\beta}} W^{\prime}(a)
$$


on $L^{2}\left(\mathbb{R}_{\geq 0}\right)$ with a Dirichlet boundary condition at zero by appropriately defining an orthnormal basis of its eigenfunctions and the corresponding eigenvalues $-\eta^{i}$, $i \in \mathbb{N}$ (see [56], Section 2, and also [9, 50]). In addition, they show that the leading eigenvalues of $M_{N}$ (and the corresponding eigenvectors) converge to the leading eigenvalues (eigenvectors) of $S A O_{\beta}$. Note that, since the white noise $W^{\prime}(a), a \geq 0$ is a generalized function, special care is required in defining the operator $S A O_{\beta}$.

COROLlaRY 2.2. For any $T \geq 0$, define $e^{-\frac{T}{2} S A O_{\beta}}$ as the unique operator on $L^{2}\left(\mathbb{R}_{\geq 0}\right)$ with the same orthonormal basis of eigenfunctions as $S A O_{\beta}$ and the corresponding eigenvalues $e^{T \eta^{1} / 2} \geq e^{T \eta^{2} / 2} \geq \cdots$. If one couples $e^{-\frac{T}{2} S A O_{\beta}}$ with $\mathcal{U}(T)$ by identifying the Brownian motions $W$ in their respective definitions, then for each $T \geq 0$, the operators $e^{-\frac{T}{2} S A O_{\beta}}$ and $\mathcal{U}(T)$ coincide with probability one.

The proof of Corollary 2.2 is given in Section 7. Proposition 2.3 and Corollary 2.2 lead to the name stochastic Airy semigroup for the operators $\mathcal{U}(T), T \geq 0$.

The relationship between $S A O_{\beta}$ and the operators $\mathcal{U}(T), T>0$, can be viewed as a variant of the Feynman-Kac formula for Schroedinger operators (see, e.g., [57], Section 6, equation (6.6), for the case when the potential is a deterministic function). However, since in the case of $S A O_{\beta}$ the potential is given by the generalized function $a+\frac{2}{\sqrt{\beta}} W^{\prime}(a)$, such a result seems to be beyond the scope of the previous literature. A notable exception is the "zero temperature case" $\beta=\infty$ which falls into the framework of the usual Feynman-Kac formula. In that case, a path transformation argument allows to recast the Feynman-Kac identity

$\operatorname{Trace}(\mathcal{U}(T))$

$$
=\frac{1}{\sqrt{2 \pi T}} \int_{0}^{\infty} \mathbb{E}_{B^{x, x}}\left[\mathbf{1}_{\left\{\forall t: B^{x, x}(t) \geq 0\right\}} \exp \left(-\frac{1}{2} \int_{0}^{T} B^{x, x}(t) \mathrm{d} t\right)\right] \mathrm{d} x
$$

for the trace of $\mathcal{U}(T)$ as

$$
\operatorname{Trace}(\mathcal{U}(T))=\sqrt{\frac{2}{\pi}} T^{-3 / 2} \mathbb{E}\left[\exp \left(-\frac{T^{3 / 2}}{2} \int_{0}^{1} \mathfrak{e}(t) \mathrm{d} t\right)\right],
$$

where $\mathfrak{e}$ is a standard Brownian excursion on the time interval $[0,1]$ (see the proof of Proposition 2.7 for the details). Since $S A O_{\infty}$ is the deterministic Airy operator, the latter formula is the well-known series representation for the Laplace transform of the Brownian excursion area $\int_{0}^{1} \mathfrak{e}(t) \mathrm{d} t$ (see, e.g., [36], Section 13).

Let us also remark that [9], Appendix A, transforms $S A O_{\beta}$ to a classical SturmLiouville operator. It would be interesting (but possibly challenging) to compare the Feynman-Kac semigroup for this operator with our $\mathcal{U}(T)$.

We now turn to the special value $\beta=2$, in which case the edge asymptotics is much better understood. In particular, there exist formulas for the moments of the limiting traces of the form (2.9). The first moment admits a particularly simple formula and is given (in our notation) by the following proposition from [51]. 
PROPOSITION 2.6 ([51], Section 2.6.1). Take $\beta=2$. Then, for all $T>0$,

$$
\mathbb{E}[\operatorname{Trace}(\mathcal{U}(T))]=\mathbb{E}\left[\sum_{i \geq 1} e^{T \eta^{i} / 2}\right]=\sqrt{\frac{2}{\pi}} T^{-3 / 2} e^{T^{3} / 96} .
$$

On the other hand, our expression for the kernel $K(x, y ; T)$ and a suitable path transformation allow to write the same trace in terms of a functional of a standard Brownian excursion $\mathfrak{e}$ on the time interval $[0,1]$.

Proposition 2.7. For all $T>0$,

$$
\begin{aligned}
\mathbb{E}[\operatorname{Trace}(\mathcal{U}(T))] & \\
= & \mathbb{E}\left[\sum_{i \geq 1} e^{T \eta^{i} / 2}\right] \\
& =\sqrt{\frac{2}{\pi}} T^{-3 / 2} \mathbb{E}\left[\exp \left(-\frac{T^{3 / 2}}{2} \int_{0}^{1} \mathfrak{e}(t) \mathrm{d} t+\frac{T^{3 / 2}}{2 \beta} \int_{0}^{\infty}\left(l_{y}\right)^{2} \mathrm{~d} y\right)\right],
\end{aligned}
$$

where $\mathfrak{e}$ is a standard Brownian excursion on the time interval $[0,1]$, and each $l_{y}$ is the total local time of $\mathfrak{e}$ at level $y$.

The proof of Proposition 2.7 is given in Section 7.

Comparing Propositions 2.6 and 2.7 one obtains the following corollary of independent interest.

COROLLARY 2.3. Let $\mathfrak{e}$ be a standard Brownian excursion on the time interval $[0,1]$ and, for each $y \geq 0$, let $l_{y}$ be the total local time of $\mathfrak{e}$ at level $y$. Then

$$
\int_{0}^{1} \mathfrak{e}(t) \mathrm{d} t-\frac{1}{2} \int_{0}^{\infty}\left(l_{y}\right)^{2} \mathrm{~d} y
$$

is a Gaussian random variable of mean 0 and variance $\frac{1}{12}$.

The proof of Corollary 2.3 is given in Section 7. To the best of our knowledge, Corollary 2.3 is new and did not appear previously in the path transformation literature. However, it has been established in that literature (see [7, 21, 37], Theorem 2.1) that the two terms in (2.12) have the same distribution. In particular, this implies that the expectation of the random variable in (2.12) indeed equals to 0 . After this work was finished and posted on the arXiv, an alternative derivation of Corollary 2.3 (not relying on the random matrix theory) was found in [35].

Proposition 2.7 also gives a partial explanation for the special role that the value $\beta=2$ plays. Indeed, expanding the last exponential function in (2.11) into a power 
series we get

$$
\begin{aligned}
& \mathbb{E}[\operatorname{Trace}(\mathcal{U}(T))] \\
& =\sqrt{\frac{2}{\pi}} T^{-3 / 2}-\frac{1}{2 \sqrt{2 \pi}} \mathbb{E}\left[\int_{0}^{1} \mathfrak{e}(t) \mathrm{d} t-\frac{1}{\beta} \int_{0}^{\infty}\left(l_{y}\right)^{2} \mathrm{~d} y\right] \\
& \quad+\frac{1}{4 \sqrt{2 \pi}} T^{3 / 2} \mathbb{E}\left[\left(\int_{0}^{1} \mathfrak{e}(t) \mathrm{d} t-\frac{1}{\beta} \int_{0}^{\infty}\left(l_{y}\right)^{2} \mathrm{~d} y\right)^{2}\right]+\cdots .
\end{aligned}
$$

In particular, $\beta=2$ is the only case in which the second term in the expansion (2.13) vanishes.

3. Combinatorics of high powers of symmetric tridiagonal matrices. In this section, we give a sketch of the proof of Theorem 2.1 and, in particular, explain how the Brownian bridges $B^{x, y}$ and the Brownian motion $W$ in the definition of the kernel $K(x, y ; T)$ arise in the study of high powers of the matrix $M_{N}$. The technical estimates required to justify the steps of this sketch are then presented in Section 4 below, culminating in the complete proof of the theorem in Section 4.3.

Our aim is to study the matrix elements and the trace of a high power of the matrix $M_{N}$. By definition,

$$
\left(M_{N}\right)^{k}\left[i, i^{\prime}\right]=\sum M_{N}\left[i_{0}, i_{1}\right] M_{N}\left[i_{1}, i_{2}\right] \cdots M_{N}\left[i_{k-2}, i_{k-1}\right] M_{N}\left[i_{k-1}, i_{k}\right]
$$

where the sum is taken over sequences of integers $i_{0}, i_{1}, \ldots, i_{k}$ in $\{1,2, \ldots, N\}$ such that $i_{0}=i, i_{k}=i^{\prime}$, and $\left|i_{j}-i_{j-1}\right| \leq 1$ for all $j=1,2, \ldots, k$. Hereby, the factors of the form $M_{N}[m, m+1]$ or $M_{N}[m, m-1]$ [given by $\left.\mathfrak{b}(m)=\sqrt{m}+\xi(m)\right]$ are given by $\sqrt{m}$ at the leading order in $m$, whereas factors of the form $M_{N}[m, m]$ [given by $\mathfrak{a}(m)$ ] are of order 1 in $m$.

We are interested in $\mathcal{M}(T, N)$ and take first $k=\left\lfloor T N^{2 / 3}\right\rfloor$. Throughout the argument we assume that $k$ is even, with the odd case being very similar. Let us consider the sequences in (3.1) without "horizontal" segments $i_{j-1}=i_{j}$. Note that we need to assume that $i-i^{\prime}$ is even, as otherwise the sum is empty. With the notation $a \wedge b$ for $\min (a, b)$, the corresponding part of the sum in (3.1) is

$$
(2 \sqrt{N})^{k} \cdot \frac{1}{2^{k}} \sum_{\substack{1 \leq i_{0}, i_{1}, \ldots, i_{k} \leq N \\\left|i_{j}-i_{j-1}\right|=1 \text { for all } j \\ i_{0}=i, i_{k}=i^{\prime}}} \prod_{l=1}^{k} \frac{\sqrt{i_{l} \wedge i_{l-1}}}{\sqrt{N}}\left(1+\frac{\xi\left(i_{l} \wedge i_{l-1}\right)}{\sqrt{i_{l} \wedge i_{l-1}}}\right) .
$$

The prefactor $(2 \sqrt{N})^{k}$ corresponds to the scaling under which the limiting spectral interval is $[-1,1]$. It is also precisely the normalization of $M_{N}$ used in the definition of $\mathcal{M}(T, N)$, and we need to identify the $N \rightarrow \infty$ limit of the rest of the expression in (3.2).

Write $i^{*}$ for $\min \left(i_{0}, i_{1}, \ldots, i_{k}\right)$. It is not hard to see that the contribution of the sequences with $\frac{N-i^{*}}{N^{1 / 3}} \rightarrow \infty$ to the sum in (3.2) becomes negligible in the limit, 
so that one can restrict the attention to sequences with $\lim \sup _{N \rightarrow \infty} \frac{N-i^{*}}{N^{1 / 3}}<\infty$. In particular, we choose $i, i^{\prime}$ such that

$$
x:=\lim _{N \rightarrow \infty} \frac{N-i}{N^{1 / 3}}<\infty, \quad y:=\lim _{N \rightarrow \infty} \frac{N-i^{\prime}}{N^{1 / 3}}<\infty .
$$

Note further that we are summing over the trajectories of a simple random walk bridge with $k$ steps connecting $i$ to $i^{\prime}$ (i.e., a simple random walk with $k$ steps conditioned on having the prescribed endpoints). Our aim is to prove that the normalized sum converges to an integral with respect to the law of the Brownian bridge connecting $x$ to $y$.

Each summand in (3.2) can be trivially rewritten as

$$
\exp \left(\frac{1}{2} \sum_{l=1}^{k} \log \left(1-\frac{N-i_{l} \wedge i_{l-1}}{N}\right)+\sum_{l=1}^{k} \log \left(1+\frac{\xi\left(i_{l} \wedge i_{l-1}\right)}{\sqrt{i_{l} \wedge i_{l-1}}}\right)\right)
$$

For terms with $\lim \sup _{N \rightarrow \infty} \frac{N-i^{*}}{N^{1 / 3}}<\infty$, the arguments of the logarithms in the first sum are close to 1 , and one can use the formula $\log (1+z) \approx z$ (here, $z$ is of the $\operatorname{order} N^{-2 / 3}$, and there are order $N^{2 / 3}$ summands). Similarly, for the logarithms in the second sum, consider the Taylor expansion

$$
\log \left(1+\frac{\xi\left(i_{l} \wedge i_{l-1}\right)}{\sqrt{i_{l} \wedge i_{l-1}}}\right)=\frac{\xi\left(i_{l} \wedge i_{l-1}\right)}{\sqrt{i_{l} \wedge i_{l-1}}}-\frac{1}{2} \frac{\xi\left(i_{l} \wedge i_{l-1}\right)^{2}}{i_{l} \wedge i_{l-1}}+\cdots
$$

and note that already the second term is of order $N^{-1}$ (in expectation). Since there are order $N^{2 / 3}$ summands, only the first term can contribute to the $N \rightarrow \infty$ limit. Consequently, in that limit the expression from (3.3) can be replaced by

$$
\exp \left(-\frac{1}{2 N} \sum_{l=1}^{k}\left(N-i_{l} \wedge i_{l+1}\right)+\sum_{l=1}^{k} \frac{\xi\left(i_{l} \wedge i_{l-1}\right)}{\sqrt{i_{l} \wedge i_{l-1}}}\right)
$$

At this stage, we observe that

$$
\sum_{l=1}^{k} \frac{\xi\left(i_{l} \wedge i_{l-1}\right)}{\sqrt{i_{l} \wedge i_{l-1}}}=\sum_{h=i^{*}}^{N} \frac{\xi(h)}{\sqrt{h}}\left|\left\{l: i_{l} \wedge i_{l+1}=h\right\}\right| .
$$

A typical trajectory of a simple random walk bridge with $k$ steps connecting $i$ to $i^{\prime}$ visits an order of $k^{1 / 2}$ sites, and the corresponding "occupation times" $\mid\{l$ : $\left.i_{l} \wedge i_{l-1}=h\right\} \mid$ are of the order $k^{1 / 2}$. Therefore, for every such trajectory, the sum on the right-hand side of (3.5) is a sum of independent random variables with means of orders $o\left(h^{-2 / 3} k^{1 / 2}\right)=o\left(N^{-1 / 3}\right)$ and variances of orders $O\left(h^{-1} k\right)=$ $O\left(N^{-1 / 3}\right)$. Since there are an order of $N^{1 / 3}$ summands, the limit of the sum is given by the central limit theorem. More specifically, the described random walk bridge converges in the limit $N \rightarrow \infty$ to a standard Brownian bridge on $[0, T]$ connecting $x$ to $y$, its occupation times normalized by $N^{1 / 3}$ converge to the local 
times of the Brownian bridge, and so the variance of the limiting centered Gaussian random variable comes out to $s_{\xi}^{2} \int_{0}^{\infty} L_{a}\left(B^{x, y}\right)^{2} \mathrm{~d} a$ [see Assumption 2.1(b) and (2.4) for the notation]. That random variable can be written more explicitly as

$$
s_{\xi} \int_{0}^{\infty} L_{a}\left(B^{x, y}\right) \mathrm{d} W_{\xi}(a),
$$

where the Brownian motion $s_{\xi} W_{\xi}(a)$ is the limit of $N^{-1 / 6} \sum_{h=N-\left\lfloor N^{1 / 3} a\right\rfloor}^{N} \xi(h)$.

Next, by a standard application of Stirling's formula we find that the number of random walk bridges of length $k=\left\lfloor T N^{2 / 3}\right\rfloor$ connecting $i$ to $i^{\prime}$ behaves asymptotically as $2^{k} N^{-1 / 3} \sqrt{\frac{2}{\pi T}} e^{-(x-y)^{2} /(2 T)}$. Since the expression in (3.2) can be viewed as a multiple of the expectation of a functional with respect to the law of such a random walk bridge, its asymptotic behavior is given by the same multiple of the corresponding functional of the Brownian bridge $B^{x, y}$ :

$$
\begin{aligned}
& (2 \sqrt{N})^{k} N^{-1 / 3} \sqrt{\frac{2}{\pi T}} e^{-(x-y)^{2} /(2 T)} \\
& \cdot \mathbb{E}_{B^{x, y}}\left[\mathbf{1}_{\left\{\forall t: B^{x, y}(t) \geq 0\right\}} \exp \left(-\frac{1}{2} \int_{0}^{T} B^{x, y}(t) \mathrm{d} t+s \xi \int_{0}^{\infty} L_{a}\left(B^{x, y}\right) \mathrm{d} W(a)\right)\right] .
\end{aligned}
$$

Next, we turn to the sequences in (3.1) which have horizontal segments. We still work with an even $k$ and write $2 n$ for the number of horizontal segments. The corresponding sequences can be thought of as follows: take a sequence of length $k-2 n$ with no horizontal segments and insert $2 n$ horizontal segments at arbitrary spots. To analyze the effect of such insertion, we start with the case $n=1$. The corresponding part of the sum in (3.1), normalized by $(2 \sqrt{N})^{k}$, is given by

$$
\begin{gathered}
\frac{1}{2^{k-2}} \sum_{\substack{1 \leq i_{0}, i_{1}, \ldots, i_{k-2} \leq N \\
\left|i_{j}-i_{j-1}\right|=1 \text { for all } j \\
i_{0}=i, i_{k-2}=i^{\prime}}} \prod_{l=1}^{k-2} \frac{\sqrt{i_{l} \wedge i_{l-1}}}{\sqrt{N}}\left(1+\frac{\xi\left(i_{l} \wedge i_{l-1}\right)}{\sqrt{i_{l} \wedge i_{l-1}}}\right) \\
\cdot\left(\frac{1}{(2 \sqrt{N})^{2}} \sum_{0 \leq j \leq l \leq k-2} \mathfrak{a}\left(i_{j}\right) \mathfrak{a}\left(i_{l}\right)\right) .
\end{gathered}
$$

The last factor can be written as the sum of $\frac{1}{2} \frac{1}{(2 \sqrt{N})^{2}}\left(\sum_{j=0}^{k-2} \mathfrak{a}\left(i_{j}\right)\right)^{2}$ and $\frac{1}{2} \frac{1}{(2 \sqrt{N})^{2}} \sum_{j=0}^{k-2} \mathfrak{a}\left(i_{j}\right)^{2}$. An analysis as for the left-hand side of (3.5) shows that the first term tends to $\frac{1}{2}$ times the square of a Gaussian random variable with mean 0 and variance $\frac{s_{\mathfrak{a}}^{2}}{4} \int_{0}^{\infty} L_{a}\left(B^{x, y}\right)^{2} \mathrm{~d} a$, which we write as

$$
\frac{s_{\mathfrak{a}}}{2} \int_{0}^{\infty} L_{a}\left(B^{x, y}\right) \mathrm{d} W_{\mathfrak{a}}(a)
$$

Here, the Brownian motion $s_{\mathfrak{a}} W_{\mathfrak{a}}(a)$ is the limit of $N^{-1 / 6} \sum_{h=N-\left\lfloor N^{1 / 3} a\right\rfloor}^{N} \mathfrak{a}(h)$. The second term is of order $O\left(N^{-1} k\right)=O\left(N^{-1 / 3}\right)$ (in expectation), and so negligible in the limit $N \rightarrow \infty$. 
Similarly, for any number $2 n$ of horizontal segments, their leading order contribution is a factor of

$$
\frac{1}{(2 n) !(2 \sqrt{N})^{2 n}}\left(\sum_{j=0}^{k-2 n} \mathfrak{a}\left(i_{j}\right)\right)^{2 n} \sim \frac{1}{(2 n) !}\left(\frac{s_{\mathfrak{a}}}{2} \int_{0}^{\infty} L_{a}\left(B^{x, y}\right) \mathrm{d} W_{\mathfrak{a}}(a)\right)^{2 n} .
$$

At this point, we add $\left(M_{N}\right)^{k-1}\left[i, i^{\prime}\right]$ [as in the definition of $\left.\mathcal{M}(T, N)\right]$ and recall that $k-1$ is odd. Treating $\left(M_{N}\right)^{k-1}\left[i, i^{\prime}\right]$ exactly in the same way as $\left(M_{N}\right)^{k}\left[i, i^{\prime}\right]$, only changing the even number of horizontal segments to an odd number $2 n-1$ of horizontal segments, we conclude that the leading order contributions of the latter combine to

$$
\begin{aligned}
\sum_{n=1}^{\infty} \frac{1}{(2 n-1) !}\left(\frac{s_{\mathfrak{a}}}{2} \int_{0}^{\infty} L_{a}\left(B^{x, y}\right) \mathrm{d} W_{\mathfrak{a}}(a)\right)^{2 n-1} \\
\quad+\sum_{n=0}^{\infty} \frac{1}{(2 n) !}\left(\frac{s_{\mathfrak{a}}}{2} \int_{0}^{\infty} L_{a}\left(B^{x, y}\right) \mathrm{d} W_{\mathfrak{a}}(a)\right)^{2 n} \\
=\exp \left(\frac{s_{\mathfrak{a}}}{2} \int_{0}^{\infty} L_{a}\left(B^{x, y}\right) \mathrm{d} W_{\mathfrak{a}}(a)\right) .
\end{aligned}
$$

Putting everything together, we obtain the asymptotics

$$
\begin{aligned}
& \frac{1}{2}\left(\left(\frac{M_{N}}{2 \sqrt{N}}\right)^{k}+\left(\frac{M_{N}}{2 \sqrt{N}}\right)^{k-1}\right)\left[i, i^{\prime}\right] \\
& \sim N^{-1 / 3} \sqrt{\frac{1}{2 \pi T}} e^{-\frac{(x-y)^{2}}{2 T}} \mathbb{E}_{B^{x, y}}\left[\mathbf { 1 } _ { \{ \forall t : B ^ { x , y } ( t ) \geq 0 \} } \operatorname { e x p } \left(-\frac{1}{2} \int_{0}^{T} B^{x, y}(t) \mathrm{d} t\right.\right. \\
& \left.\left.+\int_{0}^{\infty} L_{a}\left(B^{x, y}\right)\left(s_{\xi} \mathrm{d} W_{\xi}(a)+\frac{s_{\mathfrak{a}}}{2} \mathrm{~d} W_{\mathfrak{a}}(a)\right)\right)\right] .
\end{aligned}
$$

To complete the derivation of the kernel $K(x, y ; T)$, it remains to recall from Assumption 2.1 that $s_{\xi}^{2}+\frac{s_{\mathfrak{a}}^{2}}{4}=\frac{1}{\beta}$ and to set $W=\sqrt{\beta}\left(s_{\xi} W_{\xi}+\frac{s_{\mathfrak{a}}}{2} W_{\mathfrak{a}}\right)$. Theorem 2.1 now follows by summing (3.6) over the relevant indices $i, i^{\prime}$ and replacing the sums by the integrals they approximate. Finally, for odd $i-i^{\prime}$, one only needs to interchange the roles of even and odd powers of $M_{N}$.

\section{Toward a rigorous proof.}

4.1. Convergence of random walk bridges and their local times. In this subsection, we present the main technical ingredients needed to justify the arguments of Section 3.

We use the following set of notation. Let $x, y \in \mathbb{R}, N \in \mathbb{N}$, and $\tilde{T}>0$ be such that $\tilde{T} N^{2 / 3}$ is an integer with parity of $\left\lfloor N-N^{1 / 3} x\right\rfloor-\left\lfloor N-N^{1 / 3} y\right\rfloor$. We write

$$
X^{x, y ; N, \tilde{T}}:=\left(X^{x, y ; N, \tilde{T}}(0), X^{x, y ; N, \tilde{T}}\left(N^{-2 / 3}\right), \ldots, X^{x, y ; N, \tilde{T}}(\tilde{T})\right)
$$


for the simple random walk bridge connecting $\left\lfloor N-N^{1 / 3} x\right\rfloor$ to $\left\lfloor N-N^{1 / 3} y\right\rfloor$ in $\tilde{T} N^{2 / 3}$ steps of size \pm 1 . More specifically, the trajectory of $X^{x, y ; N, \tilde{T}}$ is chosen uniformly at random among all trajectories of the type described. We further define $X^{x, y ; N, \tilde{T}}(t)$ for all $t \in[0, \tilde{T}]$ by linear interpolation. Finally, for $h \in \mathbb{R}$, we introduce the (normalized) occupation times

$$
\begin{aligned}
& L_{h}\left(X^{x, y ; N, \tilde{T}}\right) \\
& \quad=N^{-1 / 3}\left|\left\{t \in[0, \tilde{T}]: X^{x, y ; N, \tilde{T}}(t)=N-N^{1 / 3} h\right\}\right|, \quad h \in \mathbb{R} .
\end{aligned}
$$

The next proposition provides a coupling of the quantities $L_{h}\left(X^{x, y ; N, \tilde{T}}\right), h \in \mathbb{R}$ with their continuous analogues, the local times of a Brownian bridge.

Proposition 4.1. Fix $x, y \in \mathbb{R}$. Let $T_{N}, N \in \mathbb{N}$ be a sequence of reals such that $\sup _{N}\left|T_{N}-T\right| N^{2 / 3}<\infty$ for some $T>0, T_{N} N^{2 / 3} \in \mathbb{N}$ for all $N$, and $T_{N} N^{2 / 3}$ has the same parity as $\left\lfloor N-N^{1 / 3} x\right\rfloor-\left\lfloor N-N^{1 / 3} y\right\rfloor$. Then there exists a probability space supporting a sequence of random walk bridges $X^{x, y ; N, T_{N}}, N \in \mathbb{N}, a$ standard Brownian bridge $B^{x, y}$ on $[0, T]$ connecting $x$ to $y$, and a real random variable $\mathcal{C}$ such that for all $N \in \mathbb{N}$ :

$$
\begin{gathered}
\sup _{h \in \mathbb{R}}\left|L_{h}\left(X^{x, y ; N, T_{N}}\right)-L_{h}\left(B^{x, y}\right)\right| \leq \mathcal{C} N^{-1 / 16}, \\
\sup _{0 \leq t \leq T_{N} \wedge T}\left|N^{-1 / 3}\left(N-X^{x, y ; N, T_{N}}(t)\right)-B^{x, y}(t)\right| \leq \mathcal{C} N^{-1 / 3} \log N .
\end{gathered}
$$

Strong invariance principles similar to Proposition 4.1 can be found in the literature; cf. [16], Remark 1.3, [20], Theorem 2.2.4, for the case of random walks and [43], Theorem 2, for the case of bridges of compensated Poisson processes. The proof of Proposition 4.1 is given in the Appendix [34], Supplement B, and is based on a coupling constructed in [46] and a lemma from [4].

Proposition 4.1 describes the typical behavior of the random walk bridges $X^{x, y ; N, T_{N}}, N \in \mathbb{N}$ and the associated occupation times $L_{h}\left(X^{x, y ; N, T_{N}}\right), h \in \mathbb{R}$, $N \in \mathbb{N}$. Next, we complement it by some estimates on the tail behavior of these quantities. To this end, for each $x, y \in \mathbb{R}, N \in \mathbb{N}$, and $T_{0}>0$, we write $\mathcal{T}\left(x, y ; N, T_{0}\right)$ for the set of $\tilde{T} \in\left[0, T_{0}\right)$ such that $\tilde{T} N^{2 / 3}$ is an integer of the same parity as $\left\lfloor N-N^{1 / 3} x\right\rfloor-\left\lfloor N-N^{1 / 3} y\right\rfloor$.

Proposition 4.2. For every $T_{0}>0$ and $\theta \in \mathbb{R}$, one has the uniform integrability estimate

$$
\sup _{\substack{N \in \mathbb{N} \\ \quad \sup _{\tilde{T}} \in \mathcal{T}\left(x, y ; N, T_{0}\right)}} \mathbb{E}\left[\exp \left(\theta N^{-2 / 3} \sum_{i=0}^{\tilde{T} N^{2 / 3}} N^{-1 / 3}\left(N-X^{x, y ; N, \tilde{T}}\left(i N^{-2 / 3}\right)\right)\right)\right]
$$

uniformly on compact sets in $x, y$. 
PROPOSITION 4.3. For every $T_{0}>0,1 \leq p<3$ and $\theta>0$, one has the uniform integrability estimate

$$
\sup _{N \in \mathbb{N}} \sup _{\tilde{T} \in \mathcal{T}\left(x, y ; N, T_{0}\right)} \mathbb{E}\left[\exp \left(\theta N^{-1 / 3} \sum_{h \in N^{-1 / 3} \mathbb{Z}} L_{h}\left(X^{x, y ; N, \tilde{T}}\right)^{p}\right)\right]<\infty
$$

uniformly on compact sets in $x, y$.

REMARK 4.1. The trivial estimate of $L_{h}\left(X^{x, y ; N, \tilde{T}}\right)$ by

$$
L_{N^{2 / 3}-N^{-1 / 3}\left\lceil N-N^{1 / 3} h\right\rceil}\left(X^{x, y ; N, \tilde{T}}\right)+L_{N^{2 / 3}-N^{-1 / 3}\left\lfloor N-N^{1 / 3} h\right\rfloor}\left(X^{x, y ; N, \tilde{T}}\right)
$$

for $h \in \mathbb{R}$ shows that (4.5) continues to hold if one replaces the summation over $h \in N^{-1 / 3} \mathbb{Z}$ by the summation over $h \in c+N^{-1 / 3} \mathbb{Z}$ for some $c \in \mathbb{R}$.

Proof OF Proposition 4.2. Define the random walk bridge

$$
\tilde{X}^{x, y ; N, \tilde{T}}(t):=N^{-1 / 3}\left(N-X^{x, y ; N, \tilde{T}}(t)\right), \quad t \in[0, \tilde{T}]
$$

and note that its endpoints $x_{N}, y_{N}$ satisfy $\left|x_{N}-x\right| \leq N^{-1 / 3},\left|y_{N}-y\right| \leq N^{-1 / 3}$. Moreover, due to the symmetry properties of random walk bridges, we may assume without loss of generality that $\theta>0$ and $x_{N} \leq y_{N}$. We also observe that the random variable inside the expectation in (4.4) can be bounded from above by $e^{\theta\left(T_{0}+N^{-2 / 3}\right) \tilde{M}(N, \tilde{T})}$ where $\tilde{M}(N, \tilde{T})$ is the maximum of $\tilde{X}^{x, y ; N, \tilde{T}}$, that is, $\tilde{M}(N, \tilde{T})=\max _{t \in[0, \tilde{T}]} \tilde{X}^{x, y ; N, \tilde{T}}(t)$. Since a bridge with a larger endpoint has a higher probability of going up at all sites (resulting in a monotone coupling between bridges with different endpoints), we may assume further that $x_{N} \in\left\{y_{N}-N^{-1 / 3}, y_{N}\right\}$, with the choice depending on the parity of $\tilde{T} N^{2 / 3}$. We focus on the case that $\tilde{T} N^{2 / 3}$ is even and $y_{N}=x_{N}$, and the other case can be dealt with similarly.

In order to upper bound the exponential moments of $\tilde{M}(N, \tilde{T})$, we view $\tilde{X}^{x, y ; N, \tilde{T}}$ as a simple symmetric random walk $\tilde{X}^{x ; N, \tilde{T}}$ with steps $\pm N^{-1 / 3}$, started at $x_{N}$ and conditioned to also end at $x_{N}$. The reflection principle for simple symmetric random walks gives for $i \in \mathbb{Z}, i N^{-1 / 3} \geq x_{N}$, and with the notation 


$$
\begin{aligned}
& \mathfrak{T}:=\tilde{T} N^{2 / 3} / 2, \mathfrak{x}:=N^{1 / 3} x_{N}, \\
& \mathbb{P}\left(\tilde{M}(N, \tilde{T})=i N^{-1 / 3}\right) \\
& =\frac{\mathbb{P}\left(\tilde{X}^{x ; N, \tilde{T}}(\tilde{T})=2 i N^{-1 / 3}-x_{N}\right)-\mathbb{P}\left(\tilde{X}^{x ; N, \tilde{T}}(\tilde{T})=2 N^{-1 / 3}(i+1)-x_{N}\right)}{\mathbb{P}\left(\tilde{X}^{x ; N, \tilde{T}}(\tilde{T})=x_{N}\right)} \\
& =\frac{\left(\begin{array}{c}
2 \mathfrak{T} \\
\mathfrak{T}+i-\mathfrak{x}
\end{array}\right)-\left(\begin{array}{c}
2 \mathfrak{T} \\
\mathfrak{T}+i+1-\mathfrak{x}
\end{array}\right)}{\left(\begin{array}{c}
2 \mathfrak{T} \\
\mathfrak{T}
\end{array}\right)} \\
& =\frac{\mathfrak{T} ! \mathfrak{T} !}{(\mathfrak{T}+i-\mathfrak{x}) !(\mathfrak{T}-i+\mathfrak{x}) !}-\frac{\mathfrak{T} ! \mathfrak{T} !}{(\mathfrak{T}+i-\mathfrak{x}+1) !(\mathfrak{T}-i+\mathfrak{x}-1) !} \\
& =\frac{\mathfrak{T} ! \mathfrak{T} !}{(\mathfrak{T}+i-\mathfrak{x}) !(\mathfrak{T}-i+\mathfrak{x}) !}\left(1-\frac{\mathfrak{T}-i+\mathfrak{x}}{\mathfrak{T}+i-\mathfrak{x}+1}\right) \\
& =\frac{\mathfrak{T} ! \mathfrak{T} !}{(\mathfrak{T}+i-\mathfrak{x}+1) !(\mathfrak{T}-i+\mathfrak{x}) !} \text {. }
\end{aligned}
$$

At this point, using standard estimates on the factorials based on the Stirling's formula for the last expression in (4.6), one sees that for each $0<p<2$ and $\theta>0$ :

$$
\sup _{N \in \mathbb{N}} \mathbb{E}\left[e^{\theta \tilde{M}(N, \tilde{T})^{p}}\right]<\infty
$$

Our proof of Proposition 4.3 relies on an identity in distribution from [3] relating the maximal occupation time of $X^{x, y ; N, \tilde{T}}$ to the range of $X^{x, y ; N, \tilde{T}}$. The latter builds on the concepts of quantile and Vervaat transforms from [3] and [66], respectively.

DEFINITION 4.1 (Quantile transform). For a sequence $i_{0}, i_{1}, \ldots, i_{k}$ with $\mid i_{l}-$ $i_{l-1} \mid=1$ for all $l$, let $\kappa$ be the unique permutation of $\{1,2, \ldots, k\}$ such that, for all $1 \leq l_{1}<l_{2} \leq k$, either $i_{\kappa\left(l_{1}\right)-1}<i_{\kappa\left(l_{2}\right)-1}$, or $i_{\kappa\left(l_{1}\right)-1}=i_{\kappa\left(l_{2}\right)-1}$ and $\kappa\left(l_{1}\right)<\kappa\left(l_{2}\right)$. Then the quantile transform $q_{0}, q_{1}, \ldots, q_{k}$ of $i_{0}, i_{1}, \ldots, i_{k}$ is defined by $q_{0}=0$,

$$
q_{l}=\sum_{l_{1}=1}^{l}\left(i_{\kappa\left(l_{1}\right)}-i_{\kappa\left(l_{1}\right)-1}\right), \quad l=1,2, \ldots, k .
$$

In words: the quantile transform $q_{0}, q_{1}, \ldots, q_{k}$ is obtained by starting at 0 and consecutively adding the increments of $i_{0}, i_{1}, \ldots, i_{k}$ in the nondecreasing order of the values they originate from in $i_{0}, i_{1}, \ldots, i_{k}$, with the increments originating from the same value in $i_{0}, i_{1}, \ldots, i_{k}$ being added in chronological order. We refer to [3], Figure 1, for an illustration.

Using the construction of Definition 4.1 for the random walk bridge $X^{x, y ; N, \tilde{T}}$, we find the permutation $\kappa$ of $\left\{1,2, \ldots, \tilde{T} N^{2 / 3}\right\}$ such that, for all $1 \leq l_{1}<l_{2} \leq$ 
$\tilde{T} N^{2 / 3}$, either $X^{x, y ; N, \tilde{T}}\left(\left(\kappa\left(l_{1}\right)-1\right) N^{-2 / 3}\right)<X^{x, y ; N, \tilde{T}}\left(\left(\kappa\left(l_{2}\right)-1\right) N^{-2 / 3}\right)$, or $X^{x, y ; N, \tilde{T}}\left(\left(\kappa\left(l_{1}\right)-1\right) N^{-2 / 3}\right)=X^{x, y ; N, \tilde{T}}\left(\left(\kappa\left(l_{2}\right)-1\right) N^{-2 / 3}\right)$ and $\kappa\left(l_{1}\right)<\kappa\left(l_{2}\right)$. The quantile transform $Q^{N, \tilde{T}}$ of $X^{x, y ; N, \tilde{T}}$ is then given by $Q^{N, \tilde{T}}(0)=0$,

$$
\begin{aligned}
Q^{N, \tilde{T}}\left(l N^{-2 / 3}\right)= & \sum_{l_{1}=1}^{l}\left(X^{x, y ; N, \tilde{T}}\left(\kappa\left(l_{1}\right) N^{-2 / 3}\right)\right. \\
& \left.-X^{x, y ; N, \tilde{T}}\left(\left(\kappa\left(l_{1}\right)-1\right) N^{-2 / 3}\right)\right), \\
& l=1,2, \ldots, \tilde{T} N^{2 / 3}
\end{aligned}
$$

DEFINITION 4.2 (Vervaat transform). For a sequence $i_{0}, i_{1}, \ldots, i_{k}$ with $\mid i_{l}-$ $i_{l-1} \mid=1$ for all $l$, let

$$
l^{*}=\min \left\{l_{1} \in\{1,2, \ldots, k\}: i_{l_{2}} \geq i_{l_{1}} \text { for all } l_{2}\right\}
$$

and write $\vartheta$ for the permutation of $\{1,2, \ldots, k\}$ mapping each element $l_{1}$ to $l_{1}+l^{*}$ $\bmod k$. Then the Vervaat transform $v_{0}, v_{1}, \ldots, v_{k}$ of $i_{0}, i_{1}, \ldots, i_{k}$ is defined by $v_{0}=0$,

$$
v_{l}=\sum_{l_{1}=1}^{l}\left(i_{\vartheta\left(l_{1}\right)}-i_{\vartheta\left(l_{1}\right)-1}\right), \quad l=1,2, \ldots, k .
$$

In words: the Vervaat transform $v_{0}, v_{1}, \ldots, v_{k}$ is obtained from $i_{0}, i_{1}, \ldots, i_{k}$ by splitting the path associated with $i_{0}, i_{1}, \ldots, i_{k}$ at its first global minimum, attaching the first part of the path to the endpoint of the second part of the path, and shifting the resulting path so that it starts at 0 . We refer to [3], Figure 17, for an illustration.

Executing the construction of Definition 4.2 for $X^{x, y ; N, \tilde{T}}$ we let

$$
\begin{aligned}
l^{*}= & \min \left\{l_{1} \in\left\{1,2, \ldots, \tilde{T} N^{2 / 3}\right\}:\right. \\
& \left.X^{x, y ; N, \tilde{T}}\left(l_{2} N^{-2 / 3}\right) \geq X^{x, y ; N, \tilde{T}}\left(l_{1} N^{-2 / 3}\right) \text { for all } l_{2}\right\}
\end{aligned}
$$

and write $\vartheta$ for the permutation of $\left\{1,2, \ldots, \tilde{T} N^{2 / 3}\right\}$ mapping each element $l_{1}$ to $l_{1}+l^{*} \bmod \tilde{T} N^{2 / 3}$. The Vervaat transform $V^{N, \tilde{T}}$ of $X^{x, y ; N, \tilde{T}}$ is then determined by $V^{N, \tilde{T}}(0)=0$,

$$
\begin{aligned}
V^{N, \tilde{T}}\left(l N^{-2 / 3}\right)= & \sum_{l_{1}=1}^{l}\left(X^{x, y ; N, \tilde{T}}\left(\vartheta\left(l_{1}\right) N^{-2 / 3}\right)\right. \\
& \left.-X^{x, y ; N, \tilde{T}}\left(\left(\vartheta\left(l_{1}\right)-1\right) N^{-2 / 3}\right)\right), \\
& l=1,2, \ldots, \tilde{T} N^{2 / 3} .
\end{aligned}
$$


Proof OF Proposition 4.3. For each $h \in N^{-1 / 3} \mathbb{Z}$, we write $u_{h}^{N, \tilde{T}}$ and $d_{h}^{N, \tilde{T}}$ for the numbers of up and down steps of $X^{x, y ; N, \tilde{T}}$ originating from $N-N^{1 / 3} h$, respectively, and define

$$
t_{h}^{N, \tilde{T}}=\sum_{N^{-1 / 3} \mathbb{Z} \ni h_{1}>h}\left(u_{h_{1}}^{N, \tilde{T}}+d_{h_{1}}^{N, \tilde{T}}\right) .
$$

Using these and the elementary inequality

$$
(a+b)^{c} \leq 2^{c}\left(a^{c}+b^{c}\right), \quad a, b, c \geq 0
$$

with $c=p-1$ we obtain

$$
\begin{aligned}
& \sum_{h \in N^{-1 / 3} \mathbb{Z}} L_{h}\left(X^{x, y ; N, \tilde{T}}\right)^{p} \\
& =N^{-p / 3} \sum_{h \in N^{-1 / 3} \mathbb{Z}}\left(u_{h}^{N, \tilde{T}}+d_{h}^{N, \tilde{T}}\right)^{p} \\
& \leq N^{-p / 3} 2^{p-1} \sum_{h \in N^{-1 / 3} \mathbb{Z}}\left(\left(u_{h}^{N, \tilde{T}}+d_{h}^{N, \tilde{T}}\right)\left(u_{h}^{N, \tilde{T}}\right)^{p-1}\right. \\
& \left.\quad+\left(u_{h}^{N, \tilde{T}}+d_{h}^{N, \tilde{T}}\right)\left(d_{h}^{N, \tilde{T}}\right)^{p-1}\right) .
\end{aligned}
$$

Next, we employ the following combinatorial identity (see [3], equation (5.3)):

$$
\begin{aligned}
Q^{N, \tilde{T}} & \left(t_{h-N^{-1 / 3}}^{N, \tilde{T}} N^{-2 / 3}\right) \\
= & u_{h}^{N, \tilde{T}}+\left(N-N^{1 / 3} h-\left\lfloor N-N^{1 / 3} x\right\rfloor\right)_{+} \\
& \quad-\left(N-N^{1 / 3} h-\left\lfloor N-N^{1 / 3} y\right\rfloor\right)_{+}, \quad h \in N^{-1 / 3} \mathbb{Z}
\end{aligned}
$$

(note that in [3] the height of the bridge is computed with respect to the minimum of the bridge rather than zero, but this is not important). The latter gives the bound

$$
u_{h}^{N, \tilde{T}} \leq Q^{N, \tilde{T}}\left(t_{h-N^{-1 / 3}}^{N, \tilde{T}} N^{-2 / 3}\right)+N^{1 / 3}|x-y|+1, \quad h \in N^{-1 / 3} \mathbb{Z} .
$$

Moreover, by the combinatorial constraints $\left|d_{h-N^{-1 / 3}}^{N, \tilde{T}}-u_{h}^{N, \tilde{T}}\right| \leq 1$ for all $h \in$ $N^{-1 / 3} \mathbb{Z}$, so

$$
d_{h}^{N, \tilde{T}} \leq Q^{N, \tilde{T}}\left(t_{h}^{N, \tilde{T}} N^{-2 / 3}\right)+N^{1 / 3}|x-y|+2, \quad h \in N^{-1 / 3} \mathbb{Z} .
$$


Combining (4.16) with $u_{h}^{N, \tilde{T}}+d_{h}^{N, \tilde{T}}=t_{h-N^{-1 / 3}}^{N, \tilde{T}}-t_{h}^{N, \tilde{T}}, h \in N^{-1 / 3} \mathbb{Z}$ and (4.17), (4.18) we find

$$
\begin{aligned}
\sum_{h \in N^{-1 / 3} \mathbb{Z}} & L_{h}\left(X^{x, y ; N, \tilde{T}}\right)^{p} \\
\leq & N^{-p / 3} 2^{p-1}\left(\sum _ { h \in N ^ { - 1 / 3 } \mathbb { Z } } ( t _ { h - N ^ { - 1 / 3 } } ^ { N , \tilde { T } } - t _ { h } ^ { N , \tilde { T } } ) \left(Q^{N, \tilde{T}}\left(t_{h-N^{-1 / 3}}^{N, \tilde{T}} N^{-2 / 3}\right)\right.\right. \\
& \left.+N^{1 / 3}|x-y|+1\right)^{p-1} \\
& +\sum_{h \in N^{-1 / 3} \mathbb{Z}}\left(t_{h-N^{-1 / 3}}^{N, \tilde{T}}-t_{h}^{N, \tilde{T}}\right)\left(Q^{N, \tilde{T}}\left(t_{h}^{N, \tilde{T}} N^{-2 / 3}\right)\right. \\
& \left.\left.+N^{1 / 3}|x-y|+2\right)^{p-1}\right) .
\end{aligned}
$$

Estimating the values of $Q^{N, \tilde{T}}$ by $N^{1 / 3}$ times the maximal value $M(N, \tilde{T})$ taken by $N^{-1 / 3} Q^{N, \tilde{T}}$, noting $\sum_{h \in N^{-1 / 3} \mathbb{Z}}\left(t_{h-N^{-1 / 3}}^{N, \tilde{T}}-t_{h}^{N, \tilde{T}}\right)=\tilde{T} N^{2 / 3}$, and applying (4.15) with $c=p-1$ we get

$$
\begin{aligned}
N^{-1 / 3} & \sum_{h \in N^{-1 / 3} \mathbb{Z}} L_{h}\left(X^{x, y ; N, \tilde{T}}\right)^{p} \\
\leq & 2^{2 p-1} \tilde{T}\left(M(N, \tilde{T})^{p-1}+\left(|x-y|+2 N^{-1 / 3}\right)^{p-1}\right) .
\end{aligned}
$$

It remains to bound the exponential moments of the right-hand side of (4.19). To this end, we use the fact that the distribution of $M(N, \tilde{T})$ is the same as the distribution of the maximum of the normalized Vervaat transform $N^{-1 / 3} V^{N, \tilde{T}}$ (see [3], Corollary 7.4, equation (7.3)). However, by the definition of $V^{N, \tilde{T}}$, its maximum equals to the width of the range of $X^{x, y ; N, \tilde{T}}$, that is, the difference between the maximum and the minimum of $X^{x, y ; N, \tilde{T}}$. Therefore, as in the proof of Proposition 4.2 , the problem at hand reduces to the estimate (4.7).

We continue with some auxiliary convergence results that serve as building blocks in the proof of Theorem 2.1. Consider a sequence of independent random variables $\zeta(m), m \in \mathbb{N}$ which satisfies the following four conditions:

(4.20) $\forall z>0$ :

$$
\lim _{N \rightarrow \infty} N^{-1 / 6} \sum_{m=N-\left\lfloor N^{1 / 3} z\right\rfloor}^{N}|\mathbb{E}[\zeta(m)]|=0,
$$

(4.21) $\exists s_{\zeta} \geq 0$ :

$$
\lim _{N \rightarrow \infty} N^{-1 / 3} \sum_{m=N-\left\lfloor N^{1 / 3} z\right\rfloor}^{N} \mathbb{E}\left[\zeta(m)^{2}\right]=s_{\zeta}^{2} z, \quad z>0,
$$


(4.22) $\forall z>0: \quad \lim _{N \rightarrow \infty} N^{-1 / 2} \sum_{m=N-\left\lfloor N^{1 / 3} z\right\rfloor}^{N} \mathbb{E}\left[|\zeta(m)|^{3}\right]=0$

(4.23) $\exists C>0,0<\gamma<2 / 3: \quad \mathbb{E}\left[|\zeta(m)|^{\ell}\right] \leq C^{\ell} \ell^{\gamma \ell}, \quad m, \ell \in \mathbb{N}$.

Note that (4.20)-(4.23) automatically hold for the choices $\zeta(m)=\mathfrak{a}(m), m \in \mathbb{N}$ and $\zeta(m)=\xi(m), m \in \mathbb{N}$ by Assumption 2.1. In other words, (4.20)-(4.23) are somewhat weaker than conditions (a)-(c) of Assumption 2.1. Before proceeding further let us observe the following simple consequences of (4.23).

LEMMA 4.1. Suppose that for a random variable $\zeta$ there are constants $C>0$ and $0<\gamma<2 / 3$ such that $\mathbb{E}\left[|\zeta|^{\ell}\right] \leq C^{\ell} \ell^{\gamma \ell}$ for all $\ell \in \mathbb{N}$. Then there exist $C^{\prime}>0$ and $2<\gamma^{\prime}<3$ depending only on $C$ and $\gamma$ such that

$$
\begin{aligned}
\mathbb{E}\left[e^{v \zeta}\right] & \leq \exp \left(v \mathbb{E}[\zeta]+C^{\prime}\left(v^{2}+|v|^{\gamma^{\prime}}\right)\right), \quad v \in \mathbb{R} \\
\mathbb{E}\left[|1+v \zeta|^{\ell}\right] & \leq \exp \left(|v| \ell|\mathbb{E}[\zeta]|+C^{\prime}\left(v^{2} \ell^{2}+|v|^{\gamma^{\prime}} \ell^{\gamma^{\prime}}\right)\right), \quad v \in \mathbb{R}, \ell \in \mathbb{N}
\end{aligned}
$$

PROOF. The proof of (4.24) follows closely that of [65], Lemma 5.5, implication 2 . $\Rightarrow 4$. We make the following assumptions without loss of generality: $\gamma>1 / 2 ; \mathbb{E}[\zeta]=0$ (otherwise we can apply (4.24) for $\zeta-\mathbb{E}[\zeta]$ which satisfies the same moment bounds as $\zeta$, just with $C$ replaced by $2 C$ ); and $C=1$ [since we can use (4.24) for $\zeta / C$ when $C>1]$. Under these assumptions, we expand the exponential function on the left-hand side of (4.24) and use the moment bounds for $\zeta$ together with the estimate $\ell ! \geq(\ell / e)^{\ell}$ to get

$$
\mathbb{E}\left[e^{v \zeta}\right] \leq 1+\sum_{\ell=2}^{\infty}\left(\frac{e|v|}{\ell^{1-\gamma}}\right)^{\ell}
$$

On the other hand, an expansion of the exponential function on the right-hand side of (4.24) and the bound $\hat{\ell} ! \leq \hat{\ell}^{\hat{\ell}}$ yield

$$
\exp \left(C^{\prime}\left(v^{2}+|v|^{\gamma^{\prime}}\right)\right) \geq 1+\sum_{\hat{\ell}=1}^{\infty}\left(\frac{C^{\prime}\left(v^{2}+|v|^{\gamma^{\prime}}\right)}{\hat{\ell}}\right)^{\hat{\ell}}
$$

Next, for every $\ell \geq\left\lceil\frac{3}{2-3 \gamma}\right\rceil=: \ell_{*}$, we consider $\hat{\ell}:=\lfloor(1-\gamma) \ell\rfloor$ and note that each value of $\hat{\ell}$ arises for at most three different values of $\ell$ thanks to $1-\gamma>1 / 3$. We pick $\frac{\ell_{*}}{(1-\gamma) \ell_{*}-1}<\gamma^{\prime}<3$ arbitrarily and observe that, in particular, $\gamma^{\prime}>2$ due to $\gamma>1 / 2$. Moreover, with $\alpha_{\ell}:=\frac{\gamma^{\prime} \hat{\ell}-\ell}{\left(\gamma^{\prime}-2\right) \hat{\ell}} \in(0,1)$ (recall the lower bound on $\gamma^{\prime}$, as 
well as $\gamma>1 / 2$ ) and for any $C^{\prime} \geq 1$, we have the estimates

$$
\begin{aligned}
\left(\frac{C^{\prime}\left(v^{2}+|v|^{\gamma^{\prime}}\right)}{\hat{\ell}}\right)^{\hat{\ell}} & \geq \frac{\left(C^{\prime}\right)^{\hat{\ell}}}{(1-\gamma)^{(1-\gamma) \ell} \ell^{(1-\gamma) \ell}}\left(\alpha_{\ell}|v|^{2 \hat{\ell}}+\left(1-\alpha_{\ell}\right)|v|^{\gamma^{\prime} \hat{\ell}}\right) \\
& \geq \frac{\left(C^{\prime}\right)^{(1-\gamma) \ell-1}}{(1-\gamma)^{(1-\gamma) \ell} \ell^{(1-\gamma) \ell}}|v|^{2 \hat{\ell} \alpha_{\ell}+\gamma^{\prime} \hat{\ell}\left(1-\alpha_{\ell}\right)} \\
& =\left(\frac{\left(C^{\prime}\right)^{1-\gamma-1 / \ell}|v|}{(1-\gamma)^{1-\gamma} \ell^{1-\gamma}}\right)^{\ell}
\end{aligned}
$$

In particular, we see that, as soon as $\frac{\left(C^{\prime}\right)^{1-\gamma-1 / \ell_{*}}}{(1-\gamma)^{1-\gamma}}>e$, the $\hat{\ell}$ th term of the sum in (4.27) is greater than the $\ell$ th term of the sum in (4.26) for all $\ell \geq \ell_{*}$. Therefore, by increasing the value of $C^{\prime}$ sufficiently, we can make sure that (4.24) holds.

We now turn to the proof of (4.25). We may again assume without loss of generality that $\mathbb{E}[\zeta]=0$, since otherwise we can use the binomial theorem and the bound (4.25) for $\bar{\zeta}:=\zeta-\mathbb{E}[\zeta]$ to derive the chain of estimates

$$
\begin{aligned}
\mathbb{E}\left[|1+v \zeta|^{\ell}\right] & \leq \sum_{\ell_{1}=0}^{\ell}\left(\begin{array}{l}
\ell \\
\ell_{1}
\end{array}\right) \mathbb{E}\left[|1+v \bar{\zeta}|^{\ell-\ell_{1}}\right](|v||\mathbb{E}[\zeta]|)^{\ell_{1}} \\
& \leq \sum_{\ell_{1}=0}^{\ell}\left(\begin{array}{l}
\ell \\
\ell_{1}
\end{array}\right) e^{C^{\prime}\left(v^{2}\left(\ell-\ell_{1}\right)^{2}+|v|^{\gamma^{\prime}}\left(\ell-\ell_{1}\right)^{\gamma^{\prime}}\right)}(|v||\mathbb{E}[\zeta]|)^{\ell_{1}} \\
& \leq e^{C^{\prime}\left(v^{2} \ell^{2}+|v|^{\gamma^{\prime}} \ell^{\gamma^{\prime}}\right)}(1+|v||\mathbb{E}[\zeta]|)^{\ell},
\end{aligned}
$$

where the last expression is less or equal to the right-hand side of (4.25). When $\mathbb{E}[\zeta]=0$, we may also assume without loss of generality that $\ell$ is even, since for odd $\ell=2 \ell_{1}+1$ we can apply Jensen's inequality and the bound (4.25) with $\ell=2 \ell_{1}+2$ to find

$$
\begin{aligned}
\mathbb{E}\left[|1+v \zeta|^{2 \ell_{1}+1}\right] & \leq \mathbb{E}\left[|1+v \zeta|^{2 \ell_{1}+2}\right]^{\frac{2 \ell_{1}+1}{2 \ell_{1}+2}} \\
& \leq e^{C^{\prime}\left(v^{2}\left(2 \ell_{1}+2\right)\left(2 \ell_{1}+1\right)+|v|^{\gamma^{\prime}}\left(2 \ell_{1}+2\right)^{\gamma^{\prime}-1}\left(2 \ell_{1}+1\right)\right)} \\
& \leq \exp \left(2^{\gamma^{\prime}-1} C^{\prime}\left(v^{2}\left(2 \ell_{1}+1\right)^{2}+|v|^{\gamma^{\prime}}\left(2 \ell_{1}+1\right)^{\gamma^{\prime}}\right)\right) .
\end{aligned}
$$

In particular, it suffices to increase the constant $C^{\prime}$ in (4.25) for even $\ell$ by a factor of $2^{\gamma^{\prime}-1}$ to obtain (4.25) for all $\ell \in \mathbb{N}$.

For even $\ell$, we may drop the absolute value on the left-hand side of (4.25) and use the binomial theorem together with $\mathbb{E}[\zeta]=0,\left(\begin{array}{l}\ell \\ \ell_{1}\end{array}\right) \leq \frac{\ell^{\ell_{1}}}{\ell_{1} !} \leq \ell^{\ell_{1}} /\left(\frac{\ell_{1}}{e}\right)^{\ell_{1}}$, and the moment bounds for $\zeta$ to get

$$
\mathbb{E}\left[|1+v \zeta|^{\ell}\right] \leq 1+\sum_{\ell_{1}=2}^{\ell} \frac{\ell^{\ell_{1}}}{\left(\frac{\ell_{1}}{e}\right)^{\ell_{1}}}|v|^{\ell_{1}} C^{\ell_{1}} \ell_{1}^{\gamma \ell_{1}} \leq 1+\sum_{\ell_{1}=2}^{\infty}\left(\frac{e \ell|v| C}{\ell_{1}^{1-\gamma}}\right)^{\ell_{1}} .
$$


The latter expression equals to the right-hand side of (4.26) with $|v|$ replaced by $\ell|v| C$, so that we can bound it from above by the right-hand side of (4.24) with $v$ replaced by $\ell v C$. As a result, we obtain the estimate (4.25) with the same $\gamma^{\prime}$ as in (4.24) once we suitably enlarge $C^{\prime}$.

We proceed to the first auxiliary convergence result.

Proposition 4.4. Let $x, y \in \mathbb{R}$ and $T_{N}>0, N \in \mathbb{N}$ be such that $\sup _{N} \mid T_{N}-$ $T \mid N^{2 / 3}<\infty$ for some $T>0, T_{N} N^{2 / 3} \in \mathbb{N}$ for all $N$, and $T_{N} N^{2 / 3}$ has the same parity as $\left\lfloor N-N^{1 / 3} x\right\rfloor-\left\lfloor N-N^{1 / 3} y\right\rfloor$. Then, under (4.20)-(4.22), the pair

$$
\left(N^{-1 / 3}\left(N-X^{x, y ; N, T_{N}}\right), \sum_{h \in N^{-1 / 3}\left(\mathbb{Z}_{\geq 0}+1 / 2\right)} L_{h}\left(X^{x, y ; N, T_{N}}\right) \frac{\zeta\left(\left\lfloor N-N^{1 / 3} h\right\rfloor\right)}{N^{1 / 6}}\right)
$$

converges in the limit $N \rightarrow \infty$ in distribution to

$$
\left(B^{x, y}, s_{\zeta} \int_{0}^{\infty} L_{a}\left(B^{x, y}\right) \mathrm{d} W_{\zeta}(a)\right)
$$

Here, $W_{\zeta}$ is a standard Brownian motion, and the convergence of the first argument is with respect to the topology of uniform convergence on $[0, T]$ (if $T_{N}<T$, we extend $X^{x, y ; N, T_{N}}$ to $[0, T]$ by extending its last linear segment).

PROOF. We work throughout under the coupling of Proposition 4.1 [note that we can enlarge the probability space there further to support copies of $\zeta(m), m \in$ $\mathbb{N}$ and $W_{\zeta}$. For every fixed $N \in \mathbb{N}$, we consider the conditional characteristic function

$$
\mathbb{E}_{\zeta}\left[\exp \left(\mathbf{i} u \sum_{h \in N^{-1 / 3}\left(\mathbb{Z}_{\geq 0}+1 / 2\right)} L_{h}\left(X^{x, y ; N, T_{N}}\right) \frac{\zeta\left\lfloor N-N^{1 / 3} h\right\rfloor}{N^{1 / 6}}\right)\right], \quad u \in \mathbb{R},
$$

where the expectation is taken with respect to the $\zeta(m), m \in \mathbb{N}$ (so that the randomness in the result is coming only from $\left.X^{x, y ; N, T_{N}}\right)$. At this point, it suffices to show that, for each $u \in \mathbb{R}$, the difference between (4.30) and the conditional characteristic function

$$
\mathbb{E}_{W_{\zeta}}\left[\exp \left(\mathbf{i} u s_{\zeta} \int_{0}^{\infty} L_{a}\left(B^{x, y}\right) \mathrm{d} W_{\zeta}(a)\right)\right], \quad u \in \mathbb{R}
$$

tends to 0 almost surely [in (4.31), the expectation is taken with respect to $W_{\zeta}$, and the randomness in the result is coming only from $B^{x, y}$. Indeed, the convergence in distribution of (4.28) is equivalent to the pointwise convergence of the 
characteristic functions

$$
\begin{aligned}
& \mathbb{E}\left[\operatorname { e x p } \left(\mathbf{i} r F\left(N^{-1 / 3}\left(N-X^{x, y ; N, T_{N}}\right)\right)\right.\right. \\
& \left.\left.\quad+\mathbf{i} u \sum_{h \in N^{-1 / 3}\left(\mathbb{Z}_{\geq 0}+1 / 2\right)} L_{h}\left(X^{x, y ; N, T_{N}}\right) \frac{\zeta\left(\left\lfloor N-N^{1 / 3} h\right\rfloor\right)}{N^{1 / 6}}\right)\right] \\
& \longrightarrow \mathbb{E}\left[\exp \left(\mathbf{i} r F\left(B^{x, y}\right)+\mathbf{i} u s_{\zeta} \int_{0}^{\infty} L_{a}\left(B^{x, y}\right) \mathrm{d} W_{\zeta}(a)\right)\right], \quad(r, u) \in \mathbb{R}^{2}
\end{aligned}
$$

for all bounded continuous functionals $F$ on the space of continuous functions on $[0, T]$ endowed with the topology of uniform convergence. The latter convergence follows directly from the dominated convergence theorem, (4.3), and the almost sure convergence to 0 of the difference between (4.30) and (4.31).

The rest of the proof is devoted to showing that the difference between (4.30) and (4.31) tends to 0 almost surely in the limit $N \rightarrow \infty$. Given $B^{x, y}$, the random variable $s_{\zeta} \int_{0}^{\infty} L_{a}\left(B^{x, y}\right) \mathrm{d} W(a)$ is simply a normal random variable with mean zero and variance $s_{\zeta}^{2} \int_{0}^{\infty} L_{a}\left(B^{x, y}\right)^{2} \mathrm{~d} a$; and given $X^{x, y ; N, T_{N}}$, the summands in $\sum_{h \in N^{-1 / 3}\left(\mathbb{Z}_{\geq 0}+1 / 2\right)} L_{h}\left(X^{\left.x, y ; N, T_{N}\right)} \frac{\zeta\left(\left\lfloor N-N^{1 / 3} h\right\rfloor\right)}{N^{1 / 6}}\right.$ are independent. Therefore, the desired convergence will be a consequence of the central limit theorem in the form of the upper bound of [6], Theorem 8.4, provided we can prove the following three almost sure convergences as $N \rightarrow \infty$ :

$$
\begin{gathered}
\sum_{h \in N^{-1 / 3}\left(\mathbb{Z}_{\geq 0}+1 / 2\right)} \mathbb{E}_{\zeta}\left[L_{h}\left(X^{x, y ; N, T_{N}}\right) \frac{\zeta\left(\left\lfloor N-N^{1 / 3} h\right\rfloor\right)}{\left.N^{1 / 6}\right]} \rightarrow 0,\right. \\
\sum_{h \in N^{-1 / 3}\left(\mathbb{Z}_{\geq 0}+1 / 2\right)} \mathbb{E}_{\zeta}\left[\left(L_{h}\left(X^{x, y ; N, T_{N}}\right) \frac{\zeta\left(\left\lfloor N-N^{1 / 3} h\right\rfloor\right)}{N^{1 / 6}}\right)^{2}\right] \\
\longrightarrow s_{\zeta}^{2} \int_{0}^{\infty}\left[L_{a}\left(B^{x, y}\right)\right]^{2} \mathrm{~d} a, \\
\sum_{h \in N^{-1 / 3}\left(\mathbb{Z}_{\geq 0}+1 / 2\right)} \mathbb{E}_{\zeta}\left[\left\lfloor\left. L_{h}\left(X^{x, y ; N, T_{N}}\right) \frac{\zeta\left(\left\lfloor N-N^{1 / 3} h\right\rfloor\right)}{N^{1 / 6}}\right|^{3}\right] \longrightarrow 0 .\right.
\end{gathered}
$$

As before, $\mathbb{E}_{\zeta}$ denotes the expectation with respect to the $\zeta(m), m \in \mathbb{N}$. The convergences (4.33) and (4.34) pin down the limiting mean and variance, whereas the convergence (4.35) is the well-known Lyapunov condition for the central limit theorem.

The coupling of Proposition 4.1 reveals that, with probability one, the $L_{h}\left(X^{x, y ; N, T_{N}}\right)$ are uniformly bounded in $h \in \mathbb{R}, N \in \mathbb{N}$ and vanish for all $h$ large enough, so that (4.33) follows from (4.20). Similarly, (4.35) follows from (4.22). To obtain (4.34), we combine the simple inequality $\left|r_{1}^{2}-r_{2}^{2}\right| \leq 2 \max \left(r_{1}, r_{2}\right) \mid r_{1}-$ 
$r_{2} \mid, r_{1}, r_{2}>0$ with Proposition 4.1 to conclude that

$$
\begin{aligned}
& \mid \sum_{h \in N^{-1 / 3}\left(\mathbb{Z}_{\geq 0}+1 / 2\right)} \mathbb{E}_{\zeta}\left[\left(L_{h}\left(X^{x, y ; N, T_{N}}\right) \frac{\zeta\left(\left\lfloor N-N^{1 / 3} h\right\rfloor\right)}{N^{1 / 6}}\right)^{2}\right] \\
& -\sum_{h \in N^{-1 / 3}\left(\mathbb{Z}_{\geq 0}+1 / 2\right)} \frac{L_{h}\left(B^{x, y}\right)^{2} \mathbb{E}\left[\zeta\left(\left\lfloor N-N^{1 / 3} h\right\rfloor\right)^{2}\right]}{N^{1 / 3}} \mid \\
& \leq 2 \max _{h \in N^{-1 / 3}\left(\mathbb{Z}_{\geq 0+1 / 2}\right)} \max \left(L_{h}\left(X^{x, y ; N, T_{N}}\right), L_{h}\left(B^{x, y}\right)\right) \mathcal{C} N^{-1 / 16} \\
& \cdot \frac{1}{N^{1 / 3}} \sum_{N^{-1 / 3}\left(\mathbb{Z}_{\geq 0}+1 / 2\right) \ni h \leq \max \left(\| X^{\left.x, y ; N, T_{N}\left\|_{\infty},\right\| B^{x, y} \|_{\infty}\right)}\right.} \mathbb{E}\left[\zeta\left(\left\lfloor N-N^{1 / 3} h\right\rfloor\right)^{2}\right] .
\end{aligned}
$$

The latter expression tends to 0 almost surely in the limit $N \rightarrow \infty$, since $h \mapsto$ $L_{h}\left(X^{x, y ; N, T_{N}}\right)$ and $X^{x, y ; N, T_{N}}$ are uniformly bounded in $N \in \mathbb{N}$ (Proposition 4.1) and (4.21) holds. It remains to observe the almost sure convergence

$$
\sum_{h \in N^{-1 / 3}\left(\mathbb{Z}_{\geq 0}+1 / 2\right)} \frac{\left[L_{h}\left(B^{x, y}\right)\right]^{2} \mathbb{E}\left[\zeta\left(\left\lfloor N-N^{1 / 3} h\right\rfloor\right)^{2}\right]}{N^{1 / 3}} \longrightarrow s_{\zeta}^{2} \int_{0}^{\infty}\left[L_{a}\left(B^{x, y}\right)\right]^{2} \mathrm{~d} a
$$

as $N \rightarrow \infty$, which follows from (4.21) and the uniform continuity of $a \mapsto$ $L_{a}\left(B^{x, y}\right)^{2}$.

Next, we present an extension of Proposition 4.4 which allows the endpoints $x, y$ to be random. Given probability measures $\lambda, \mu$ on $[0, \infty)$ and $\tilde{T} \in N^{-2 / 3} \mathbb{N}$, we write $X^{\lambda, \mu ; N, \tilde{T}}$ for the random walk bridge whose endpoints $x, y$ are chosen independently according to the images of $\lambda, \mu$ under the map $z \mapsto\left\lfloor N-N^{1 / 3} z\right\rfloor$ and which makes $\tilde{T} N^{2 / 3}$ steps of size \pm 1 if $\tilde{T} N^{2 / 3}$ has the same parity as $\lfloor N-$ $\left.N^{1 / 3} y\right\rfloor-\left\lfloor N-N^{1 / 3} x\right\rfloor$ and $\tilde{T} N^{2 / 3}-1$ steps of size \pm 1 otherwise. For $x, y$ for which there are no bridges of the type described (because the endpoints are too far apart), we instead let $X^{\lambda, \mu ; N, \tilde{T}}$ be a straight line connecting $x$ to $y$ in time $\tilde{T} N^{2 / 3}$. Similarly, we write $B^{\lambda, \mu}$ for a Brownian bridge whose endpoints $x, y$ are selected independently according to $\lambda, \mu$, respectively. We further extend the setup of Proposition 4.4 by allowing for multiple bridges, considering local times at both (scaled) integer and half-integer points, and adding a second sequence $\hat{\zeta}(m)$, $m \in \mathbb{N}$.

Proposition 4.5. Fix an $R \in \mathbb{N}$, probability measures $\lambda_{1}, \lambda_{2}, \ldots, \lambda_{R}, \mu_{1}$, $\mu_{2}, \ldots, \mu_{R}$ on $[0, \infty)$, and $T_{N}, N \in \mathbb{N}$ such that $\sup _{N}\left|T_{N}-T\right| N^{2 / 3}<\infty$ for some $T>0$ and $T_{N} N^{2 / 3} \in \mathbb{N}, N \in \mathbb{N}$. For each $N \in \mathbb{N}$, let $X^{\lambda_{1}, \mu_{1} ; N, T_{N}}, \ldots$, $X^{\lambda_{R}, \mu_{R} ; N, T_{N}}$ be independent random walk bridges as described above. Then, for 
any two independent sequences $\zeta(m), m \in \mathbb{N}$ and $\hat{\zeta}(m), m \in \mathbb{N}$ satisfying the assumptions (4.20), (4.21) and (4.22), the random vector

$$
\begin{gathered}
\left(N^{-1 / 3}\left(N-X^{\lambda_{r}, \mu_{r} ; N, T_{N}}\right),\right. \\
\sum_{N^{-1 / 3}(\mathbb{Z}+1 / 2) \ni h \leq N^{-1 / 3}(N-1)} L_{h}\left(X^{\lambda_{r}, \mu_{r} ; N, T_{N}}\right) \frac{\zeta\left(\left\lfloor N-N^{1 / 3} h\right\rfloor\right)}{N^{1 / 6}}, \\
\left.\sum_{N^{-1 / 3} \mathbb{Z} \ni h \leq N^{-1 / 3}(N-1)} L_{h}\left(X^{\lambda_{r}, \mu_{r} ; N, T_{N}}\right) \frac{\hat{\zeta}\left(\left\lfloor N-N^{1 / 3} h\right\rfloor\right)}{N^{1 / 6}}\right)_{r=1}^{R}
\end{gathered}
$$

converges in the limit $N \rightarrow \infty$ in distribution to

$$
\left(B^{\lambda_{r}, \mu_{r}}, s_{\zeta} \int_{0}^{\infty} L_{a}\left(B^{\lambda_{r}, \mu_{r}}\right) \mathrm{d} W_{\zeta}(a), s_{\hat{\zeta}} \int_{0}^{\infty} L_{a}\left(B^{\lambda_{r}, \mu_{r}}\right) \mathrm{d} W_{\hat{\zeta}}(a)\right)_{r=1}^{R},
$$

where $B^{\lambda_{1}, \mu_{1}}, B^{\lambda_{2}, \mu_{2}}, \ldots, B^{\lambda_{R}, \mu_{R}}$ are independent Brownian bridges on $[0, T]$ as described above, and $W_{\zeta}, W_{\hat{\zeta}}$ are independent standard Brownian motions. In addition, the following convergences in distribution with respect to the Skorokhod topology occur jointly with the convergence of (4.36) to (4.37):

$$
\begin{array}{ll}
\frac{1}{s_{\zeta} N^{1 / 6}} \sum_{m=N-\left\lfloor a N^{1 / 3}\right\rfloor}^{N} \zeta(m) \longrightarrow W_{\zeta}(a), \quad N \rightarrow \infty, \\
\frac{1}{s_{\hat{\zeta}} N^{1 / 6}} \sum_{m=N-\left\lfloor a N^{1 / 3}\right\rfloor}^{N} \hat{\zeta}(m) \longrightarrow W_{\hat{\zeta}}(a), \quad N \rightarrow \infty .
\end{array}
$$

PROOF. For $R=1$, the replacement of deterministic endpoints of Proposition 4.4 by random ones can be dealt with by simply integrating with respect to $\lambda_{1}$ and $\mu_{1}$. Note that, since convergence in distribution amounts to convergence of expectations of bounded continuous functionals, we can use the dominated convergence theorem to justify the convergence of the integrals of such. The sum over (scaled) integers in (4.36) can be analyzed in the same way as the sum over (scaled) half-integers, as Proposition 4.1 does not distinguish between integers and half-integers. For $R>1$, one can repeat the proof of Proposition 4.4, replacing the central limit theorem invoked there by its multidimensional version. The same central limit theorem yields the additional convergences (4.38), (4.39) in the sense of convergence of finite-dimensional distributions and the tightness result needed to upgrade the latter to convergence of processes is standard (see, e.g., [8], Problem 8.4 and proof of Theorem 8.1). 
4.2. Leading order terms in Theorem 2.1. We fix a $T>0$, an interval $\mathcal{A} \subset$ $\mathbb{R}_{\geq 0}$, and functions $f, g \in \mathcal{S}$. Moreover, for $N \in \mathbb{N}$ and $\tilde{T}>0$ such that $\tilde{T} N^{2 / 3} \in \mathbb{N}$ has the same parity as $\left\lfloor N-N^{1 / 3} x\right\rfloor-\left\lfloor N-N^{1 / 3} y\right\rfloor$, we let

$$
\Xi(x, y ; N, \tilde{T}):=\frac{N^{1 / 3}}{2^{\tilde{T} N^{2 / 3}}}\left(\begin{array}{c}
\tilde{T} N^{2 / 3} \\
\frac{1}{2}\left(\tilde{T} N^{2 / 3}+\left\lfloor N-N^{1 / 3} y\right\rfloor-\left\lfloor N-N^{1 / 3} x\right\rfloor\right)
\end{array}\right) .
$$

Note that $\Xi(x, y ; N, \tilde{T})$ gives the (normalized) number of possible trajectories of $X^{x, y ; N, \tilde{T}}$. For each $N \in \mathbb{N}$, we further define the random variables $\operatorname{Sc}(N)$ (for "scalar product") and $\operatorname{Tr}(N)$ (for "trace") by

$$
\begin{aligned}
\operatorname{Sc}(N)= & \frac{1}{2} \int_{0}^{N^{2 / 3}} \int_{0}^{N^{2 / 3} f(x) g(y)} \\
& \cdot \sum_{j=0}^{\left\lfloor T N^{2 / 3}\right\rfloor}\left(\Xi ( x , y ; N , ( \lfloor T N ^ { 2 / 3 } \rfloor - j - \varepsilon _ { j , x , y } ) N ^ { - 2 / 3 } ) \mathbb { E } _ { X } \left[\mathbf{1}_{\{\forall t: X(t) \leq N\}}\right.\right. \\
& \cdot \prod^{\left\lfloor N^{2 / 3}\right\rfloor-j-\varepsilon_{j, x, y}} \frac{\sqrt{X\left(i N^{-2 / 3}\right) \wedge X\left((i-1) N^{-2 / 3}\right)}}{\sqrt{N}} \\
& \cdot\left(1+\frac{\xi\left(X\left(i N^{-2 / 3}\right) \wedge X\left((i-1) N^{-2 / 3}\right)\right)}{\left.\sqrt{X\left(i N^{-2 / 3}\right) \wedge X\left((i-1) N^{-2 / 3}\right)}\right)}\right. \\
& \left.\left.\cdot \frac{1}{(2 \sqrt{N})^{j}} \sum_{0 \leq i_{1} \leq \cdots \leq i_{j} \leq\left\lfloor T N^{2 / 3}\right\rfloor-j-\varepsilon_{j, x, y}} \prod_{j^{\prime}=1}^{j} \mathfrak{a}\left(X\left(i_{j^{\prime}} N^{-2 / 3}\right)\right)\right]\right) \mathrm{d} x \mathrm{~d} y,
\end{aligned}
$$$$
\operatorname{Tr}(N)=\frac{1}{2} \int_{0}^{N^{2 / 3}} \sum_{j=0}^{\left\lfloor T N^{2 / 3}\right\rfloor}\left(\Xi\left(x, x ; N,\left(\left\lfloor T N^{2 / 3}\right\rfloor-j-\varepsilon_{j, x, x}\right) N^{-2 / 3}\right)\right.
$$$$
\cdot \mathbb{E}_{X}\left[\mathbf{1}_{\{\forall t: X(t) \leq N\}} \prod_{i=1}^{\left\lfloor T N^{2 / 3}\right\rfloor-j-\varepsilon_{j, x, x}} \frac{\sqrt{X\left(i N^{-2 / 3}\right) \wedge X\left((i-1) N^{-2 / 3}\right)}}{\sqrt{N}}\right.
$$

$$
\begin{aligned}
& \cdot\left(1+\frac{\xi\left(X\left(i N^{-2 / 3}\right) \wedge X\left((i-1) N^{-2 / 3}\right)\right)}{\sqrt{X\left(i N^{-2 / 3}\right) \wedge X\left((i-1) N^{-2 / 3}\right)}}\right) \\
& \left.\left.\cdot \frac{1}{(2 \sqrt{N})^{j}} \sum_{0 \leq i_{1} \leq \cdots \leq i_{j} \leq\left\lfloor T N^{2 / 3}\right\rfloor-j-\varepsilon_{j, x, x}} \prod_{j^{\prime}=1}^{j} \mathfrak{a}\left(X\left(i_{j^{\prime}} N^{-2 / 3}\right)\right)\right]\right) \mathrm{d} x,
\end{aligned}
$$

where $\epsilon_{j, x, y} \in\{0,1\}$ is such that $\left\lfloor T N^{2 / 3}\right\rfloor-j-\epsilon_{j, x, y}$ is even, and $X$ stands for $X^{x, y ; N,\left(\left\lfloor T N^{2 / 3}\right\rfloor-j-\varepsilon_{j, x, y}\right) N^{-2 / 3}}$ in (4.40) and for $X^{x, x ; N,\left(\left\lfloor T N^{2 / 3}\right\rfloor-j-\varepsilon_{j, x, x}\right) N^{-2 / 3}}$ in (4.41). The discussion of Section 3 shows $\operatorname{Sc}(N)=\left(\pi_{N} f\right)^{\prime} \mathcal{M}(T, N)\left(\pi_{N} g\right)$ and 
$\operatorname{Tr}(N)=\operatorname{Trace}(\mathcal{M}(T, N))$ in the notation of Theorem 2.1. Consequently, Theorem 2.1 can be rephrased as follows.

THEOREM 4.2. Under Assumption 2.1 and as $N \rightarrow \infty$, one has the following convergences for all fixed $T>0, \mathcal{A} \subset \mathbb{R}_{\geq 0}$, and $f, g \in \mathcal{S}$ :

$$
\operatorname{Sc}(N) \rightarrow \frac{1}{\sqrt{2 \pi T}} \int_{0}^{\infty} \int_{0}^{\infty} f(x) g(y) \exp \left(-\frac{(x-y)^{2}}{2 T}\right) \mathbb{E}_{B^{x, y}}\left[\mathbf{1}_{\left\{\forall t: B^{x, y}(t) \geq 0\right\}}\right.
$$

$$
\left.\cdot \exp \left(-\frac{1}{2} \int_{0}^{T} B^{x, y}(t) \mathrm{d} t+\frac{1}{\sqrt{\beta}} \int_{0}^{\infty} L_{a}\left(B^{x, y}\right) \mathrm{d} W(a)\right)\right] \mathrm{d} x \mathrm{~d} y,
$$

$$
\begin{aligned}
\operatorname{Tr}(N) \rightarrow & \frac{1}{\sqrt{2 \pi T}} \int_{0}^{\infty} \mathbb{E}_{B^{x, x}}\left[\mathbf{1}_{\left\{\forall t: B^{x, x}(t) \geq 0\right\}}\right. \\
& \left.\cdot \exp \left(-\frac{1}{2} \int_{0}^{T} B^{x, x}(t) \mathrm{d} t+\frac{1}{\sqrt{\beta}} \int_{0}^{\infty} L_{a}\left(B^{x, x}\right) \mathrm{d} W(a)\right)\right] \mathrm{d} x,
\end{aligned}
$$

and

$$
\sqrt{\beta} N^{-1 / 6} \sum_{m=N-\left\lfloor N^{1 / 3} a\right\rfloor}^{N}\left(\xi(m)+\frac{\mathfrak{a}(m)}{2}\right) \rightarrow W(a) .
$$

The convergences (4.42) and (4.43) hold in distribution and in the sense of moments, and the same apply jointly to any finite collection of $T$ ' $s, f$ 's and $g$ 's. The convergence in (4.44) is in distribution with respect to the Skorokhod topology and takes place jointly with both distributional convergences (4.42), (4.43) (also for finitely many $T$ 's, $f$ 's and $g$ 's).

The proof of Theorem 4.2 is divided into two parts. First, we identify the leading order terms in $\operatorname{Sc}(N)$ and $\operatorname{Tr}(N)$ and compute their limits in Proposition 4.6. Then we analyze the corresponding remainder terms and prove that they vanish asymptotically in Lemmas 4.6, 4.7 and 4.8. In Section 4.3, we explain how these results lead to Theorem 4.2 .

We start by defining $\mathrm{Sc}^{(j)}(N ; K, \underline{R}, \bar{R})$ and $\operatorname{Tr}^{(j)}(N ; K, \underline{R}, \bar{R})$ which will turn out to be the leading order contributions to the $j$ th terms in $\operatorname{Sc}(N)$ and $\operatorname{Tr}(N)$, respectively. For any $K \in[0, \infty), \underline{R} \in[-\infty, 0], \bar{R} \in[0, \infty]$ (we allow infinite values for $\underline{R}, \bar{R})$, set

$$
\begin{aligned}
& \mathrm{Sc}^{(j)}(N ; K, \underline{R}, \bar{R}) \\
& :=\frac{1}{2} \int_{0}^{K} \int_{0}^{K} f(x) g(y) \Xi\left(x, y ; N, T_{N}\right)
\end{aligned}
$$

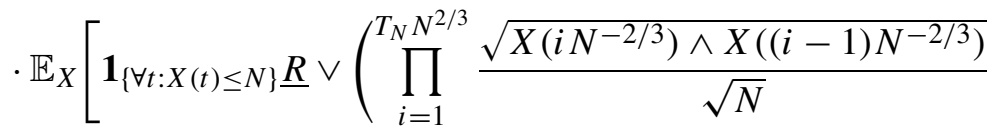




$$
\begin{aligned}
& \cdot\left(1+\frac{\xi\left(X\left(i N^{-2 / 3}\right) \wedge X\left((i-1) N^{-2 / 3}\right)\right)}{\sqrt{X\left(i N^{-2 / 3}\right) \wedge X\left((i-1) N^{-2 / 3}\right)}}\right) \\
& \left.\left.\cdot \frac{\left(\sum_{i^{\prime}=0}^{T_{N} N^{2 / 3}} \mathfrak{a}\left(X\left(i^{\prime} N^{-2 / 3}\right)\right)\right)^{j}}{j !(2 \sqrt{N})^{j}}\right) \wedge \bar{R}\right] \mathrm{d} x \mathrm{~d} y,
\end{aligned}
$$

$\operatorname{Tr}^{(j)}(N ; K, \underline{R}, \bar{R})$

$$
:=\frac{1}{2} \int_{0}^{K} \Xi\left(x, x ; N, T_{N}\right)
$$

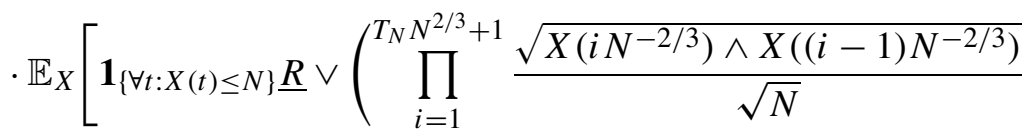

$$
\begin{aligned}
& \cdot\left(1+\frac{\xi\left(X\left(i N^{-2 / 3}\right) \wedge X\left((i-1) N^{-2 / 3}\right)\right)}{\sqrt{X\left(i N^{-2 / 3}\right) \wedge X\left((i-1) N^{-2 / 3}\right)}}\right) \\
& \left.\left.\cdot \frac{\left(\sum_{i^{\prime}=0}^{T_{N} N^{2 / 3}} \mathfrak{a}\left(X\left(i^{\prime} N^{-2 / 3}\right)\right)\right)^{j}}{j !(2 \sqrt{N})^{j}}\right) \wedge \bar{R}\right] \mathrm{d} x
\end{aligned}
$$

where $X$ is a shorthand for $X^{x, y ; N,\left(\left\lfloor T N^{2 / 3}\right\rfloor-j-\varepsilon_{j, x, y}\right) N^{-2 / 3}}$ in (4.45) and for $X^{x, x ; N,\left(\left\lfloor T N^{2 / 3}\right\rfloor-j-\varepsilon_{j, x, x}\right) N^{-2 / 3}}$ in (4.46); $\underline{R} \vee \cdot \wedge \bar{R}$ is an abbreviation for $\max (\underline{R}$, $\min (\cdot, \bar{R}))$; and $T_{N}$ is given by $\left(\left\lfloor T N^{2 / 3}\right\rfloor-j-\varepsilon_{j, x, y}\right) N^{-2 / 3}$ in (4.45) and by $\left(\left\lfloor T N^{2 / 3}\right\rfloor-j-\varepsilon_{j, x, x}\right) N^{-2 / 3}$ in (4.46). The formulas for $T_{N}$ are not important for the arguments below: we only use that $\sup _{N}\left|T_{N}-T\right| N^{2 / 3}<\infty$ for every fixed $j$ in Proposition 4.6 and that $\sup _{N} T_{N}<\infty$ in Lemmas 4.6, 4.7 and 4.8.

PROPOSITION 4.6. In the situation of Theorem 4.2, the following convergences hold as $N \rightarrow \infty$ for any finite collection of $j$ 's and any fixed $K \in[0, \infty)$, $\underline{R} \in[-\infty, 0], \bar{R} \in[0, \infty]$ (the modes of convergence are the same as in Theorem 4.2):

$$
\begin{aligned}
\operatorname{Sc}^{(j)}(N ; K, \underline{R}, \bar{R}) \rightarrow & \frac{1}{\sqrt{2 \pi T}} \int_{0}^{K} \int_{0}^{K} f(x) g(y) \exp \left(-\frac{(x-y)^{2}}{2 T}\right) \\
& \cdot \mathbb{E}_{B^{x, y}}\left[\mathbf{1}_{\left\{\forall t: B^{x, y}(t) \geq 0\right\} \underline{R} \vee\left(\operatorname { e x p } \left(-\frac{1}{2} \int_{0}^{T} B^{x, y}(t) \mathrm{d} t\right.\right.}\right. \\
& \left.+s_{\xi} \int_{0}^{\infty} L_{a}\left(B^{x, y}\right) \mathrm{d} W_{\xi}(a)\right) \\
& \left.\left.\cdot \frac{\left(s_{\mathfrak{a}} \int_{0}^{\infty} L_{a}\left(B^{x, y}\right) \mathrm{d} W_{\mathfrak{a}}(a)\right)^{j}}{2^{j} j !}\right) \wedge \bar{R}\right] \mathrm{d} x \mathrm{~d} y, \\
\operatorname{Tr}^{(j)}(N ; K, \underline{R}, \bar{R}) \rightarrow & \frac{1}{\sqrt{2 \pi T}} \int_{0}^{K} \mathbb{E}_{B^{x, x}}\left[\mathbf{1}_{\left\{\forall t: B^{x, x}(t) \geq 0\right\}}\right.
\end{aligned}
$$




$$
\begin{gathered}
\cdot \underline{R} \vee\left(\operatorname { e x p } \left(-\frac{1}{2} \int_{0}^{T} B^{x, x}(t) \mathrm{d} t\right.\right. \\
\left.+s \xi \int_{0}^{\infty} L_{a}\left(B^{x, x}\right) \mathrm{d} W_{\xi}(a)\right) \\
\left.\left.\cdot \frac{\left(s_{\mathfrak{a}} \int_{0}^{\infty} L_{a}\left(B^{x, x}\right) \mathrm{d} W_{\mathfrak{a}}(a)\right)^{j}}{2^{j} j !}\right) \wedge \bar{R}\right] \mathrm{d} x, \\
s_{\xi} N^{-1 / 6} \sum_{h=N-\left\lfloor a N^{1 / 3}\right\rfloor}^{N} \xi(h) \rightarrow W_{\xi}(a), \\
\sum_{\mathfrak{a}} N^{-1 / 6} \sum_{h=N-\left\lfloor a N^{1 / 3}\right\rfloor}^{N} \mathfrak{a}(h) \rightarrow W_{\mathfrak{a}}(a) .
\end{gathered}
$$

We prove Proposition 4.6 in a series of lemmas. In all of their proofs, we only consider $\mathrm{Sc}^{(j)}(N ; K, \underline{R}, \bar{R})$, since the arguments for $\operatorname{Tr}^{(j)}(N ; K, \underline{R}, \bar{R})$ are exactly the same.

LEMMA 4.2. Let $T_{N}, N \in \mathbb{N}$ be such that $\sup _{N}\left|T_{N}-T\right| N^{2 / 3}<\infty$ and $T_{N} N^{2 / 3} \in \mathbb{N}, N \in \mathbb{N}$. Then, uniformly in $x, y$ in any compact set and such that $\left\lfloor N-N^{1 / 3} x\right\rfloor-\left\lfloor N-N^{1 / 3} y\right\rfloor$ has the same parity as $T_{N} N^{2 / 3}$, it holds

$$
\Xi\left(x, y ; N, T_{N}\right) \rightarrow \sqrt{\frac{2}{\pi T}} e^{-(x-y)^{2} /(2 T)}, \quad N \rightarrow \infty .
$$

In addition, there exists a $C>0$ such that

$$
\Xi(x, y ; N, \tilde{T}) \leq C e^{-(x-y)^{2} /(C \tilde{T})}
$$

for all $x, y \in \mathbb{R}, N \in \mathbb{N}$, and $\tilde{T}>0$ with $\tilde{T} N^{2 / 3} \in \mathbb{N}$ being of the same parity as $\left\lfloor N-N^{1 / 3} x\right\rfloor-\left\lfloor N-N^{1 / 3} y\right\rfloor$.

PROOF. The first statement is a special case of the de Moivre-Laplace theorem in the form of [31], Section VII.3, Theorem 1. To obtain the second statement, we distinguish between the cases $(x-y)^{2} / \tilde{T} \leq \frac{4}{3} \log N$ and $(x-y)^{2} / \tilde{T}>\frac{4}{3} \log N$. In the first case, the bound (4.51) follows directly from [31], Section VII.3, Theorem 1, upper bound in (3.14). In the second case and for $x, y, N, \tilde{T}$ such that $\Xi(x, y ; N, \tilde{T})>0$, we apply the nonasymptotic upper bound from the proof of Cramér's theorem (see, e.g., [23], Remark (c) after Theorem 2.2.3) to get

$$
\Xi(x, y ; N, \tilde{T})
$$

$$
\leq 2 N^{1 / 3} \exp \left(-\tilde{T} N^{2 / 3} \Lambda^{*}\left(\frac{1}{2}+\frac{\left|\left\lfloor N-N^{1 / 3} x\right\rfloor-\left\lfloor N-N^{1 / 3} y\right\rfloor\right|}{2 \tilde{T} N^{2 / 3}}\right)\right)
$$


with $\Lambda^{*}(z):=\log 2+z \log z+(1-z) \log (1-z)$. It is easy to check $\Lambda^{*}(1 / 2)=$ $\left(\Lambda^{*}\right)^{\prime}(1 / 2)=0$ and $\left(\Lambda^{*}\right)^{\prime \prime}(z) \geq 4$, which together imply $\Lambda^{*}(z) \geq 2(z-1 / 2)^{2}$. Inserting the latter bound into (4.52) gives (4.51).

Lemma 4.3. Proposition 4.6 holds for $K, \bar{R} \in[0, \infty), \underline{R} \in(-\infty, 0]$.

PROOF. Without loss of generality, we may assume that

$$
f \geq 0, g \geq 0, \quad \int_{0}^{K} f(x) \mathrm{d} x=\int_{0}^{K} g(y) \mathrm{d} y=1,
$$

since otherwise we can decompose $f, g$ into their positive and negative parts and normalize the latter appropriately. Further, we let $\lambda, \mu$ be the probability distributions on $[0, \infty)$ with densities $f \mathbf{1}_{[0, K]}, g \mathbf{1}_{[0, K]}$, respectively. In addition, for every $N \in \mathbb{N}$ and $R \in \mathbb{N}$, we introduce the independent copies $X_{r}^{N}, r=1,2, \ldots, R$ of the random walk bridge $X^{\lambda, \mu ; N, T_{N}}$ (with $T_{N}$ defined in the paragraph preceding Proposition 4.6). Then, thanks to Fubini's Theorem, the $R$ th moment of the random variable $\operatorname{Sc}^{(j)}(N, K, \underline{R}, \bar{R})$ can be expressed as

$$
\begin{aligned}
\mathbb{E}\left[\prod_{r=1}^{R} \Xi\left(X_{r}^{N}(0), X_{r}^{N}\left(T_{N}\right) ; N, T_{N}\right) \mathbf{1}_{\left\{\forall t: X_{r}^{N}(t) \leq N\right\}}\right. \\
\quad \cdot \underline{R} \vee\left(\prod_{i=1}^{T_{N} N^{2 / 3}} \frac{\sqrt{X_{r}^{N}\left(i N^{-2 / 3}\right) \wedge X_{r}^{N}\left((i-1) N^{-2 / 3}\right)}}{\sqrt{N}}\right. \\
\quad \cdot\left(1+\frac{\xi\left(X_{r}^{N}\left(i N^{-2 / 3}\right) \wedge X_{r}^{N}\left((i-1) N^{-2 / 3}\right)\right)}{\sqrt{X_{r}^{N}\left(i N^{-2 / 3}\right) \wedge X_{r}^{N}\left((i-1) N^{-2 / 3}\right)}}\right) \\
\left.\left.\quad \frac{\left(\sum_{i^{\prime}=0}^{T_{N} N^{2 / 3}} \mathfrak{a}\left(X_{r}^{N}\left(i^{\prime} N^{-2 / 3}\right)\right)\right)^{j}}{j !(2 \sqrt{N})^{j}}\right) \wedge \bar{R}\right] .
\end{aligned}
$$

In order to determine the limit of the latter expectation, we first analyze the random variable inside the expectation. Applying Proposition 4.5 to the random walk bridges $X_{1}^{N}, X_{2}^{N}, \ldots, X_{R}^{N}$ and writing $B_{1}, B_{2}, \ldots, B_{R}$ for the Brownian bridges arising in the limit we find

$$
\begin{aligned}
& \prod_{i=1}^{T_{N} N^{2 / 3}} \frac{\sqrt{X_{r}^{N}\left(i N^{-2 / 3}\right) \wedge X_{r}^{N}\left((i-1) N^{-2 / 3}\right)}}{\sqrt{N}} \\
& \quad=\exp \left(-\frac{1}{2 N^{2 / 3}} \sum_{i=0}^{T_{N} N^{2 / 3}} N^{-1 / 3}\left(N-X_{r}^{N}\left(i N^{-2 / 3}\right)\right.\right.
\end{aligned}
$$




$$
\begin{aligned}
& \left.\left.\wedge X_{r}^{N}\left((i-1) N^{-2 / 3}\right)\right)+o(1)\right) \\
= & \exp \left(-\frac{1}{2} \int_{0}^{T} B_{r}(t) \mathrm{d} t+o(1)\right), \quad r=1,2, \ldots, R .
\end{aligned}
$$

Next, we employ the uniform convergence of $N^{-1 / 3}\left(N-X_{r}^{N}\right), r=1, \ldots, R$ (i.e., Proposition 4.5) to obtain

$$
\begin{aligned}
& \frac{\xi\left(X_{r}^{N}\left(i N^{-2 / 3}\right) \wedge X_{r}^{N}\left((i-1) N^{-2 / 3}\right)\right)}{\sqrt{X_{r}^{N}\left(i N^{-2 / 3}\right) \wedge X_{r}^{N}\left((i-1) N^{-2 / 3}\right)}} \\
& =\frac{\xi\left(X_{r}^{N}\left(i N^{-2 / 3}\right) \wedge X_{r}^{N}\left((i-1) N^{-2 / 3}\right)\right)}{\sqrt{N}} \\
& \cdot\left(1+O\left(N^{-2 / 3}\right)\right)
\end{aligned}
$$

for all $r=1,2, \ldots, R$. In addition, we note that there exists a real random variable $\mathcal{C}_{\xi}>0$ such that, with probability one,

$$
\max _{1 \leq m \leq N} \xi(m) \leq \mathcal{C}_{\xi} \log N, \quad N \in \mathbb{N} .
$$

Indeed, for a nonrandom $C \in \mathbb{R}$, the probability $\mathbb{P}\left(\max _{1 \leq m \leq N} \xi(m)>C \log N\right)$ can be written in terms of the tail distribution functions of the $\xi(m)$ 's which, in turn, can be estimated using (4.24) and Assumption 2.1(a). A simple application of the Borel-Cantelli lemma then gives (4.56). Putting together (4.55), (4.56) and the elementary inequalities $e^{z-z^{2}} \leq 1+z \leq e^{z}$ valid for all $z$ 's close enough to 0 we conclude that, for each $r=1,2, \ldots, R$, the product

$$
\prod_{i=1}^{T_{N} N^{2 / 3}}\left(1+\frac{\xi\left(X_{r}^{N}\left(i N^{-2 / 3}\right) \wedge X_{r}^{N}\left((i-1) N^{-2 / 3}\right)\right)}{\sqrt{X_{r}^{N}\left(i N^{-2 / 3}\right) \wedge X_{r}^{N}\left((i-1) N^{-2 / 3}\right)}}\right)
$$

behaves asymptotically as

$$
\exp \left(\sum_{i=1}^{T_{N} N^{2 / 3}} \frac{\xi\left(X_{r}^{N}\left(i N^{-2 / 3}\right) \wedge X_{r}^{N}\left((i-1) N^{-2 / 3}\right)\right)}{\sqrt{N}}\right),
$$

with an asymptotic multiplicative error term of at most

$$
\exp \left(\sum_{i=1}^{T_{N} N^{2 / 3}} \frac{\xi\left(X_{r}^{N}\left(i N^{-2 / 3}\right) \wedge X_{r}^{N}\left((i-1) N^{-2 / 3}\right)\right)^{2}}{N}\right)
$$

Moreover, an estimate on the fourth moment of the exponent in the latter exponential via Assumption 2.1(c) and the Borel-Cantelli lemma reveal the almost sure 
convergence of that exponent to 0. Consequently, the expression in (4.59) tends to 1 almost surely. Applying Proposition 4.5 to the exponent in (4.58) and combining the result with (4.54), we end up with the asymptotic

$$
\exp \left(-\frac{1}{2} \int_{0}^{T} B_{r}(t) \mathrm{d} t+s \xi \int_{0}^{\infty} L_{a}\left(B_{r}\right) \mathrm{d} W_{\xi}(a)+o(1)\right)
$$

for the second line in (4.53), for any $r=1,2, \ldots, R$.

Next, we apply Lemma 4.2 to $\Xi\left(X_{r}^{N}(0), X_{r}^{N}\left(T_{N}\right) ; N, T_{N}\right)$ in the first line of (4.53), use Proposition 4.5 for the third line of (4.53), and combine the results with (4.60) to find that the random variable inside the expectation in (4.53) converges in distribution to

$$
\begin{aligned}
\left(\prod_{r=1}^{R}\right. & \sqrt{\frac{2}{\pi T}} e^{-\left(B_{r}(0)-B_{r}(T)\right)^{2} /(2 T)} \mathbf{1}_{\left\{\forall t: B_{r}(t) \geq 0\right\}} \\
\cdot & \underline{R} \vee \exp \left(-\frac{1}{2} \int_{0}^{T} B_{r}(t) \mathrm{d} t+s \xi \int_{0}^{\infty} L_{a}\left(B_{r}\right) \mathrm{d} W_{\xi}(a)\right) \\
& \left.\frac{\left(s_{\mathfrak{a}} \int_{0}^{\infty} L_{a}\left(B_{r}\right) \mathrm{d} W_{\mathfrak{a}}(a)\right)^{j}}{j ! 2^{j}}\right) \wedge \bar{R} .
\end{aligned}
$$

Since the random variables in consideration are uniformly bounded, the expectation in (4.53) converges to the expectation of the random variable in (4.61). By Fubini's theorem, the latter is precisely the $R$ th moment of the limit in (4.47). Thanks to the boundedness of the random variables involved the convergence of moments also yields the convergence in distribution.

The joint convergence for several $T$ 's, $f$ 's and $g$ 's can be established in exactly the same way, by considering joint moments. Finally, the convergences in (4.49) are direct consequences of (4.38), (4.39) in Proposition 4.5, and the joint convergence with (4.47) can be deduced by taking joint moments with bounded continuous functionals of the prelimit expressions in (4.49).

LEMma 4.4. The convergences in Proposition 4.6 hold in the sense of moments for any $K \in[0, \infty), \underline{R} \in[-\infty, 0], \bar{R} \in[0, \infty]$.

PROOF. Our proof for the convergence in distribution of the random variable inside the expectation in (4.53) to the one in (4.61) remains valid when one of $\underline{R}, \bar{R}$ or both of them are infinite. It therefore remains to show the convergence of the corresponding expectations. To this end, it suffices to establish the uniform integrability of the prelimit random variables which, in turn, would follow from the uniform boundedness of their second moments. 
Clearly, for any $\underline{R} \in[-\infty, 0], \bar{R} \in[0, \infty]$, the second moment of the random variable inside the expectation in (4.53) is bounded above by

$$
\begin{aligned}
\mathbb{E}\left[\prod_{r=1}^{R} \Xi\left(X_{r}^{N}(0), X_{r}^{N}\left(T_{N}\right) ; N, T_{N}\right)^{2}\right. \\
\quad \cdot \prod_{i=1}^{T_{N} N^{2 / 3}\left(1+\frac{\xi\left(X_{r}^{N}\left(i N^{-2 / 3}\right) \wedge X_{r}^{N}\left((i-1) N^{-2 / 3}\right)\right)}{\sqrt{X_{r}^{N}\left(i N^{-2 / 3}\right) \wedge X_{r}^{N}\left((i-1) N^{-2 / 3}\right)}}\right)^{2}} \\
\left.\quad \cdot \frac{\left(\sum_{i^{\prime}=0}^{T_{N} N^{2 / 3}} \mathfrak{a}\left(X_{r}^{N}\left(i^{\prime} N^{-2 / 3}\right)\right)\right)^{2 j}}{(j !)^{2}(2 \sqrt{N})^{2 j}}\right] .
\end{aligned}
$$

In view of Lemma 4.2, we can bound the factors $\Xi\left(X_{r}^{N}(0), X_{r}^{N}\left(T_{N}\right) ; N, T_{N}\right)^{2}$, $r=1,2, \ldots, R$ by a uniform constant. Moreover, we can estimate the expectation with respect to the $\xi$ 's of the second product in the first line of (4.62) by applying

$$
X_{r}^{N}\left(i N^{-2 / 3}\right) \wedge X_{r}^{N}\left((i-1) N^{-2 / 3}\right) \geq\left\lfloor N-N^{1 / 3} K\right\rfloor-T_{N} N^{2 / 3} \geq N / 2
$$

to the denominators for all large enough $N$ and then using (4.25). Finally, to the quantity in the second line of (4.62) we apply the chain of elementary inequalities

$$
\left(\frac{|z|^{j}}{j !}\right)^{2} \leq e^{2|z|} \leq e^{2 z}+e^{-2 z}, \quad z \in \mathbb{R}, j \in \mathbb{N} \cup\{0\}
$$

and then bound the expectation with respect to the a's by means of (4.24). All in all, we end up with the estimate

$$
\begin{aligned}
C \mathbb{E}\left[\operatorname { e x p } \left(C \sum _ { h \in N ^ { - 1 / 3 } ( \mathbb { Z } + 1 / 2 ) } \left(\left|\mathbb{E}\left[\xi\left(N-N^{1 / 3} h-1 / 2\right)\right]\right| \frac{\sum_{r=1}^{R} L_{h}\left(X_{r}^{N}\right)}{N^{1 / 6}}\right.\right.\right. \\
\left.\quad+\frac{\sum_{r=1}^{R} L_{h}\left(X_{r}^{N}\right)^{2}}{N^{1 / 3}}+\frac{\sum_{r=1}^{R} L_{h}\left(X_{r}^{N}\right)^{\gamma^{\prime}}}{N^{\prime} / 6}\right) \\
+C \sum_{h \in N^{-1 / 3} \mathbb{Z}}\left(\left|\mathbb{E}\left[\mathfrak{a}\left(N-N^{1 / 3} h\right)\right]\right|\right. \\
\left.\left.\left.\quad \cdot \frac{\sum_{r=1}^{R} L_{h}\left(X_{r}^{N}\right)}{N^{1 / 6}}+\frac{\sum_{r=1}^{R} L_{h}\left(X_{r}^{N}\right)^{2}}{N^{1 / 3}}+\frac{\sum_{r=1}^{R} L_{h}\left(X_{r}^{N}\right)^{\gamma^{\prime}}}{N^{\prime} / 6}\right)\right)\right]
\end{aligned}
$$

where $C>0$ is a uniform constant and $2<\gamma^{\prime}<3$ is as in Lemma 4.1.

Finally, we recall from Assumption 2.1 that $|\mathbb{E}[\xi(m)]|=o\left(m^{-1 / 3}\right)$ and $|\mathbb{E}(\mathfrak{a}(m))|=o\left(m^{-1 / 3}\right)$ and from above that the bridges $X_{r}^{N}, r=1,2, \ldots, R$ do 
not reach $N / 2$ for all large enough $N$. Consequently,

$$
\begin{aligned}
\sum_{h \in N^{-1 / 3}(\mathbb{Z}+1 / 2)}\left|\mathbb{E}\left[\xi\left(N-N^{1 / 3} h-1 / 2\right)\right]\right| \frac{\sum_{r=1}^{R} L_{h}\left(X_{r}^{N}\right)}{N^{1 / 6}} & =o\left(N^{-1 / 3} \frac{N^{1 / 3}}{N^{1 / 6}}\right), \\
\sum_{h \in N^{-1 / 3} \mathbb{Z}}\left|\mathbb{E}\left[\mathfrak{a}\left(N-N^{1 / 3} h\right)\right]\right| \frac{\sum_{r=1}^{R} L_{h}\left(X_{r}^{N}\right)}{N^{1 / 6}} & =o\left(N^{-1 / 3} \frac{N^{1 / 3}}{N^{1 / 6}}\right),
\end{aligned}
$$

where the error terms are nonrandom and tend to 0 in the limit $N \rightarrow \infty$. The remaining part of the expression in (4.63) can be controlled by applying the CauchySchwarz inequality and then (4.5) (recall Remark 4.1).

LEMMA 4.5. The convergences in Proposition 4.6 hold in distribution for any $K \in[0, \infty), \underline{R} \in[-\infty, 0], \bar{R} \in[0, \infty]$.

REMARK 4.3. Note that, for infinite $\underline{R}, \bar{R}$, one should expect the moments of the limits in (4.47), (4.48) to grow very fast, since the latter include exponentials of random variables with Gaussian tails. Therefore, a priori, the just established convergence of moments does not imply the convergence in distribution.

ProOF OF LEMMA 4.5. For finite $\underline{R}, \bar{R}$, the lemma is a direct consequence of Lemma 4.4 and the boundedness of the limits in (4.47), (4.48). We turn to the case that $\underline{R}$ is finite and $\bar{R}$ is infinite. As before, we only consider $\operatorname{Sc}^{(j)}(N ; K, \underline{R}, \infty)$ and functions $f \geq 0, g \geq 0$ (otherwise we can write $f, g$ as the differences of their positive and negative parts and use the joint convergence in distribution of the associated scalar products). The convergence of moments shows that the random variables $\operatorname{Sc}^{(j)}(N ; K, \underline{R}, \infty), N \in \mathbb{N}$ form a tight sequence, so it suffices to identify the limit points of the latter with the random variable in (4.47). Let $\operatorname{Sc}^{(j)}(\infty ; K, \underline{R}, \infty)$ be such a limit point.

For each $N \in \mathbb{N}$ and $\bar{R} \in[0, \infty)$, the random variable $\operatorname{Sc}^{(j)}(N ; K, \underline{R}, \infty)$ stochastically dominates $\operatorname{Sc}^{(j)}(N ; K, \underline{R}, \bar{R})$. Consequently, the limit point $\mathrm{Sc}^{(j)}(\infty ; K, \underline{R}, \infty)$ must stochastically dominate the limit in distribution $\lim _{N \rightarrow \infty} \operatorname{Sc}^{(j)}(N ; K, \underline{R}, \bar{R})$ for every $\bar{R} \in[0, \infty)$. The latter is given by the expression in (4.47) and, by the monotone convergence theorem, tends in the limit $\bar{R} \uparrow \infty$ to the corresponding expression with $\bar{R}=\infty$. We conclude that the limit point $\mathrm{Sc}^{(j)}(\infty ; K, \underline{R}, \infty)$ and the expression in (4.47) with $\bar{R}=\infty$ are two nonnegative random variables with equal moments such that the first of them stochastically dominates the second. Clearly, such random variables must have the same distribution.

Similarly, the case $\underline{R}=-\infty$ can be dealt with by using the stochastic domination as $\underline{R}$ varies. The same argument also applies to any finite collection of $j$ 's, $T$ 's and nonnegative $f$ 's, $g$ 's and gives the joint convergence with (4.49) as well. 
So far, we have established Proposition 4.6, which identifies the leading order contributions in the limits of Theorem 4.2. It remains to control the associated remainder terms. To this end, for each $N \in \mathbb{N}$ and $K \in[0, \infty)$, we define

$$
\begin{aligned}
& \overline{\operatorname{Sc}}^{(j)}(N ; K)=\operatorname{Sc}^{(j)}\left(N ; N^{2 / 3},-\infty, \infty\right)-\operatorname{Sc}^{(j)}(N ; K,-\infty, \infty), \\
& \overline{\operatorname{Tr}}^{(j)}(N ; K)=\operatorname{Tr}^{(j)}\left(N ; N^{2 / 3},-\infty, \infty\right)-\operatorname{Tr}^{(j)}(N ; K,-\infty, \infty) .
\end{aligned}
$$

Lemma 4.6. There exist positive constants $C(R, K), R \in \mathbb{N}, K \in[0, \infty)$ (possibly depending on $f, g$, but not on $N$ ) such that

$$
\mathbb{E}\left[\left|\overline{\mathrm{Sc}}^{(j)}(N ; K)\right|^{R}\right] \leq \frac{C(R, K)}{2^{j R}}, \quad \mathbb{E}\left[\left|\overline{\operatorname{Tr}}^{(j)}(N ; K)\right|^{R}\right] \leq \frac{C(R, K)}{2^{j R}}
$$

for all $N \in \mathbb{N}$, and $\lim _{K \rightarrow \infty} C(R, K)=0, R \in \mathbb{N}$.

PROOF. We only give the proof for $\overline{\mathrm{Sc}}^{(j)}(N ; K)$, since the argument for $\overline{\operatorname{Tr}}^{(j)}(N ; K)$ is very similar. With $\mathcal{R}_{N, K}:=\left[0, N^{2 / 3}\right]^{2} \backslash[0, K]^{2}$ and the notation introduced in the proof of Lemma 4.3, the $R$ th absolute moment of $\overline{\mathrm{Sc}}^{(j)}(N ; K)$ is bounded above by

$$
\begin{aligned}
\mathbb{E}\left[\int _ { ( \mathcal { R } _ { N , K } ) ^ { R } } \prod _ { r = 1 } ^ { R } \left(\left|f\left(x_{r}\right)\right|\left|g\left(y_{r}\right)\right| \Xi\left(x_{r}, y_{r} ; N, T_{N}\right)\right.\right. \\
\cdot \mathbb{E}_{X_{r}^{N}}\left[\prod_{i=1}^{T_{N} N^{2 / 3}} \frac{\sqrt{X_{r}^{N}\left(i N^{-2 / 3}\right) \wedge X_{r}^{N}\left((i-1) N^{-2 / 3}\right)}}{\sqrt{N}}\right. \\
\cdot\left|1+\frac{\xi\left(X_{r}^{N}\left(i N^{-2 / 3}\right) \wedge X\left((i-1) N^{-2 / 3}\right)\right)}{\sqrt{X_{r}^{N}\left(i N^{-2 / 3}\right) \wedge X_{r}^{N}\left((i-1) N^{-2 / 3}\right)}}\right| \\
\left.\left.\left.\cdot \frac{\left|\sum_{i^{\prime}=0}^{T_{N} N^{2 / 3}} \mathfrak{a}\left(X_{r}^{N}\left(i^{\prime} N^{-2 / 3}\right)\right)\right|^{j}}{j !(2 \sqrt{N})^{j}}\right] \mathrm{~d} x_{r} \mathrm{~d} y_{r}\right)\right] .
\end{aligned}
$$

Relying on Fubini's theorem, we can first take all expectations in (4.64) and only then integrate over $\left(\mathcal{R}_{N, K}\right)^{R}$. Moreover, for fixed $x_{r}, y_{r}, r=1,2, \ldots, R$, we can use Hölder's inequality to bound the expectations inside the integral over $\left(\mathcal{R}_{N, K}\right)^{R}$ by the product of the $R$ th roots of the expectations

$$
\begin{aligned}
\mathbb{E}\left[\left|f\left(x_{r}\right)\right|^{R}\left|g\left(y_{r}\right)\right|^{R} \Xi\left(x_{r}, y_{r} ; N, T_{N}\right)^{R}\right. \\
\quad \cdot \prod_{i=1}^{T_{N} N^{\frac{2}{3}}}\left(\frac{X_{r}^{N}\left(i N^{-\frac{2}{3}}\right) \wedge X_{r}^{N}\left((i-1) N^{-\frac{2}{3}}\right)}{N}\right)^{\frac{R}{2}}
\end{aligned}
$$




$$
\begin{gathered}
\cdot\left|1+\frac{\xi\left(X_{r}^{N}\left(i N^{-2 / 3}\right) \wedge X_{r}^{N}\left((i-1) N^{-2 / 3}\right)\right)}{\sqrt{X_{r}^{N}\left(i N^{-2 / 3}\right) \wedge X_{r}^{N}\left((i-1) N^{-2 / 3}\right)}}\right|^{R} \\
\left.. \frac{\left|\sum_{i^{\prime}=0}^{T_{N} N^{2 / 3}} \mathfrak{a}\left(X_{r}^{N}\left(i^{\prime} N^{-2 / 3}\right)\right)\right|^{j R}}{(j !)^{R}(2 \sqrt{N})^{j R}}\right]
\end{gathered}
$$

$r=1,2, \ldots, R$.

Next, we recall that $f(x)=O\left(\exp \left(x^{1-\delta}\right)\right), g(x)=O\left(\exp \left(x^{1-\delta}\right)\right)$ as $x \rightarrow \infty$ for some $\delta>0$ by assumption. With that $\delta$ and any fixed $\epsilon \in\left(0, \frac{2 \delta}{3}\right)$, we first consider the case that $x_{r}, y_{r}$ in (4.65) are both at most $N^{2 / 3-\varepsilon}$. In this case, we estimate $\Xi\left(x_{r}, y_{r} ; N, T_{N}\right)$ using the inequality (4.51) of Lemma 4.2, bound the expectation with respect to the $\xi$ 's and $\mathfrak{a}$ 's as in the derivation of (4.63) in the proof of Lemma 4.4, and employ Assumption 2.1(a). All in all, we obtain the following upper bound on the expectation in (4.65):

$$
\frac{C}{2^{j R}}\left|f\left(x_{r}\right)\right|^{R}\left|g\left(y_{r}\right)\right|^{R} e^{-\frac{R}{C}\left(x_{r}-y_{r}\right)^{2}}
$$

$$
\begin{aligned}
& \cdot \mathbb{E}\left[\operatorname { e x p } \left(-\frac{R}{2 N} \sum_{i=1}^{T_{N} N^{\frac{2}{3}}}\left(N-X_{r}^{N}\left(i N^{-\frac{2}{3}}\right)\right)\right.\right. \\
+ & C \sum_{h \in N^{-1 / 3}(\mathbb{Z}+1 / 2)}\left(N^{-1 / 3} \frac{R L_{h}\left(X_{r}^{N}\right)}{N^{1 / 6}}+\frac{R^{2} L_{h}\left(X_{r}^{N}\right)^{2}}{N^{1 / 3}}+\frac{R^{\gamma^{\prime}} L_{h}\left(X_{r}^{N}\right)^{\gamma^{\prime}}}{N^{\gamma^{\prime} / 6}}\right) \\
+ & \left.\left.C \sum_{h \in N^{-1 / 3} \mathbb{Z}}\left(N^{-1 / 3} \frac{R L_{h}\left(X_{r}^{N}\right)}{N^{1 / 6}}+\frac{R^{2} L_{h}\left(X_{r}^{N}\right)^{2}}{N^{1 / 3}}+\frac{R^{\gamma^{\prime}} L_{h}\left(X_{r}^{N}\right)^{\gamma^{\prime}}}{N^{\gamma^{\prime} / 6}}\right)\right)\right],
\end{aligned}
$$

where $C>0$ is a uniform constant and $2 \leq \gamma^{\prime}<3$ is the same as in Lemma 4.1. An application of the Cauchy-Schwarz inequality to the latter expectation results further in the two factors

$$
\begin{aligned}
& \mathbb{E}\left[\exp \left(-\frac{R}{N} \sum_{i=1}^{T_{N} N^{2 / 3}}\left(N-X_{r}^{N}\left(i N^{-2 / 3}\right)\right)\right)\right]^{1 / 2}, \\
& \mathbb{E}\left[\operatorname { e x p } \left(2 C \sum_{h \in N^{-1 / 3}(\mathbb{Z}+1 / 2)}\left(\frac{R L_{h}\left(X_{r}^{N}\right)}{N^{1 / 2}}+\frac{R^{2} L_{h}\left(X_{r}^{N}\right)^{2}}{N^{1 / 3}}+\frac{R^{\gamma^{\prime}} L_{h}\left(X_{r}^{N}\right)^{\gamma^{\prime}}}{N^{\gamma^{\prime} / 6}}\right)\right.\right. \\
& \left.\left.\quad+2 C \sum_{h \in N^{-1 / 3} \mathbb{Z}}\left(\frac{R L_{h}\left(X_{r}^{N}\right)}{N^{1 / 2}}+\frac{R^{2} L_{h}\left(X_{r}^{N}\right)^{2}}{N^{1 / 3}}+\frac{R^{\gamma^{\prime}} L_{h}\left(X_{r}^{N}\right)^{\gamma^{\prime}}}{N^{\gamma^{\prime} / 6}}\right)\right)\right]^{1 / 2}
\end{aligned}
$$

To estimate the expression in (4.67) further, we assume first that $x_{r} \leq y_{r}$. In this case, the random walk bridge $X_{r}^{N}$ can be sampled as follows: sample a random walk bridge from $N$ to $N$ in $T_{N} N^{2 / 3}-\left\lfloor N-N^{1 / 3} x_{r}\right\rfloor+\left\lfloor N-N^{1 / 3} y_{r}\right\rfloor$ steps of size \pm 1 ; next, shift the resulting bridge down by $N-\left\lfloor N-N^{1 / 3} x_{r}\right\rfloor$; finally, insert 
$\left\lfloor N-N^{1 / 3} x_{r}\right\rfloor-\left\lfloor N-N^{1 / 3} y_{r}\right\rfloor$ additional down steps of size -1 uniformly at random. Combining the observation that the latter insertion is only increasing the value of $\sum_{i=1}^{T_{N} N^{2 / 3}}\left(N-X_{r}^{N}\left(i N^{-2 / 3}\right)\right)$ with Proposition 4.2 for the random walk bridge connecting $N$ to $N$ we obtain the bound

$$
\mathbb{E}\left[\exp \left(-\frac{R}{N} \sum_{i=1}^{T_{N} N^{2 / 3}}\left(N-X_{r}^{N}\left(i N^{-2 / 3}\right)\right)\right)\right]^{1 / 2} \leq \tilde{C} e^{-R T_{N} x_{r} / 2}
$$

where $\tilde{C}>0$ is a constant depending only on $R$. A similar argument in the case $x_{r}>y_{r}$ leads to the same upper bound, only with $x_{r}$ replaced by $y_{r}$.

We turn to the expression in (4.68). Our goal is to give bounds on the exponential moments of the six random variables

$$
\begin{aligned}
& \sum_{h \in N^{-1 / 3}(\mathbb{Z}+1 / 2)} \frac{L_{h}\left(X_{r}^{N}\right)}{N^{1 / 2}}, \quad \sum_{h \in N^{-1 / 3}(\mathbb{Z}+1 / 2)} \frac{L_{h}\left(X_{r}^{N}\right)^{2}}{N^{1 / 3}}, \\
& \sum_{h \in N^{-1 / 3}(\mathbb{Z}+1 / 2)} \frac{L_{h}\left(X_{r}^{N}\right)^{\gamma^{\prime}}}{N \gamma^{\prime} / 6}, \quad \sum_{h \in N^{-1 / 3} \mathbb{Z}} \frac{L_{h}\left(X_{r}^{N}\right)}{N^{1 / 2}}, \\
& \sum_{h \in N^{-1 / 3} \mathbb{Z}} \frac{L_{h}\left(X_{r}^{N}\right)^{2}}{N^{1 / 3}}, \quad \sum_{h \in N^{-1 / 3} \mathbb{Z}} \frac{L_{h}\left(X_{r}^{N}\right)^{\gamma^{\prime}}}{N \gamma^{\prime} / 6},
\end{aligned}
$$

which can be then combined by means of Hölder's inequality. The first and fourth random variables simply equal to $T_{N} N^{-1 / 6}$ and $T_{N} N^{-1 / 6}+N^{-5 / 6}$, respectively. For the other four random variables in (4.69), we use the estimate (4.19) [recalling also Remark 4.1 and the elementary inequality (4.15)] to obtain

$$
\begin{aligned}
& \max \left(\sum_{h \in N^{-1 / 3}(\mathbb{Z}+1 / 2)} \frac{L_{h}\left(X_{r}^{N}\right)^{p}}{N^{1 / 3}}, \sum_{h \in N^{-1 / 3} \mathbb{Z}} \frac{L_{h}\left(X_{r}^{N}\right)^{p}}{N^{1 / 3}}\right) \\
& \quad \leq C\left(M\left(N, T_{N}\right)^{p-1}+\left|x_{r}-y_{r}\right|^{p-1}+1\right),
\end{aligned}
$$

where $p \in\left\{2, \gamma^{\prime}\right\}, C>0$ is a uniform constant, and $N^{1 / 3} M\left(N, T_{N}\right)$ has the same law as the difference between the maximum and the minimum of $X_{r}^{N}$ [see the explanation following (4.19)]. The arguing as in the proof of Proposition 4.2, we conclude that $N^{1 / 3} M\left(N, T_{N}\right)$ is stochastically dominated by the sum of $\left|x_{r}-y_{r}\right|$ and the difference between the maximum and the minimum of a simple symmetric random walk going from 0 to 0 in $T_{N} N^{2 / 3}$ steps of size \pm 1 . At this point, the bound (4.7) shows that

$$
\begin{aligned}
& \mathbb{E}\left[\exp \left(\theta \sum_{h \in N^{-1 / 3}(\mathbb{Z}+1 / 2)} \frac{L_{h}\left(X_{r}^{N}\right)^{p}}{N^{1 / 3}}\right)\right] \vee \mathbb{E}\left[\exp \left(\theta \sum_{h \in N^{-1 / 3} \mathbb{Z}} \frac{L_{h}\left(X_{r}^{N}\right)^{p}}{N^{1 / 3}}\right)\right] \\
& \leq \tilde{C} e^{\tilde{C}\left|x_{r}-y_{r}\right|^{p-1}},
\end{aligned}
$$

where $\theta>0, p \in\left\{2, \gamma^{\prime}\right\}$, and $\tilde{C}>0$ is a constant depending only on $\theta$. 
Putting everything together, we get the following estimate on the expression in (4.66):

$$
\begin{aligned}
& \frac{\tilde{C}}{2^{j R}}\left|f\left(x_{r}\right)\right|^{R}\left|g\left(y_{r}\right)\right|^{R} \\
& \quad \exp \left(-\frac{R}{C}\left(x_{r}-y_{r}\right)^{2}-\frac{R T_{N}}{2} \min \left(x_{r}, y_{r}\right)\right. \\
& \left.\quad+\tilde{C}\left|x_{r}-y_{r}\right|+\tilde{C}\left|x_{r}-y_{r}\right|^{\gamma^{\prime}-1}\right)
\end{aligned}
$$

where $\tilde{C}>0$ is a constant depending only on $R$, and $C>0$ is a uniform constant. Since $f(x)=O\left(\exp \left(x^{1-\delta}\right)\right), g(x)=O\left(\exp \left(x^{1-\delta}\right)\right)$ as $x \rightarrow \infty$, and $2<\gamma^{\prime}<3$, the integral of the $R$ th root of the latter expression [and hence, also of the expression in (4.65)] over $\left\{\left(x_{r}, y_{r}\right) \in \mathcal{R}_{N, K}: x_{r}, y_{r} \leq N^{2 / 3-\epsilon}\right\}$ admits an upper bound of the form $\frac{C(R, K)}{2^{j}}$ with $\lim _{K \rightarrow \infty} C(R, K)=0$ for any fixed $R$.

To complete the proof, it remains to treat the case of at least one of $x_{r}, y_{r}$ is larger than $N^{2 / 3-\epsilon}$. The additional technical difficulty in this case is that the quantities $\sqrt{X_{r}^{N}\left(i N^{-2 / 3}\right) \wedge X_{r}^{N}\left((i-1) N^{-2 / 3}\right)}$ can be anywhere between 1 and $\sqrt{N-N^{1-\epsilon}+T_{N} N^{2 / 3}} \leq \sqrt{N-N^{1-\epsilon} / 2}$ for all $N$ sufficiently large. To address this, we bound the factors involving $X_{r}^{N}\left(i N^{-2 / 3}\right) \wedge X_{r}^{N}\left((i-1) N^{-2 / 3}\right)$ in (4.65) as follows:

$$
\begin{aligned}
N^{-R / 2} \mid & \sqrt{X_{r}^{N}\left(i N^{-2 / 3}\right) \wedge X_{r}^{N}\left((i-1) N^{-2 / 3}\right)} \\
& +\left.\xi\left(X_{r}^{N}\left(i N^{-2 / 3}\right) \wedge X_{r}^{N}\left((i-1) N^{-2 / 3}\right)\right)\right|^{R} \\
\leq & N^{-R / 2}\left(\sqrt{N-N^{1-\varepsilon} / 2}+\left|\xi\left(X_{r}^{N}\left(i N^{-2 / 3}\right) \wedge X_{r}^{N}\left((i-1) N^{-2 / 3}\right)\right)\right|\right)^{R} \\
= & \left(1-N^{-\varepsilon} / 2\right)^{R / 2}\left(1+\frac{\left|\xi\left(X_{r}^{N}\left(i N^{-2 / 3}\right) \wedge X_{r}^{N}\left((i-1) N^{-2 / 3}\right)\right)\right|}{\sqrt{N-N^{1-\varepsilon} / 2}}\right)^{R} .
\end{aligned}
$$

Inserting the latter bound into (4.65) and proceeding as in the derivation of (4.66) (only replacing all $\xi$ 's by their absolute values), we obtain an estimate on the expression in (4.65) of the form

$$
\begin{aligned}
\frac{C}{2^{j R}}\left|f\left(x_{r}\right)\right|^{R}\left|g\left(y_{r}\right)\right|^{R} \exp \left(-\frac{R}{C}\left(x_{r}-y_{r}\right)^{2}-\frac{R T_{N}}{4} N^{2 / 3-\epsilon}\right) \\
\cdot \mathbb{E}\left[\operatorname { e x p } \left(C \sum_{h \in N^{-1 / 3}(\mathbb{Z}+1 / 2)}\left(\frac{R L_{h}\left(X_{r}^{N}\right)}{N^{1 / 6}}+\frac{R^{2} L_{h}\left(X_{r}^{N}\right)^{2}}{N^{1 / 3}}+\frac{R^{\gamma^{\prime}} L_{h}\left(X_{r}^{N}\right)}{N^{\gamma^{\prime} / 6}}\right)\right.\right. \\
\left.\left.+C \sum_{h \in N^{-1 / 3} \mathbb{Z}}\left(\frac{R L_{h}\left(X_{r}^{N}\right)}{N^{1 / 6}}+\frac{R^{2} L_{h}\left(X_{r}^{N}\right)^{2}}{N^{1 / 3}}+\frac{R^{\gamma^{\prime}} L_{h}\left(X_{r}^{N}\right)}{N^{\gamma^{\prime} / 6}}\right)\right)\right]
\end{aligned}
$$


where $C>0$ is a uniform constant and $2 \leq \gamma^{\prime}<3$ is the same as in Lemma 4.1. Repeating the argument leading to (4.70), we arrive at the upper bound

$$
\begin{aligned}
& \frac{\tilde{C}}{2^{j R}}\left|f\left(x_{r}\right)\right|^{R}\left|g\left(y_{r}\right)\right|^{R} \\
& \quad \exp \left(-\frac{R}{C}\left(x_{r}-y_{r}\right)^{2}-\frac{R T_{N}}{4} N^{2 / 3-\varepsilon}\right. \\
& \left.\quad+\tilde{C}\left(T_{N} N^{1 / 6}+\left|x_{r}-y_{r}\right|+\left|x_{r}-y_{r}\right|^{\gamma^{\prime}-1}\right)\right)
\end{aligned}
$$

with the constant $C$ of (4.71) and a constant $\tilde{C}>0$ depending only on $R$. Since $f(x)=O\left(\exp \left(x^{1-\delta}\right)\right), g(x)=O\left(\exp \left(x^{1-\delta}\right)\right)$ as $x \rightarrow \infty$ and $2<\gamma^{\prime}<3$, the term $\exp \left(-\frac{R}{C}\left(x_{r}-y_{r}\right)^{2}-\frac{R T_{N}}{4} N^{2 / 3-\epsilon}\right)$ dominates on the region of integration $\left\{\left(x_{r}, y_{r}\right) \in \mathcal{R}_{N, K}: x_{r}>N^{2 / 3-\epsilon}\right.$ or $\left.y_{r}>N^{2 / 3-\epsilon}\right\}$. The area of the latter is of the order $N^{4 / 3}$, so that the desired estimate readily follows.

REMARK 4.4. Lemma 4.6 shows that the differences between the integrals in (4.45), (4.46) with $\underline{R}=-\infty, \bar{R}=\infty$ and a fixed $K$ and those with $\underline{R}=-\infty$, $\bar{R}=\infty$ and $K=N^{2 / 3}$ are bounded uniformly in $N \in \mathbb{N}$ and tend to 0 in the limit $K \rightarrow \infty$ in the sense of moments. This statement is also true for the sums of such differences over $j=0,1, \ldots$ (which give rise to exponentials), since the bounds of Lemma 4.6 decay exponentially in $j$.

Next, we present an extension of Lemma 4.6 which can be shown in the same way.

LEMMA 4.7. Fix an $N \in \mathbb{N}$ and let $\Upsilon$ be a random variable given by a deterministic function of the random walk bridge $X$ and the sequence of $\mathfrak{a}$ 's. For each $K \in[0, \infty)$, define $\overline{\mathrm{Sc}}^{(j)}(N ; K ; \Upsilon)$ as the modification of $\overline{\mathrm{Sc}}^{(j)}(N ; K)$ obtained by inserting $\Upsilon$ as an additional factor into the expectation in (4.45). Suppose that the $(2 R)$ th moment of $\Upsilon$ is bounded by $C_{1}<\infty$. Then there exist positive constants $C(R, K), K \in[0, \infty)$ (possibly depending on $f, g$, but not on $N, \Upsilon$ ) such that

$$
\begin{aligned}
& \mathbb{E}\left[\left|\overline{\operatorname{Sc}}^{(j)}(N ; K ; \Upsilon)\right|^{R}\right] \leq \frac{C(R, K)}{2^{j R}} C_{1}^{1 / 2}, \\
& \mathbb{E}\left[\left|\overline{\operatorname{Tr}}^{(j)}(N ; K ; \Upsilon)\right|^{R}\right] \leq \frac{C(R, K)}{2^{j R}} C_{1}^{1 / 2}
\end{aligned}
$$

for all $N \in \mathbb{N}$, and $\lim _{K \rightarrow \infty} C(R, K)=0$.

As a last ingredient for the proof of Theorem 4.2, we need to control the error in replacing the terms involving $\mathfrak{a}$ 's in the fourth lines of (4.40), (4.41) by the terms involving $\mathfrak{a}$ 's in the third lines of (4.45), (4.46). To this end, we consider the 
complete homogeneous symmetric functions and power sums given respectively by

$$
\begin{aligned}
& h(j ; N):=\sum_{0 \leq i_{1} \leq \cdots \leq i_{j} \leq T_{N} N^{2 / 3}} \prod_{j^{\prime}=1}^{j} \mathfrak{a}\left(X\left(i_{j^{\prime}} N^{-2 / 3}\right)\right), \\
& p(j ; N):=\sum_{i^{\prime}=0}^{T_{N} N^{2 / 3}} \mathfrak{a}\left(X\left(i^{\prime} N^{-2 / 3}\right)\right)^{j} .
\end{aligned}
$$

The $h(j ; N)$ 's can be expressed in terms of the $p(j ; N)$ 's according to the Newton identities. The latter can be written as (see, e.g., [48], Chapter 1, Section 2):

$$
h(j ; N)=\left[z^{j}\right] \exp \left(\sum_{j^{\prime}=1}^{\infty} \frac{p\left(j^{\prime} ; N\right)}{j^{\prime}} z^{j^{\prime}}\right),
$$

where $\left[z^{j}\right]$ stands for the coefficient of $z^{j}$ in the series expansion of what follows. We note further that (4.73) implies

$$
\frac{h(j ; N)}{(2 \sqrt{N})^{j}}=\sum_{l=0}^{j} \frac{p(1 ; N)^{l}}{l !(2 \sqrt{N})^{l}}\left(\left[z^{j-l}\right] \exp \left(\sum_{j^{\prime}=2}^{\infty} \frac{p\left(j^{\prime} ; N\right)}{j^{\prime}(2 \sqrt{N})^{j^{\prime}}} z^{j^{\prime}}\right)\right) .
$$

The $l=j$ term in (4.74) is precisely the one appearing in the third lines of (4.45), (4.46). The next lemma shows that the other terms become negligible as $N \rightarrow \infty$.

LEMMA 4.8. Under Assumption 2.1 and for each $R=1,2, \ldots$, there exist positive constants $\tilde{C}$, $\varepsilon$ such that

$$
\mathbb{E}\left[\left(\left[z^{l}\right] \exp \left(\sum_{j^{\prime}=2}^{\infty} \frac{\left|p\left(j^{\prime} ; N\right)\right|}{j^{\prime}(2 \sqrt{N})^{j^{\prime}}} z^{j^{\prime}}\right)\right)^{R}\right] \leq\left(\tilde{C} N^{-\varepsilon}\right)^{l R}
$$

for all $l=1,2, \ldots, T_{N} N^{2 / 3}$ and $N \in \mathbb{N}$.

Proof. We define $\Gamma_{j}=\sup _{0 \leq i_{1} \leq \cdots \leq i_{j}} \mathbb{E}\left[\prod_{j^{\prime}=1}^{j}\left|\mathfrak{a}\left(i_{j^{\prime}}\right)\right|\right]=\sup _{i \in \mathbb{N}} \mathbb{E}\left[|\mathfrak{a}(i)|^{j}\right]$, where the second equality is due to Hölder's inequality. By Assumption 2.1, $\Gamma_{j} \leq C^{j} j^{j \gamma}$ with universal constants $C>0$ and $0<\gamma<3 / 4$. We also write $k$ for $T_{N} N^{2 / 3}+1$. Now, expanding the random variable inside the expectation in (4.75) into a sum of monomials in the $\left|\mathfrak{a}\left(X\left(i^{\prime} N^{-2 / 3}\right)\right)\right|$ 's, bounding the expectations of the latter by $\Gamma_{l R}$, and then collecting the terms back we obtain the following upper bound on the left-hand side of (4.75):

$$
\frac{1}{(2 \sqrt{N})^{l R}} \Gamma_{l R}\left(\left[z^{l}\right] \exp \left(\sum_{j^{\prime}=2}^{\infty} \frac{k}{j^{\prime}} z^{j^{\prime}}\right)\right)^{R} .
$$


Using $l \leq k$, we deduce further

$$
\begin{aligned}
{\left[z^{l}\right] \exp \left(\sum_{j^{\prime}=2}^{\infty} \frac{k}{j^{\prime}} z^{j^{\prime}}\right) } & =\left[z^{l}\right]\left(\sum_{l^{\prime}=0}^{\lfloor l / 2\rfloor} \frac{\left(\sum_{j^{\prime}=2}^{l} \frac{k}{j^{\prime}} z^{j^{\prime}}\right)^{l^{\prime}}}{l^{\prime} !}\right) \\
& \leq \sum_{l^{\prime}=0}^{\lfloor l / 2\rfloor} \frac{\left(\sum_{j^{\prime}=2}^{l} \frac{k}{j^{\prime}}\right)^{l^{\prime}}}{l^{\prime} !} \leq \sum_{l^{\prime}=0}^{\lfloor l / 2\rfloor} \frac{(k \log l)^{l^{\prime}}}{l^{\prime} !} \\
& \leq(\lfloor l / 2\rfloor+1) \frac{(k \log l)^{\lfloor l / 2\rfloor}}{\lfloor l / 2\rfloor !} \leq C^{l}\left(\frac{k \log l}{l}\right)^{\lfloor l / 2\rfloor},
\end{aligned}
$$

where $C>0$ is a uniform constant. Putting everything together, we find that the left-hand side of (4.75) is at most

$$
\begin{aligned}
\tilde{C}^{l R} \frac{1}{(2 \sqrt{N})^{l R}} l^{l R \gamma}\left(\frac{N^{\frac{2}{3}} \log l}{l}\right)^{\lfloor l / 2\rfloor R} & \leq\left(\frac{\tilde{C} l^{2 \gamma-1} N^{\frac{2}{3}} \log l}{4 N}\right)^{l R / 2} \\
& \leq\left(\frac{\tilde{C} N^{\frac{4 \gamma}{3}} \log N^{\frac{2}{3}}}{4 N}\right)^{l R / 2},
\end{aligned}
$$

where $\tilde{C}$ is a constant depending only on $R, \gamma$, and $\sup _{N} T_{N}$ that varies from expression to expression. The lemma now follows from $\gamma<3 / 4$ (see Assumption 2.1).

4.3. Proof of Theorem 2.1. As discussed in Section 4.2, to prove Theorem 2.1 it suffices to establish Theorem 4.2. In the following, we only give the proof of the latter for $\operatorname{Sc}(N)$, since the same proof applies to $\operatorname{Tr}(N)$ as well. To start with, we note

$$
\operatorname{Sc}(N)=\sum_{j=0}^{\left\lfloor T N^{2 / 3}\right\rfloor} \operatorname{Sc}^{(j)}(N ; K,-\infty, \infty)+\sum_{j=0}^{\left\lfloor T N^{2 / 3}\right\rfloor} \overline{\operatorname{Sc}}^{(j)}(N ; K)
$$

$$
+\sum_{j=0}^{\left\lfloor T N^{2 / 3}\right\rfloor} \sum_{l=0}^{j-1} U(N ; j, l)
$$

where

$$
\begin{aligned}
U(N ; j, l):= & \frac{1}{2} \int_{0}^{\infty} \int_{0}^{\infty} f(x) g(y) \Xi\left(x, y ; N, T_{N}\right) \mathbb{E}_{X}\left[\mathbf{1}_{\{\forall t: X(t) \leq N\}}\right. \\
& \cdot \prod_{i=1}^{T_{N} N^{2 / 3}} \frac{\sqrt{X\left(i N^{-2 / 3}\right) \wedge X\left((i-1) N^{-2 / 3}\right)}}{\sqrt{N}} \\
& \cdot\left(1+\frac{\xi\left(X\left(i N^{-2 / 3}\right) \wedge X\left((i-1) N^{-2 / 3}\right)\right)}{\sqrt{X\left(i N^{-2 / 3}\right) \wedge X\left((i-1) N^{-2 / 3}\right)}}\right)
\end{aligned}
$$




$$
\begin{aligned}
& \cdot \frac{\left(\sum_{i^{\prime}=0}^{T_{N} N^{2 / 3}} \mathfrak{a}\left(X\left(i^{\prime} N^{-2 / 3}\right)\right)\right)^{l}}{l !(2 \sqrt{N})^{l}} \\
& \left.\cdot\left[z^{j-l}\right] \exp \left(\sum_{j^{\prime}=2}^{\infty} \frac{\sum_{i^{\prime}=0}^{T_{N} N^{2 / 3}} \mathfrak{a}\left(X\left(i^{\prime} N^{-2 / 3}\right)\right)^{j^{\prime}}}{j^{\prime}(2 \sqrt{N}) j^{j^{\prime}}} z^{j^{\prime}}\right)\right] \mathrm{d} x \mathrm{~d} y .
\end{aligned}
$$

Hereby, with a slight abuse of notation we have allowed the value of $T_{N}$ to change from term to term with $j, l$. However, this is not important, since the results of the previous subsection apply to all occurring values of $T_{N}$.

Now, we take the limits $N \rightarrow \infty, K \rightarrow \infty$ (in this particular order) of the righthand side in (4.77). The limits as $N \rightarrow \infty$ of the summands in the first sum in (4.77) have been identified in Proposition 4.6, so that by invoking the moment bounds of Lemma 4.6 with $K=0$ we conclude that their sum converges as $N \rightarrow$ $\infty$ to

$$
\begin{aligned}
& \frac{1}{\sqrt{2 \pi T}} \int_{0}^{K} \int_{0}^{K} f(x) g(y) \exp \left(-\frac{(x-y)^{2}}{2 T}\right) \mathbb{E}_{B^{x, y}}\left[\mathbf{1}_{\left\{B^{x, y}(t) \geq 0\right\}}\right. \\
& \left.\cdot \exp \left(-\frac{1}{2} \int_{0}^{T} B^{x, y}(t) \mathrm{d} t+\sqrt{s_{\xi}^{2}+s_{\mathfrak{a}}^{2} / 4} \int_{0}^{\infty} L_{a}\left(B^{x, y}\right) \mathrm{d} W(a)\right)\right] \mathrm{d} x \mathrm{~d} y
\end{aligned}
$$

in the sense of moments and in law, where $W=\frac{1}{\sqrt{s_{\xi}^{2}+s_{\mathfrak{a}}^{2} / 4}}\left(s_{\xi} W_{\xi}+s_{\mathfrak{a}} W_{\mathfrak{a}}\right)$. In view of Remark 4.4, the $K \rightarrow \infty$ limit of the expression in (4.79) in the sense of moments and in distribution is given by the same expression with $K$ replaced by $\infty$. The latter is precisely the limit in (4.42) [recall Assumption 2.1(b)].

Next, for every fixed $R \in \mathbb{N}$, we combine Lemma 4.6 with the triangle inequality for the $L^{R}$-norm to conclude that the $R$ th moment of the second sum in (4.77) can be bounded above by a constant $C(R, K)$ uniformly in $N \in \mathbb{N}$, which further satisfies $\lim _{K \rightarrow \infty} C(R, K)=0$. Consequently, the second sum in (4.77) vanishes in the double limit $N \rightarrow \infty, K \rightarrow \infty$ in the sense of moments and in distribution.

To control the third sum in (4.77), we apply Lemma 4.7 for $U(N ; j, l)$ and a fixed $R \in \mathbb{N}$ (note that in the case at hand the bound on the $R$ th moment of $\Upsilon$ required in Lemma 4.7 is precisely the content of Lemma 4.8) to get

$$
\mathbb{E}\left[U(N ; j, l)^{R}\right] \leq \frac{\tilde{C}_{1}}{2^{l R}}\left(\tilde{C}_{2} N^{-\varepsilon}\right)^{(j-l) R}, \quad j, l \in \mathbb{N}, j \geq l,
$$

where the positive constants $\tilde{C}_{1}, \tilde{C}_{2}$ and $\epsilon$ may depend on $R$. Combining this with the triangle inequality for the $L^{R}$-norm, we deduce that the $R$ th moment of the third sum in (4.77) tends to 0 in the limit $N \rightarrow \infty$. Since $R \in \mathbb{N}$ was arbitrary, the third sum in (4.77) must vanish as $N \rightarrow \infty$ both in the sense of moments and in distribution.

REMARK 4.5. Note that the terms in (4.41) with even $j$ (odd $j$, resp.) arise when we take even (odd, resp.) powers of the matrix $\frac{M_{N}}{2 \sqrt{N}}$. Since Proposition 4.6 
gives the joint asymptotics of such terms for any finite collection of $j$ 's, we can separate the even and the odd powers for the traces in Theorem 2.1. By doing so, we obtain the following results. Let $T_{N}, N \in \mathbb{N}$ and $\tilde{T}_{N}, N \in \mathbb{N}$ be two sequences of positive numbers such that $T_{N} N^{2 / 3}, N \in \mathbb{N}$ are even integers and $\tilde{T}_{N} N^{2 / 3}, N \in \mathbb{N}$ are odd integers. Suppose further that $\sup _{N}\left|T_{N}-T\right| N^{2 / 3}<\infty$ and $\sup _{N}\left|\tilde{T}_{N}-T\right| N^{2 / 3}<\infty$. Then, under the same assumptions and in the same sense as in Theorem 2.1 we have

$$
\begin{aligned}
\lim _{N \rightarrow \infty} \operatorname{Trace}( & \left.\frac{1}{2}\left(\frac{M_{N}}{2 \sqrt{N}}\right)^{T_{N} N^{2 / 3}}\right) \\
= & \frac{1}{\sqrt{2 \pi T}} \int_{0}^{\infty} \mathbb{E}_{B^{x, x}}\left[\mathbf { 1 } _ { \{ \forall t : B ^ { x , y } ( t ) \geq 0 \} } \operatorname { e x p } \left(-\frac{1}{2} \int_{0}^{T} B^{x, y}(t) \mathrm{d} t\right.\right. \\
& \left.+s_{\xi} \int_{0}^{\infty} L_{a}\left(B^{x, y}\right) \mathrm{d} W_{\xi}(a)\right) \\
& \left.\cdot \sum_{j \text { even }} \frac{\left(s_{\mathfrak{a}} \int_{0}^{\infty} L_{a}\left(B^{x, y}\right) \mathrm{d} W_{\mathfrak{a}}(a)\right)^{j}}{2^{j} j !}\right] \mathrm{d} x .
\end{aligned}
$$

Similarly,

$$
\begin{aligned}
\lim _{N \rightarrow \infty} \operatorname{Trace}( & \left(\frac{1}{2}\left(\frac{M_{N}}{2 \sqrt{N}}\right)^{\tilde{T}_{N} N^{2 / 3}}\right) \\
= & \frac{1}{\sqrt{2 \pi T}} \int_{0}^{\infty} \mathbb{E}_{B^{x, x}}\left[\mathbf { 1 } _ { \{ \forall t : B ^ { x , y } ( t ) \geq 0 \} } \cdot \operatorname { e x p } \left(-\frac{1}{2} \int_{0}^{T} B^{x, y}(t) \mathrm{d} t\right.\right. \\
& \left.+s \xi \int_{0}^{\infty} L_{a}\left(B^{x, y}\right) \mathrm{d} W_{\xi}(a)\right) \\
& \left.\cdot \sum_{j \text { odd }} \frac{\left(s_{\mathfrak{a}} \int_{0}^{\infty} L_{a}\left(B^{x, y}\right) \mathrm{d} W_{\mathfrak{a}}(a)\right)^{j}}{2^{j} j !}\right] \mathrm{d} x .
\end{aligned}
$$

Here, $W_{\xi}$ and $W_{\mathfrak{a}}$ are independent standard Brownian motions as in Proposition 4.6. Let us emphasize that we claim convergence only for the traces, but not for the operators themselves, as the latter is more delicate.

5. Properties of the stochastic Airy semigroup I. In this section, we study the properties of operators $\mathcal{U}(T), T>0$ in more detail. The present section contains the statements that we prove directly from the definition of $\mathcal{U}(T), T>0$, while in Sections 6, 7 we prove the remaining properties by relying on the asymptotic results of Section 4.

LEMMA 5.1. For each $T>0, \mathcal{U}(T)$ is a Hilbert-Schmidt operator on $L^{2}\left(\mathbb{R}_{\geq 0}\right)$ with probability one. 
ProOF. Fix a $T>0$. We need to prove that the integral kernel $K(x, y ; T)$ of $\mathcal{U}(T)$ satisfies

$$
\int_{\mathbb{R}_{\geq 0}} \int_{\mathbb{R}_{\geq 0}} K(x, y ; T)^{2} \mathrm{~d} x \mathrm{~d} y<\infty
$$

with probability one. To this end, we will show that the expectation of the latter double integral is finite. Moving the square inside the expectation in the definition of $K(x, y ; T)^{2}$, dropping the indicator function therein, applying Fubini's theorem to take the expectation with respect to $W$, and observing that the latter expectation boils down to the exponential moment of a Gaussian random variable, we obtain the following upper bound on the expectation of (5.1):

$$
\begin{aligned}
\frac{1}{2 \pi T} & \int_{\mathbb{R}_{\geq 0}} \int_{\mathbb{R}_{\geq 0}} e^{-\frac{(x-y)^{2}}{T}} \\
& \cdot \mathbb{E}_{B^{x, y}}\left[\exp \left(-\int_{0}^{T} B^{x, y}(t) \mathrm{d} t+\frac{2}{\beta} \int_{0}^{\infty} L_{a}\left(B^{x, y}\right)^{2} \mathrm{~d} a\right)\right] \mathrm{d} x \mathrm{~d} y .
\end{aligned}
$$

Next, we note that $B^{x, y}(t)-\left(1-\frac{t}{T}\right) x-\frac{t}{T} y, t \in[0, T]$ is a copy of $B^{0,0}$, as well as $\left(1-\frac{t}{T}\right) x+\frac{t}{T} y \geq \min (x, y)$. These and the Cauchy-Schwarz inequality allow to estimate the integrand in (5.2) by the product of $e^{-\frac{(x-y)^{2}}{T}-\min (x, y)}$ with

$$
\mathbb{E}_{B^{0,0}}\left[\exp \left(-2 \int_{0}^{T} B^{0,0}(t) \mathrm{d} t\right)\right]^{1 / 2} \mathbb{E}_{B^{x, y}}\left[\exp \left(\frac{4}{\beta} \int_{0}^{\infty} L_{a}\left(B^{x, y}\right)^{2} \mathrm{~d} a\right)\right]^{1 / 2}
$$

Further, it is well known that the first expectation in (5.3) is given by a finite constant $C$; see, e.g., [21], proof of Theorem 3.1, final display, for a significantly stronger statement. For the second expectation in (5.3), we recall from the proof of Proposition 4.4 that $\int_{0}^{\infty} L_{a}\left(B^{x, y}\right)^{2} \mathrm{~d} a$ is the limit in distribution of $N^{-1 / 3} \sum_{h \in N^{-1 / 3}\left(\mathbb{Z}_{>0}+1 / 2\right)} L_{h}\left(X^{x, y ; N, T_{N}}\right)^{2}, N \in \mathbb{N}$. The latter are stochastically dominated by $8 T\left(N^{-1 / 3} J_{T N^{2 / 3}}+N^{-1 / 3} \tilde{J}_{T N^{2 / 3}}+2|x-y|+2 N^{-1 / 3}\right), N \in \mathbb{N}$, respectively, where $J_{T N^{2 / 3}}, \tilde{J}_{T N^{2 / 3}}$ are the maxima of two independent simple random walks with $T N^{2 / 3}$ steps of size \pm 1 (see (4.19) and the paragraph following it). Passing to the limit $N \rightarrow \infty$, we conclude that $\int_{0}^{\infty} L_{a}\left(B^{x, y}\right)^{2} \mathrm{~d} a$ is stochastically dominated by $8 T(J+\tilde{J}+2|x-y|)$, where $J, \tilde{J}$ are the maxima of two independent standard Brownian motions on $[0, T]$. All in all, the expression in (5.2) is bounded above by

$$
\frac{C^{1 / 2}}{2 \pi T} \int_{\mathbb{R}_{\geq 0}} \int_{\mathbb{R}_{\geq 0}} e^{-\frac{(x-y)^{2}}{T}-\min (x, y)} \mathbb{E}\left[\exp \left(\frac{32 T}{\beta}(J+\tilde{J}+2|x-y|)\right)\right]^{1 / 2} \mathrm{~d} x \mathrm{~d} y .
$$

It remains to note the integrability of $e^{-\frac{(x-y)^{2}}{T}-\min (x, y)+\frac{32 T}{\beta}|x-y|}$ over $\left(\mathbb{R}_{\geq 0}\right)^{2}$.

Lemma 5.1 shows that the operators $\mathcal{U}(T)$ are well-defined, and we can now prove Propositions 2.2, 2.3 and 2.4. 
ProOf of Proposition 2.3. We need to show that the event

$$
\left\{\forall f \in L^{2}\left(\mathbb{R}_{\geq 0}\right): \mathcal{U}\left(T_{1}\right) \mathcal{U}\left(T_{2}\right) f=\mathcal{U}\left(T_{1}+T_{2}\right) f\right\}
$$

has probability one for any $T_{1}, T_{2} \geq 0$. Moreover, according to Lemma 5.1 , the operators $\mathcal{U}\left(T_{1}\right), \mathcal{U}\left(T_{2}\right), \mathcal{U}\left(T_{1}+T_{2}\right)$ are continuous with probability one, so that in the latter event we may replace $L^{2}\left(\mathbb{R}_{\geq 0}\right)$ by a countable dense subset. Consequently, it suffices to prove that $\mathcal{U}\left(T_{1}\right) \mathcal{U}\left(T_{2}\right) f=\mathcal{U}\left(T_{1}+T_{2}\right) f$ almost surely for a fixed $f \in L^{2}\left(\mathbb{R}_{\geq 0}\right)$. In addition, we may assume that $f \geq 0$, since otherwise we can decompose $f$ into its positive and negative parts. In this case, Fubini's theorem reveals that it is enough to show that almost surely

$$
\forall x, y \in \mathbb{R}_{\geq 0}: \quad \int_{\mathbb{R}_{\geq 0}} K\left(x, z ; T_{1}\right) K\left(z, y ; T_{2}\right) \mathrm{d} z=K\left(x, y ; T_{1}+T_{2}\right) .
$$

Further, elementary manipulations allow to rewrite the latter integral as

$$
\begin{aligned}
& \frac{1}{\sqrt{2 \pi\left(T_{1}+T_{2}\right)}} \int_{\mathbb{R}}\left(\frac{T_{1}+T_{2}}{2 \pi T_{1} T_{2}}\right)^{1 / 2} \\
& \cdot \exp \left(-\left(z-\frac{T_{1}}{T_{1}+T_{2}} y-\frac{T_{2}}{T_{1}+T_{2}} x\right)^{2} /\left(2 \frac{T_{1} T_{2}}{T_{1}+T_{2}}\right)\right) \\
& \cdot \mathbb{E}_{B^{x, z}, B^{z, y}}\left[\mathbf{1}_{\left\{\forall t: B^{x, z}(t) \geq 0, \forall t: B^{z, y}(t) \geq 0\right\}}\right. \\
& \cdot \exp \left(-\frac{(x-y)^{2}}{2\left(T_{1}+T_{2}\right)}-\frac{1}{2} \int_{0}^{T_{1}} B^{x, z}(t) \mathrm{d} t-\frac{1}{2} \int_{0}^{T_{2}} B^{z, y}(t) \mathrm{d} t\right. \\
& \left.\left.+\frac{1}{\sqrt{\beta}} \int_{0}^{\infty}\left(L_{a}\left(B^{x, z}\right)+L_{a}\left(B^{z, y}\right)\right) \mathrm{d} W(a)\right)\right] \mathrm{d} z .
\end{aligned}
$$

We now make the following observation: the process obtained by sampling a point $z$ according to the normal distribution with mean $\frac{T_{1}}{T_{1}+T_{2}} y+\frac{T_{2}}{T_{1}+T_{2}} x$ and variance $\frac{T_{1} T_{2}}{T_{1}+T_{2}}$ and then concatenating a standard Brownian bridge connecting $x$ to $z$ in time $T_{1}$ with a conditionally independent (given $z$ ) standard Brownian bridge connecting $z$ to $y$ in time $T_{2}$ is a standard Brownian bridge connecting $x$ to $y$ in time $T_{1}+T_{2}$. As a result, we end up with $K\left(x, y ; T_{1}+T_{2}\right)$.

Proof of Proposition 2.2. The almost sure symmetry of $\mathcal{U}(T)$ amounts to showing that the event

$$
\left\{\forall f, g \in L^{2}\left(\mathbb{R}_{\geq 0}\right): \int_{\mathbb{R}_{\geq 0}}(\mathcal{U}(T) f)(x) g(x) \mathrm{d} x=\int_{\mathbb{R}_{\geq 0}} f(x)(\mathcal{U}(T) g)(x) \mathrm{d} x\right\}
$$

has probability one. Thanks to the almost sure continuity of the operator $\mathcal{U}(T)$ (Lemma 5.1) it is further sufficient to consider $f, g$ from a countable dense subset 
of $L^{2}\left(\mathbb{R}_{\geq 0}\right)$. Hence, the symmetry property of $\mathcal{U}(T)$ reduces to the almost sure equality

$$
\int_{\mathbb{R}_{\geq 0}}(\mathcal{U}(T) f)(x) g(x) \mathrm{d} x=\int_{\mathbb{R}_{\geq 0}} f(x)(\mathcal{U}(T) g)(x) \mathrm{d} x
$$

for fixed $f, g \in L^{2}\left(\mathbb{R}_{\geq 0}\right)$. In addition, we may assume $f \geq 0, g \geq 0$, since otherwise we can decompose $f, g$ into their positive and negative parts. For such functions, we may use Fubini's theorem to reduce the statement further to: almost surely,

$$
\forall x, y \in \mathbb{R}_{\geq 0}: \quad K(x, y ; T)=K(y, x ; T) .
$$

The latter follows from the definition of the kernel $K(\cdot, \cdot ; T)$ and the fact that the time reversal of a standard Brownian bridge connecting $x$ to $y$ in time $T$ is a standard Brownian bridge connecting $y$ to $x$ in time $T$.

At this point, the almost sure nonnegativity of $\mathcal{U}(T)$ is a direct consequence of the almost sure identity $\mathcal{U}(T)=\mathcal{U}(T / 2) \mathcal{U}(T / 2)$ (Proposition 2.3) and the almost sure symmetry of $\mathcal{U}(T / 2)$.

So far, we have established that $\mathcal{U}(T)$ is a symmetric positive Hilbert-Schmidt operator with probability one. In particular, the spectral theorem for symmetric compact operators reveals that $L^{2}\left(\mathbb{R}_{\geq 0}\right)$ almost surely admits an orthonormal basis comprised of eigenfunctions of $\mathcal{U}(T)$ with the corresponding nonnegative eigenvalues $e^{1}(T) \geq e^{2}(T) \geq \cdots$. By definition, $\mathcal{U}(T)$ is trace class iff $\sum_{i=1}^{\infty} e^{i}(T)<\infty$, and in this case

$$
\operatorname{Trace}(\mathcal{U}(T))=\sum_{i=1}^{\infty} e^{i}(T)
$$

Moreover, $\mathcal{U}(T) \mathcal{U}(T / 2)=\mathcal{U}(T / 2) \mathcal{U}(T)$ and [47], Chapter 28, Theorem 7, show that $L^{2}\left(\mathbb{R}_{\geq 0}\right)$ almost surely admits an orthonormal basis comprised of common eigenfunctions of $\mathcal{U}(T)$ and $\mathcal{U}(T / 2)$. Consequently, $\sum_{i=1}^{\infty} e^{i}(T)$ can be rewritten as $\sum_{i=1}^{\infty} e^{i}(T / 2)^{2}$. The latter expression gives the square of the Hilbert-Schmidt norm of $\mathcal{U}(T / 2)$ (see [47], Chapter 30, Exercise 11) and can be therefore rewritten further as

$$
\int_{\mathbb{R}_{\geq 0}} \int_{\mathbb{R}_{\geq 0}} K(x, y ; T / 2)^{2} \mathrm{~d} x \mathrm{~d} y
$$

(see [58], Theorem 2.11). The latter expression is finite with probability one by Lemma 5.1, so that $\mathcal{U}(T)$ is trace class. Finally, an application of the semigroup equation (5.4) to

$$
\int_{\mathbb{R}_{\geq 0}} \int_{\mathbb{R}_{\geq 0}} K(x, y ; T / 2)^{2} \mathrm{~d} x \mathrm{~d} y=\int_{\mathbb{R}_{\geq 0}} \int_{\mathbb{R}_{\geq 0}} K(y, x ; T / 2) K(x, y ; T / 2) \mathrm{d} x \mathrm{~d} y
$$

gives the trace formula (2.5). 
PROOF OF PROPOSITION 2.4. We will show the stronger statement that, for each even integer $p \geq 2$, it holds

$$
\lim _{t \rightarrow T} \mathbb{E}\left[\|\mathcal{U}(T) f-\mathcal{U}(t) f\|^{p}\right]=0 .
$$

With the notation $\|\mathcal{U}(t)\|$ for the spectral norm (i.e., the largest eigenvalue) of $\mathcal{U}(t)$ we can use the Cauchy-Schwarz inequality to find

$$
\begin{aligned}
\mathbb{E} \| \mathcal{U} & (T) f-\mathcal{U}(t) f \|^{p} \\
& \leq \mathbb{E}\left[\|\mathcal{U}(T \wedge t)\|^{p}\|\mathcal{U}(|T-t|) f-f\|^{p}\right] \\
& \leq \mathbb{E}\left[\|\mathcal{U}(T \wedge t)\|^{2 p}\right]^{1 / 2} \mathbb{E}\left[\|\mathcal{U}(|T-t|) f-f\|^{2 p}\right]^{1 / 2} \\
& \leq \mathbb{E}[\operatorname{Trace}(\mathcal{U}(2 p(t \wedge T)))]^{1 / 2} \mathbb{E}\left[\|\mathcal{U}(|T-t|) f-f\|^{2 p}\right]^{1 / 2}
\end{aligned}
$$

The reduction of the trace to a Hilbert-Schmidt norm as in the proof of Proposition 2.2 and the arguments in the proof of Lemma 5.1 show that the quantity $\mathbb{E}[\operatorname{Trace}(\mathcal{U}(2 p \min (t, T)))]$ can be bounded uniformly for all $t$ in a neighborhood of a fixed $T>0$. Consequently, we only need to show (5.8) for $T=0$.

Write $\mathcal{G}(T)$ for the integral operator with kernel

$$
\begin{aligned}
K^{\mathcal{G}}(x, y ; T)= & \frac{1}{\sqrt{2 \pi T}} \exp \left(-\frac{(x-y)^{2}}{2 T}\right) \\
& \cdot \mathbb{E}_{B^{x, y}}\left[\mathbf{1}_{\left\{\forall t: B^{x, y}(t) \geq 0\right\}} \exp \left(-\frac{1}{2} \int_{0}^{T} B^{x, y}(t) \mathrm{d} t\right)\right] .
\end{aligned}
$$

As $T \rightarrow 0$, the operators $\mathcal{G}(T)$ converge strongly to $\mathcal{U}(0)$ (= the identity operator); see, for example, [49], Section 2, Step 6. Therefore, it is enough to prove

$$
\lim _{T \rightarrow 0} \mathbb{E}\left[\|\mathcal{U}(T) f-\mathcal{G}(T) f\|^{p}\right]=0
$$

or, in other words, the convergence to 0 as $T \rightarrow 0$ of

$$
\begin{aligned}
\mathbb{E}\left[\left(\int _ { \mathbb { R } _ { \geq 0 } } \left(\int_{\mathbb{R}_{\geq 0}} \frac{1}{\sqrt{2 \pi T}} e^{-\frac{(x-y)^{2}}{2 T}}\right.\right.\right. \\
\cdot \mathbb{E}_{B^{x, y}}\left[\mathbf{1}_{\left\{\forall t: B^{x, y}(t) \geq 0\right\}} \exp \left(-\frac{1}{2} \int_{0}^{T} B^{x, y}(t) \mathrm{d} t\right)\right. \\
\left.\left.\left.\left.\cdot\left(\exp \left(\frac{1}{\sqrt{\beta}} \int_{0}^{\infty} L_{a}\left(B^{x, y}\right) \mathrm{d} W(a)\right)-1\right)\right] f(y) \mathrm{d} y\right)^{2} \mathrm{~d} x\right)^{p / 2}\right] .
\end{aligned}
$$

Next, we apply the elementary inequality $\left|e^{S}-1\right| \leq|S| e^{|S|}, S \in \mathbb{R}$ with

$$
S:=\frac{1}{\sqrt{\beta}} \int_{0}^{\infty} L_{a}\left(B^{x, y}\right) \mathrm{d} W(a)
$$


to estimate the expression in (5.10) by

$$
\begin{aligned}
\mathbb{E}\left[\left(\int _ { \mathbb { R } _ { \geq 0 } } \left(\int_{\mathbb{R}_{\geq 0}} \frac{1}{\sqrt{2 \pi T}} \exp \left(-\frac{(x-y)^{2}}{2 T}\right)\right.\right.\right. \\
\quad \cdot \mathbb{E}_{B^{x, y}}\left[\mathbf{1}_{\left\{\forall t: B^{x, y}(t) \geq 0\right\}}\right. \\
\left.\left.\left.\left.\quad \cdot \exp \left(-\frac{1}{2} \int_{0}^{T} B^{x, y}(t) \mathrm{d} t\right)|S| e^{|S|}\right]|f(y)| \mathrm{d} y\right)^{2} \mathrm{~d} x\right)^{p / 2}\right] .
\end{aligned}
$$

Moreover, writing the square of the inner integral and then the $p / 2$ power of the outer integral as products of integrals in independent variables and applying $\mathrm{Fu}-$ bini's theorem together with Hölder's inequality we end up with the bound

$$
\begin{aligned}
\left(\int_{\mathbb{R}_{\geq 0}}\right. & \left(\int_{\mathbb{R}_{\geq 0}} \frac{1}{\sqrt{2 \pi T}} \exp \left(-\frac{(x-y)^{2}}{2 T}\right)\right. \\
\cdot & \mathbb{E}_{B^{x, y}}\left[\mathbf{1}_{\left\{\forall t: B^{x, y}(t) \geq 0\right\}} \exp \left(-\frac{1}{2} \int_{0}^{T} B^{x, y}(t) \mathrm{d} t\right)\right. \\
\cdot & \left.\left.\left.\mathbb{E}_{W}\left[|S|^{p} e^{p|S|}\right]^{1 / p}\right]|f(y)| \mathrm{d} y\right)^{2} \mathrm{~d} x\right)^{p / 2} .
\end{aligned}
$$

Dropping the indicator function and using the Cauchy-Schwarz inequality we obtain the further estimate

$$
\begin{aligned}
\left(\int_{\mathbb{R}_{\geq 0}}\right. & \left(\int_{\mathbb{R}_{\geq 0}} \frac{1}{\sqrt{2 \pi T}} \exp \left(-\frac{(x-y)^{2}}{2 T}\right)|f(y)|\right. \\
\cdot & \mathbb{E}_{B^{x, y}}\left[\exp \left(-\int_{0}^{T} B^{x, y}(t) \mathrm{d} t\right)\right]^{1 / 2} \\
\cdot & \left.\left.\mathbb{E}_{B^{x, y}}\left[\mathbb{E}_{W}\left[|S|^{p} e^{p|S|}\right]^{2 / p}\right]^{1 / 2} \mathrm{~d} y\right)^{2} \mathrm{~d} x\right)^{p / 2} .
\end{aligned}
$$

Now, the same argument as in the proof of Lemma 5.1 shows that the first expectation with respect to $B^{x, y}$ is bounded by a uniform constant for all $x, y \in$ $\mathbb{R}_{\geq 0}$ and $T \in(0,1]$. To control the second expectation with respect to $B^{x, y}$, we combine the inequality $|S|^{p} \leq p ! e^{|S|}$, the representation

$$
B^{x, y}(t)=\sqrt{T} \hat{B}^{x / \sqrt{T}, y / \sqrt{T}}(t / T), \quad t \in[0, T]
$$

with a standard Brownian bridge $\hat{B}^{x / \sqrt{T}, y / \sqrt{T}}$ connecting $x / \sqrt{T}$ to $y / \sqrt{T}$ in time 1 , the fact that for a given trajectory of $\hat{B}^{x / \sqrt{T}, y / \sqrt{T}}$ the random variable $S$ has the normal distribution with mean 0 and variance

$$
\frac{T^{3 / 2}}{\beta} \int_{0}^{\infty} L_{a}\left(\hat{B}^{x / \sqrt{T}, y / \sqrt{T}}\right)^{2} \mathrm{~d} a,
$$


and a bound on the integral of the squared local times as in the proof of Lemma 5.1 to obtain an estimate of the form

$$
\mathbb{E}_{B^{x, y}}\left[\mathbb{E}_{W}\left[|S|^{p} e^{p|S|}\right]^{2 / p}\right]^{1 / 2} \leq C T^{3 / 2} e^{C|x-y| / \sqrt{T}} .
$$

Here, $C<\infty$ is a constant depending only on $p$.

All in all, we end up with the following bound on the expression in (5.10):

$$
C T^{p}\left(\int_{\mathbb{R}_{\geq 0}}\left(\int_{\mathbb{R}_{\geq 0}} \exp \left(-\frac{(x-y)^{2}}{2 T}+C \frac{|x-y|}{\sqrt{T}}\right)|f(y)| \mathrm{d} y\right)^{2} \mathrm{~d} x\right)^{p / 2} .
$$

By Young's inequality for convolution the latter is at most

$$
C T^{p}\|f\|^{p}\left(\int_{\mathbb{R}} \exp \left(-\frac{x^{2}}{2 T}+C \frac{|x|}{\sqrt{T}}\right) \mathrm{d} x\right)^{p} \leq C T^{3 p / 2}\|f\|^{p},
$$

which completes the proof.

6. Convergence of extreme eigenvalues. The aim of this section is to obtain Corollary 2.1 from Theorem 2.1. Such proofs are standard in the literature on the moments method for random matrices; see, for example, [61], [60], Section 5, [59]. Nevertheless, we give a sketch of the proof for the sake of completeness. In addition, it yields Proposition 2.5. We start with the following statement.

\section{LEMMA 6.1. The convergence in distribution}

$$
\sum_{i=1}^{N} e^{T \lambda_{N}^{i} / 2} \longrightarrow_{N \rightarrow \infty} \operatorname{Trace}(\mathcal{U}(T))
$$

holds jointly for any finitely many $T$ 's.

PROOF. We write $\mu_{N}^{1,+} \geq \mu_{N}^{2,+} \geq \cdots$ and $\mu_{N}^{1,-} \leq \mu_{N}^{2,-} \leq \cdots$ for the positive and the negative eigenvalues of $\bar{M}_{N}$, respectively. In addition, we define their rescaled versions

$$
\begin{aligned}
& \lambda_{N}^{i,+}=N^{1 / 6}\left(\mu_{N}^{i,+}-2 \sqrt{N}\right) \quad \text { and } \\
& \lambda_{N}^{i,-}=-N^{1 / 6}\left(\mu_{N}^{i,-}+2 \sqrt{N}\right), \quad i=1,2, \ldots
\end{aligned}
$$

Clearly,

$\operatorname{Trace}(\mathcal{M}(T, N))$

$$
\begin{aligned}
= & \frac{1}{2} \sum_{i}\left(1+\frac{\lambda_{N}^{i,+}}{2 N^{2 / 3}}\right)^{\left\lfloor T N^{2 / 3}\right\rfloor}+\frac{(-1)^{\left\lfloor T N^{2 / 3}\right\rfloor}}{2} \sum_{i}\left(1+\frac{\lambda_{N}^{i,-}}{2 N^{2 / 3}}\right)^{\left\lfloor T N^{2 / 3}\right\rfloor} \\
& +\frac{1}{2} \sum_{i}\left(1+\frac{\lambda_{N}^{i,+}}{2 N^{2 / 3}}\right)^{\left\lfloor T N^{2 / 3}\right\rfloor-1} \\
& +\frac{(-1)^{\left\lfloor T N^{2 / 3}\right\rfloor-1}}{2} \sum_{i}\left(1+\frac{\lambda_{N}^{i,-}}{2 N^{2 / 3}}\right)^{\left\lfloor T N^{2 / 3}\right\rfloor-1} .
\end{aligned}
$$


From Theorem 2.1, we know that both sides of (6.2) converge in distribution to the right-hand side of (6.1) in the limit $N \rightarrow \infty$. Consequently, it suffices to show the convergence to 0 in probability as $N \rightarrow \infty$ of the difference between the righthand side of (6.2) and

$$
\begin{aligned}
\sum_{i=1}^{N} e^{T \lambda_{N}^{i} / 2}= & \frac{1}{2} \sum_{i=1}^{N} e^{T \lambda_{N}^{i} / 2}+\frac{1}{2} \sum_{i=1}^{N} e^{T \lambda_{N}^{i} / 2}+\frac{(-1)^{\left\lfloor T N^{2 / 3}\right\rfloor}}{2} \sum_{i=1}^{N} e^{T \lambda_{N}^{i} / 2} \\
& +\frac{(-1)^{\left\lfloor T N^{2 / 3}\right\rfloor-1}}{2} \sum_{i=1}^{N} e^{T \lambda_{N}^{i} / 2}
\end{aligned}
$$

To analyze the difference between the right-hand sides of (6.2) and (6.3), we take $\varepsilon=1 / 100$ and distinguish between four types of (rescaled) eigenvalues:

1. "bulk eigenvalues": $\lambda_{N}^{i,+}$ 's and $\lambda_{N}^{i,-}$ 's which are less or equal to $-N^{\varepsilon}$,

2. "outliers": $\lambda_{N}^{i,+}$ 's and $\lambda_{N}^{i,-}$ 's which are greater or equal to $N^{\varepsilon}$,

3. "right edge eigenvalues": $\lambda_{N}^{i,+}$, s in $\left(-N^{\varepsilon}, N^{\varepsilon}\right)$,

4. "left edge eigenvalues": $\lambda_{N}^{i,-}$ 's in $\left(-N^{\varepsilon}, N^{\varepsilon}\right)$.

The contribution of the bulk eigenvalues to the right-hand side of (6.3) becomes negligible in the limit $N \rightarrow \infty$, since there are at most $N$ of them and each contributes at most $e^{-T N^{\varepsilon} / 2}$. Due to the elementary inequality $1+a \leq e^{a}, a \in \mathbb{R}$, the same is true for the right-hand side of (6.2).

Next, we show that with probability tending to 1 as $N \rightarrow \infty$ there are no outliers. To this end, we consider a sequence $T_{N}, N \in \mathbb{N}$ of positive numbers such that $T_{N} N^{2 / 3}, N \in \mathbb{N}$ are even integers and $\sup _{N}\left|T_{N}-T\right| N^{2 / 3}<\infty$. Then

$$
\operatorname{Trace}\left(\left(M_{N}\right)^{T_{N} N^{2 / 3}}\right)=\sum_{i}\left(1+\frac{\lambda_{N}^{i,+}}{2 N^{2 / 3}}\right)^{T_{N} N^{2 / 3}}+\sum_{i}\left(1+\frac{\lambda_{N}^{i,-}}{2 N^{2 / 3}}\right)^{T_{N} N^{2 / 3}} .
$$

Hence, if there is an outlier, then

$$
\operatorname{Trace}\left(\left(M_{N}\right)^{T_{N} N^{2 / 3}}\right) \geq\left(1+\frac{N^{\varepsilon}}{2 N^{2 / 3}}\right)^{T_{N} N^{2 / 3}} .
$$

According to Remark 4.5, the left-hand side of (6.4) converges in distribution as $N \rightarrow \infty$. On the other hand, the right-hand side of (6.4) becomes arbitrarily large in the same limit. Consequently, the probability that the inequality (6.4) takes place tends to 0 as $N \rightarrow \infty$.

Finally, to the summands involving the edge eigenvalues we can apply the approximations

$$
\left(1+\frac{\lambda_{N}^{i, \pm}}{2 N^{2 / 3}}\right)^{\left\lfloor T N^{2 / 3}\right\rfloor} \approx e^{T \lambda_{N}^{i, \pm} / 2}, \quad\left(1+\frac{\lambda_{N}^{i, \pm}}{2 N^{2 / 3}}\right)^{\left\lfloor T N^{2 / 3}\right\rfloor-1} \approx e^{T \lambda_{N}^{i, \pm} / 2}
$$


with additive errors of at most

$$
\begin{aligned}
& \left(e^{N^{-2 / 3+2 \varepsilon} / 2}-1\right)\left(1+\frac{\lambda_{N}^{i, \pm}}{2 N^{2 / 3}}\right)^{\left\lfloor T N^{2 / 3}\right\rfloor}, \\
& \left(e^{N^{-2 / 3+2 \varepsilon} / 2}-1\right)\left(1+\frac{\lambda_{N}^{i, \pm}}{2 N^{2 / 3}}\right)^{\left\lfloor T N^{2 / 3}\right\rfloor-1},
\end{aligned}
$$

respectively. It remains to show that the sums of the latter errors over all the edge eigenvalues tend to 0 in probability. To this end, it suffices to prove that the expressions

$$
\left(e^{N^{-2 / 3+2 \varepsilon} / 2}-1\right) \sum_{i=1}^{N}\left(1+\frac{\lambda_{N}^{i}}{2 N^{2 / 3}}\right)^{\left\lfloor T N^{2 / 3}\right\rfloor-\epsilon}, \quad \epsilon \in\{0,1\}
$$

converge to 0 in probability, since the contributions of the eigenvalues away from edge to them have been shown to be negligible before. The latter convergences are direct consequences of Remark 4.5.

We can now deduce Corollary 2.1 and Proposition 2.5. According to Proposition 2.2, for each $T>0, \mathcal{U}(T)$ is a symmetric trace class operator with probability one. In particular, $\mathcal{U}(T)$ is compact (see, e.g., [47], Section 30.8, Exercise $11(\mathrm{~h}),(\mathrm{g}))$ and, therefore, its spectrum is discrete. Moreover, the almost sure commutativity of operators $\mathcal{U}(T), T>0$ shows that there exists an orthonormal basis $\mathbf{v}^{1}, \mathbf{v}^{2}, \ldots$ of $L^{2}\left(\mathbb{R}_{\geq 0}\right)$ consisting of eigenfunctions common to all $\mathcal{U}(T)$, $T \in(0, \infty) \cap \mathbb{Q}$ (see, e.g., [47], Chapter 28, Theorem 7). Next, we order the eigenvectors in such a way that the corresponding eigenvalues of $\mathcal{U}(1)$ satisfy $e^{1} \geq e^{2} \geq \cdots$, note that all of the latter are nonnegative $[\mathcal{U}(1)$ is a nonnegative operator], and define $\eta^{1} \geq \eta^{2} \geq \cdots$ by $\eta^{i}=2 \log e^{i}, i \in \mathbb{N}$.

The semigroup property shows further that $\mathcal{U}(T) \mathbf{v}^{i}=\exp \left(T \eta^{i} / 2\right) \mathbf{v}^{i}, i \in \mathbb{N}$ for all $T \in(0, \infty) \cap \mathbb{Q}$ and, in particular, $\operatorname{Trace}(\mathcal{U}(T))=\sum_{i=1}^{\infty} \exp \left(T \eta^{i} / 2\right)$. We remark that a priori Proposition 2.2 allows some of the eigenvalues $e^{i}$ of $\mathcal{U}(1)$ to vanish. However, if that were the case, all of the operators $\mathcal{U}(T), T \in(0, \infty) \cap \mathbb{Q}$ would evaluate to 0 on the corresponding eigenvectors, contradicting the continuity of the semigroup at $T=0$ established in Proposition 2.4.

At this point, Lemma 6.1 implies the convergences

$$
\sum_{i=1}^{N} e^{T \lambda_{N}^{i} / 2} \longrightarrow N \rightarrow \infty \sum_{i=1}^{\infty} e^{T \eta^{i} / 2}
$$

in distribution, jointly for any finitely many $T$ 's in $(0, \infty) \cap \mathbb{Q}$. Applying the Skorokhod representation theorem in the form of [24], Theorem 3.5.1, we find a new probability space, on which the convergence of (6.5) holds almost surely for all $T \in(0, \infty) \cap \mathbb{Q}$. Then the one-to-one property of Laplace transforms implies that, for all $i \in \mathbb{N}$, we must have the almost sure convergence $\lim _{N \rightarrow \infty} \lambda^{i}=\eta^{i}$; 
see, for example, [60], Section 5, for more details. This proves (2.10). Finally, Proposition 2.5 for irrational $T>0$ follows from the continuity of the semigroup (Proposition 2.5), which then implies $\operatorname{Trace}(\mathcal{U}(T))=\sum_{i=1}^{\infty} \exp \left(T \eta^{i} / 2\right)$ and (6.5) via Lemma 6.1 as before.

7. Properties of the stochastic Airy semigroup II. PROOF OF COROLLARY 2.2. Note that the matrix $M_{N}$ can be interpreted as an operator on $L^{2}\left(\mathbb{R}_{\geq 0}\right)$ : for a function $f \in L^{2}\left(\mathbb{R}_{\geq 0}\right)$, one can define the action of $M_{N}$ on $f$ by interpreting the $N$-dimensional vector $M_{N}\left(\pi_{N} f\right)$ as the piecewise constant function on $\left[0, N^{-1 / 3}\right), \ldots,\left[N^{2 / 3}-N^{-1 / 3}, N^{2 / 3}\right)$ whose values are given by the $N^{1 / 6}$ multiples of the entries of $M_{N}\left(\pi_{N} f\right)$. The main result of [56] (see also [44]) provides a coupling of the matrices $M_{N}, N \in \mathbb{N}$ [viewed as operators on $L^{2}\left(\mathbb{R}_{\geq 0}\right)$ ] such that their scaled largest eigenvalues and the corresponding eigenfunctions converge to those of $-\frac{1}{2} S A O_{\beta}$ almost surely. Simultaneously, the Brownian motion $W$ arises via the limit transition as in (2.7).

It is not hard to see that under the coupling of [56] the largest eigenvalues and the corresponding eigenfunctions of the matrices $\mathcal{M}\left(T, \mathbb{R}_{\geq 0}, N\right), N \in \mathbb{N}$ [viewed as operators on $\left.L^{2}\left(\mathbb{R}_{\geq 0}\right)\right]$ converge to those of $e^{-\frac{T}{2} S A O_{\beta}}$ almost surely. Indeed, for the convergence of eigenfunctions it suffices to observe that the eigenvectors of each $\mathcal{M}\left(T, \mathbb{R}_{\geq 0}, N\right)$ are the same as those of the corresponding matrix $M_{N}$, and the eigenfunctions of $e^{-\frac{T}{2} S A O_{\beta}}$ are the same as those of $-\frac{1}{2} S A O_{\beta}$. On the other hand, for the convergence of eigenvalues we can use the same approximation of the normalized high powers of eigenvalues by the exponentials of their scaled versions as in the proof of Lemma 6.1.

Moreover, the almost sure convergence of the eigenvalues of $-\frac{1}{2} S A O_{\beta}$ to $-\infty$ ([56], Proposition 3.5) implies the almost sure strong convergence of the matrices $\mathcal{M}\left(T, \mathbb{R}_{\geq 0}, N\right), N \in \mathbb{N}$ [viewed as operators on $L^{2}\left(\mathbb{R}_{\geq 0}\right)$ ] to $e^{-\frac{T}{2} S A O_{\beta}}$. Since strong convergence implies weak convergence, by comparing with Theorem 2.1 we conclude that the finite-dimensional distributions of the families $\int_{\mathbb{R}_{\geq 0}} f(x)\left(e^{-\frac{T}{2} S A O_{\beta}} g\right)(x) \mathrm{d} x, f, g \in L^{2}\left(\mathbb{R}_{\geq 0}\right)$ and $\int_{\mathbb{R}_{\geq 0}} f(x)(\mathcal{U}(T) g)(x) \mathrm{d} x, f, g \in$ $L^{2}\left(\mathbb{R}_{\geq 0}\right)$ coincide, as well as their joint distributions with the Brownian motions $W$ in the definitions of $S A O_{\beta}$ and $\mathcal{U}(T)$, respectively.

In other words, the laws of the pairs $\left(W, e^{-\frac{T}{2} S A O_{\beta}}\right)$ and $(W, \mathcal{U}(T))$ are the same, where the second component is endowed with the Borel $\sigma$-algebra associated with the weak operator topology. Putting together the measurability of both $e^{-\frac{T}{2} S A O_{\beta}}$ and $\mathcal{U}(T)$ with respect to the $\sigma$-algebra generated by $W$ with [40], Theorem 5.3, we conclude that there is a unique up to null sets of $W$ deterministic functional $F$ such that $(W, F(W))$ has the same law as each of the pairs $\left(W, e^{-\frac{T}{2} S A O_{\beta}}\right)$ and $(W, \mathcal{U}(T))$. Consequently, if we choose the Brownian motions $W$ in the respective definitions of $S A O_{\beta}$ and $\mathcal{U}(T)$ to be the same, the almost sure identities $e^{-\frac{T}{2} S A O_{\beta}}=$ $F(W)=\mathcal{U}(T)$ will hold. 
We conclude the section with the proof of Proposition 2.7 and Corollary 2.3.

Proof of Proposition 2.7 And Corollary 2.3. Fix a $T>0$. From Corollary 2.2, we know that the expectation $\mathbb{E}\left[\sum_{i=1}^{\infty} e^{T \eta^{i} / 2}\right]$ is given by $\mathbb{E}[\operatorname{Trace}(\mathcal{U}(T))]$ which, in turn, can be computed to $\mathbb{E}\left[\int_{\mathbb{R}_{>0}} K(x, x ; T) \mathrm{d} x\right]$ thanks to the trace formula (2.5). To simplify the latter expectation, we apply Fubini's theorem and take the expectation with respect to $W$ first using Novikov's condition (see, e.g., [42], Proposition 3.5.12) to obtain

$$
\begin{aligned}
& \frac{1}{\sqrt{2 \pi T}} \int_{\mathbb{R}_{\geq 0}} \mathbb{E}_{B^{x, x}}\left[\mathbf{1}_{\left\{\forall t: B^{x, x}(t) \geq 0\right\}}\right. \\
& \left.\quad \cdot \exp \left(-\frac{1}{2} \int_{0}^{T} B^{x, x}(t) \mathrm{d} t+\frac{1}{2 \beta} \int_{0}^{\infty} L_{a}\left(B^{x, x}\right)^{2} \mathrm{~d} a\right)\right] \mathrm{d} x .
\end{aligned}
$$

Next, we note that $B^{0,0}:=B^{x, x}-x$ is a standard Brownian bridge connecting 0 to 0 in time $T$, in terms of which the latter expression reads

$$
\begin{aligned}
& \frac{1}{\sqrt{2 \pi T}} \int_{\mathbb{R}_{\geq 0}} \mathbb{E}_{B^{0,0}}\left[\mathbf{1}_{\left\{\forall t: x \geq-B^{0,0}(t)\right\}}\right. \\
& \left.\cdot \exp \left(-\frac{1}{2} \int_{0}^{T}\left(B^{0,0}(t)+x\right) \mathrm{d} t+\frac{1}{2 \beta} \int_{-\infty}^{\infty} L_{a}\left(B^{0,0}\right)^{2} \mathrm{~d} a\right)\right] \mathrm{d} x .
\end{aligned}
$$

At this point, we rewrite the event $\left\{\forall t: x \geq-B^{0,0}(t)\right\}$ as $\left\{x \geq-\min \left(B^{0,0}\right)\right\}$ and compute the integral with respect to $x$ before taking the expectation

$$
\begin{aligned}
& \sqrt{\frac{2}{\pi}} T^{-3 / 2} \mathbb{E}_{B^{0,0}}\left[\operatorname { e x p } \left(-\frac{1}{2} \int_{0}^{T}\left(B^{0,0}(t)-\min \left(B^{0,0}\right)\right) \mathrm{d} t\right.\right. \\
& \left.\left.+\frac{1}{2 \beta} \int_{-\infty}^{\infty} L_{a}\left(B^{0,0}\right)^{2} \mathrm{~d} a\right)\right] .
\end{aligned}
$$

Lastly, consider the Vervaat transform $V\left(B^{0,0}\right)(t):=B^{0,0}\left(\left(t+t^{*}\right) \bmod T\right)-$ $\min \left(B^{0,0}\right), t \in[0, T]$, where $t^{*}$ is the almost surely unique time at which $B^{0,0}$ attains its global minimum $\min \left(B^{0,0}\right)$. By Vervaat's theorem ([66], Theorem 1$), V\left(B^{0,0}\right)$ is a standard Brownian excursion on $[0, T]$, so that $\mathfrak{e}(t):=$ $T^{-1 / 2} V\left(B^{0,0}\right)(T t), t \in[0,1]$ is a standard Brownian excursion on $[0,1]$. The quantity of (7.1) can be now reexpressed in terms of $\mathfrak{e}$ as

$$
\sqrt{\frac{2}{\pi}} T^{-3 / 2} \mathbb{E}\left[\exp \left(-\frac{T^{3 / 2}}{2} \int_{0}^{1} \mathfrak{e}(t) \mathrm{d} t+\frac{T^{3 / 2}}{2 \beta} \int_{0}^{\infty}\left(l_{y}\right)^{2} \mathrm{~d} y\right)\right],
$$

where each $l_{y}$ is the total local time of $\mathfrak{e}$ at level $y$. Proposition 2.7 readily follows.

In addition, for $\beta=2$, we can equate the results of Propositions 2.7 and 2.6 to obtain

$$
\mathbb{E}\left[\exp \left(-\frac{T^{3 / 2}}{2}\left(\int_{0}^{1} \mathfrak{e}(t) \mathrm{d} t-\frac{1}{2} \int_{0}^{\infty}\left(l_{y}\right)^{2} \mathrm{~d} y\right)\right)\right]=e^{T^{3} / 96}
$$


Since $T>0$ was arbitrary, we have identified the moment generating function on the negative half-line of the random variable $\int_{0}^{1} \mathfrak{e}(t) \mathrm{d} t-\frac{1}{2} \int_{0}^{\infty}\left(l_{y}\right)^{2} \mathrm{~d} y$ with that of a Gaussian random variable of mean 0 and variance $\frac{1}{12}$. The usual analytic continuation technique via Morera's theorem allows to extend such identity to the open left complex half-plane. Corollary 2.3 now follows from the uniqueness theorem for moment generating functions (see, e.g., [67], Chapter VI, Theorem 6a).

Acknowledgments. We would like to thank Simone Warzel for suggesting the strategy for the proof of the trace formula (2.5), and Sasha Sodin for helpful discussions. We thank Pierre Yves Gaudreau Lamarre for pointing out several inaccuracies in the first version of the text.

\section{SUPPLEMENTARY MATERIAL}

Global asymptotics (DOI: 10.1214/17-AOP1229SUPPA; .pdf). We analyze the asymptotics of global linear statistics of the spectrum of symmetric tridiagonal matrices and prove a cental limit theorem for them.

Coupling with Brownian bridge local times (DOI: 10.1214/17-AOP1229 SUPPB; .pdf). We construct a coupling with the local times of the Brownian bridge and prove Proposition 4.1.

\section{REFERENCES}

[1] Akemann, G., Baik, J. and Di Francesco, P., eds. (2011). The Oxford Handbook of Random Matrix Theory. Oxford Univ. Press, Oxford. MR2920518

[2] Anderson, G. W., Guionnet, A. and Zeitouni, O. (2010). An Introduction to Random Matrices. Cambridge Studies in Advanced Mathematics 118. Cambridge Univ. Press, Cambridge. MR2760897

[3] Assaf, S., Forman, N. and Pitman, J. (2015). The quantile transform of simple walks and Brownian motion. Electron. J. Probab. 20 no. 90, 39. MR3399826

[4] Bass, R. F. and Khoshnevisan, D. (1995). Laws of the iterated logarithm for local times of the empirical process. Ann. Probab. 23 388-399. MR1330775

[5] Bekerman, F., Figalli, A. and Guionnet, A. (2015). Transport maps for $\beta$-matrix models and universality. Comm. Math. Phys. 338 589-619. MR3351052

[6] Bhattacharya, R. N. and Ranga RaO, R. (1976). Normal Approximation and Asymptotic Expansions. Wiley, New York. MR0436272

[7] Biane, PH. and Yor, M. (1987). Valeurs principales associées aux temps locaux browniens. Bull. Sci. Math. (2) 111 23-101. MR0886959

[8] Billingsley, P. (1968). Convergence of Probability Measures, 2nd ed. Wiley, New York. MR0233396

[9] Bloemendal, A. (2011). Finite rank perturbations of random matrices and their continuum limits. Ph.D. thesis, Univ. Toronto. Available at www.collectionscanada.gc.ca/ obj/thesescanada/vol2/OTU/TC-OTU-31693.pdf. MR3004406

[10] Borodin, A. and Corwin, I. (2014). Macdonald processes. Probab. Theory Related Fields 158 225-400. MR3152785

[11] Borodin, A., Corwin, I., Gorin, V. and Shakirov, S. (2016). Observables of Macdonald processes. Trans. Amer. Math. Soc. 368 1517-1558. 
[12] Borodin, A. and Gorin, V. (2015). General $\beta$-Jacobi corners process and the Gaussian free field. Comm. Pure Appl. Math. 68 1774-1844. MR3385342

[13] Borodin, A. and Gorin, V. (2015). Contour integral formulas for Jacobi ensemble. In appendix to "Moments of the position of the maximum for GUE characteristic polynomials and for log-correlated Gaussian processes" by Y. V. Fyodorov, P. Le Doussal. Preprint. Available at arXiv:1511.04258.

[14] Borodin, A. and Gorin, V. (2016). Lectures on integrable probability. In Probability and Statistical Physics in St. Petersburg. Proc. Sympos. Pure Math. 91 155-214. Amer. Math. Soc., Providence, RI. MR3526828

[15] Borodin, A. and Petrov, L. (2014). Integrable probability: From representation theory to Macdonald processes. Probab. Surv. 11 1-58. MR3178541

[16] Borodin, A. N. (1986). On the character of convergence to Brownian local time. II. Probab. Theory Related Fields 72 251-277. MR0836277

[17] Bourgade, P., Erdös, L. and YaU, H.-T. (2014). Edge universality of beta ensembles. Comm. Math. Phys. 332 261-353. MR3253704

[18] Corwin, I. (2014). Macdonald processes, quantum integrable systems and the Kardar-ParisiZhang universality class. In Proceedings of the ICM. Available at arXiv:1403.6877.

[19] CoRwin, I. and HAMmond, A. (2014). Brownian Gibbs property for Airy line ensembles. Invent. Math. 195 441-508. MR3152753

[20] CsÖrgő, M. and RÉVÉSZ, P. (1981). Strong Approximations in Probability and Statistics. Academic Press, New York. MR0666546

[21] CsÖRGŐ, M., SHI, Z. and Yor, M. (1999). Some asymptotic properties of the local time of the uniform empirical process. Bernoulli 5 1035-1058. MR1735784

[22] Deift, P. and Gioev, D. (2007). Universality at the edge of the spectrum for unitary, orthogonal, and symplectic ensembles of random matrices. Comm. Pure Appl. Math. 60 867-910. MR2306224

[23] Dembo, A. and Zeitouni, O. (1998). Large Deviations Techniques and Applications, 2nd ed. Applications of Mathematics (New York) 38. Springer, New York. MR1619036

[24] Dudley, R. M. (1999). Uniform Central Limit Theorems. Cambridge Studies in Advanced Mathematics 63. Cambridge Univ. Press, Cambridge. MR1720712

[25] Dumitriu, I. (2003). Eigenvalue statistics for beta-ensembles. Ph.D. thesis, Massachusetts Institute of Technology. Available at www.math.washington.edu/ dumitriu/main.pdf.

[26] Dumitriu, I. and Edelman, A. (2002). Matrix models for beta ensembles. J. Math. Phys. 43 5830-5847. MR1936554

[27] Dumitriu, I. and Edelman, A. (2006). Global spectrum fluctuations for the $\beta$-Hermite and $\beta$-Laguerre ensembles via matrix models. J. Math. Phys. 47 063302, 36. MR2239975

[28] DuY, T. K. (2016). On spectral measures of random Jacobi matrices. Preprint. Available at arXiv:1601.01146.

[29] Edelman, A. (2013). Stochastic Differential Equations and Random Matrices. Talk at the SIAM-03 Applied Linear Algebra Meeting in Williamsburg, VA.

[30] Edelman, A. and Sutton, B. D. (2007). From random matrices to stochastic operators. J. Stat. Phys. 127 1121-1165. MR2331033

[31] FelleR, W. (1967). An Introduction to Probability Theory and Its Applications. Vol. I, 3rd ed. Wiley, New York. MR0038583

[32] Forrester, P. J. (2010). Log-Gases and Random Matrices. London Mathematical Society Monographs Series 34. Princeton Univ. Press, Princeton, NJ. MR2641363

[33] Ghosh, S. and Peres, Y. (2017). Rigidity and tolerance in point processes: Gaussian zeros and Ginibre eigenvalues. Duke Math. J. 166 1789-1858. MR3679882

[34] Gorin, V. and ShKolnikov, M. (2018). Supplement to "Stochastic Airy semigroup through tridiagonal matrices." DOI:10.1214/17-AOP1229SUPPA, DOI:10.1214/17AOP1229SUPPB. 
[35] HARIYA, Y. (2016). A pathwise interpretation of the Gorin-Shkolnikov identity. Electron. Commun. Probab. 21 Paper No. 52, 6. MR3533284

[36] JANSON, S. (2007). Brownian excursion area, Wright's constants in graph enumeration, and other Brownian areas. Probab. Surv. 4 80-145. MR2318402

[37] Jeulin, T. (1985). Application de la theorie du grossissement a l'etude des temps locaux browniens. In Grossissements de Filtrations: Exemples et Applications. Lecture Notes in Math. 1118 197-304.

[38] JoHANSSON, K. (1998). On fluctuations of eigenvalues of random Hermitian matrices. Duke Math. J. 91 151-204. MR1487983

[39] Johansson, K. (2006). Random matrices and determinantal processes. In Mathematical Statistical Physics 1-55. Elsevier, Amsterdam. MR2581882

[40] Kallenberg, O. (2002). Foundations of Modern Probability, 2nd ed. Springer, New York. MR1876169

[41] Karandikar, R. L. (1995). On pathwise stochastic integration. Stochastic Process. Appl. 57 11-18. MR1327950

[42] Karatzas, I. and Shreve, S. E. (1991). Brownian Motion and Stochastic Calculus, 2nd ed. Graduate Texts in Mathematics 113. Springer, New York. MR1121940

[43] Khoshnevisan, D. (1992). Level crossings of the empirical process. Stochastic Process. Appl. 43 331-343. MR1191155

[44] Krishnapur, M., Rider, B. and VirÁG, B. (2016). Universality of the stochastic Airy operator. Comm. Pure Appl. Math. 69 145-199. MR3433632

[45] Laurent, B. and Massart, P. (2000). Adaptive estimation of a quadratic functional by model selection. Ann. Statist. 28 1302-1338. MR1805785

[46] Lawler, G. F. and Trujillo Ferreras, J. A. (2007). Random walk loop soup. Trans. Amer. Math. Soc. 359 767-787. MR2255196

[47] LAX, P. D. (2002). Functional Analysis. Wiley, New York. MR1892228

[48] Macdonald, I. G. (1995). Symmetric Functions and Hall Polynomials, 2nd ed. Oxford Mathematical Monographs. The Clarendon Press, New York. MR1354144

[49] MCKean, H. P. (1977). - $-\Delta$ plus a bad potential. J. Math. Phys. 18 1277-1279. MR0436826

[50] Minami, N. (2015). Definition and self-adjointness of the stochastic Airy operator. Markov Process. Related Fields 21 695-711. MR3494771

[51] OKounkov, A. (2002). Generating functions for intersection numbers on moduli spaces of curves. Int. Math. Res. Not. IMRN 18 933-957. MR1902297

[52] Pastur, L. and Shcherbina, M. (2003). On the edge universality of the local eigenvalue statistics of matrix models. Zh. Mat. Fiz. Anal. Geom. 10 335-365. MR2012268

[53] Pastur, L. and Shcherbina, M. (2011). Eigenvalue Distribution of Large Random Matrices. Mathematical Surveys and Monographs 171. Amer. Math. Soc., Providence, RI. MR2808038

[54] PÉcHÉ, S. (2009). Universality results for the largest eigenvalues of some sample covariance matrix ensembles. Probab. Theory Related Fields 143 481-516. MR2475670

[55] Prähofer, M. and Spohn, H. (2002). Scale invariance of the PNG droplet and the Airy process. J. Stat. Phys. 108 1071-1106. MR1933446

[56] RAmí REZ, J. A., Rider, B. and ViRÁG, B. (2011). Beta ensembles, stochastic Airy spectrum, and a diffusion. J. Amer. Math. Soc. 24 919-944. MR2813333

[57] Simon, B. (2005). Functional Integration and Quantum Physics, 2nd ed. AMS Chelsea Publishing, Providence, RI. MR2105995

[58] Simon, B. (2005). Trace Ideals and Their Applications, 2nd ed. Mathematical Surveys and Monographs 120. Amer. Math. Soc., Providence, RI. MR2154153

[59] Sodin, S. (2014). Several applications of the moment method in random matrix theory. Preprint. Available at arXiv:1406.3410. 
[60] Sodin, S. (2015). A limit theorem at the spectral edge for corners of time-dependent Wigner matrices. Int. Math. Res. Not. IMRN 17 7575-7607. MR3403994

[61] Soshnikov, A. (1999). Universality at the edge of the spectrum in Wigner random matrices. Comm. Math. Phys. 207 697-733. MR1727234

[62] Sutton, B. D. (2005). The stochastic operator approach to random matrix theory. Ph.D. thesis, Massachusetts Institute of Technology, Cambridge, MA. MR2717319

[63] Tracy, C. A. and Widom, H. (1994). Level-spacing distributions and the Airy kernel. Comm. Math. Phys. 159 151-174. MR1257246

[64] VAlKó, B. and ViRÁG, B. (2009). Continuum limits of random matrices and the Brownian carousel. Invent. Math. 177 463-508. MR2534097

[65] VERSHYNIN, R. (2012). Introduction to the non-asymptotic analysis of random matrices. In Compressed Sensing 210-268. Cambridge Univ. Press, Cambridge. MR2963170

[66] Vervati, W. (1979). A relation between Brownian bridge and Brownian excursion. Ann. Probab. 7 143-149. MR0515820

[67] Widder, D. V. (1941). The Laplace Transform. Princeton Mathematical Series 6. Princeton Univ. Press, Princeton, NJ. MR0005923

[68] Wong, P. (2012). Local semicircle law at the spectral edge for Gaussian $\beta$-ensembles. Comm. Math. Phys. 312 251-263. MR2914063

Department of Mathematics

MassachusetTs Institute of TeChNOLOgY

77 Massachusetts Avenue

CAMBRIDGE, MASSACHUSETTS 02139

USA

AND

INSTITUTE FOR INFORMATION TRANSMISSION PROBLEMS

OF RUSSIAN ACADEMY OF SCIENCES

BOLSHOY KARETNY PER. 19, BUILD.1

MosCOW 127051

RUSSIA

E-MAIL: vadicgor@gmail.com
ORFE DEPARTMENT

PRINCETON UNIVERSITY

202 SHERRERD HALL

PRINCETON, NEW JERSEY 08544

USA

E-MAIL: mshkolni@gmail.com 